\title{
Evolution in sacral neuromodulation for lower urinary tract symptoms
}

Citation for published version (APA):

van Voskuilen, A. C. (2012). Evolution in sacral neuromodulation for lower urinary tract symptoms. [Doctoral Thesis, Maastricht University]. Datawyse / Universitaire Pers Maastricht. https://doi.org/10.26481/dis.20121203av

Document status and date:

Published: 01/01/2012

DOI:

10.26481/dis.20121203av

Document Version:

Publisher's PDF, also known as Version of record

\section{Please check the document version of this publication:}

- A submitted manuscript is the version of the article upon submission and before peer-review. There can be important differences between the submitted version and the official published version of record.

People interested in the research are advised to contact the author for the final version of the publication, or visit the DOI to the publisher's website.

- The final author version and the galley proof are versions of the publication after peer review.

- The final published version features the final layout of the paper including the volume, issue and page numbers.

Link to publication

\footnotetext{
General rights rights.

- You may freely distribute the URL identifying the publication in the public portal. please follow below link for the End User Agreement:

www.umlib.nl/taverne-license

Take down policy

If you believe that this document breaches copyright please contact us at:

repository@maastrichtuniversity.nl

providing details and we will investigate your claim.
}

Copyright and moral rights for the publications made accessible in the public portal are retained by the authors and/or other copyright owners and it is a condition of accessing publications that users recognise and abide by the legal requirements associated with these

- Users may download and print one copy of any publication from the public portal for the purpose of private study or research.

- You may not further distribute the material or use it for any profit-making activity or commercial gain

If the publication is distributed under the terms of Article $25 \mathrm{fa}$ of the Dutch Copyright Act, indicated by the "Taverne" license above, 


\section{Evolution in sacral neuromodulation for Lower Urinary Tract Symptoms}


(c) Copyright Anthonie Cornelis van Voskuilen, Maastricht 2012.

All rights reserved. No part of this thesis may be reproduced of transmitted in any form of by any means, electronic or mechanical, including photocopying, recording, or any information storage or retrieval system without permission from the author, or when appropriate, from the publishers of the publications.

Alle rechten voorbehouden.

Niets uit deze uitgave mag worden verveelvoudigd, opgeslagen in een geautomatiseerd gegevensbestand en/of openbaar gemaakt in enige vorm of op enige wijze, hetzij elektronisch, mechanisch, door fotokopieën, opnamen of op enige andere manier zonder voorafgaande schriftelijke toestemming van de uitgever.

Vormgeving en druk: Datawyse | Universitaire Pers Maastricht

ISBN 9789461591876 


\title{
Evolution in sacral neuromodulation for Lower Urinary Tract Symptoms
}

\author{
PROEFSCHRIFT \\ ter verkrijging van de graad van doctor \\ aan de Universiteit Maastricht, \\ op gezag van de Rector Magnificus, \\ Prof. dr. L.L.G. Soete \\ volgens het besluit van het College van Decanen, \\ in het openbaar te verdedigen op \\ maandag 3 december 2012 \\ om 14:00 uur
}

door

Anthonie Cornelis van Voskuilen

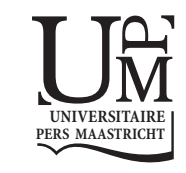




\section{Promotor}

Prof. dr. Ph. E. V. van Kerrebroeck

Prof. dr. R. A. de Bie

\section{Copromotoren}

Dr. E. H. J. Weil

\section{Beoordelingscommissie}

Prof. dr. H.W.M. Steinbusch (voorzitter)

Prof. dr. C.G.M.I. Baeten

Dr. J.P.F.A. Heesakkers (St. Radboud Universiteit, Nijmegen)

Prof. dr. M.A.E. Marcus

Prof. dr. D. de Ridder (Katholieke Universiteit Leuven, België) 


\section{Table of Contents}

Chapter 1 General Introduction and outline of the thesis 7

Chapter 2 Backgrounds of normal voiding and indications for Sacral $\quad 15$

Neuromodulation

Chapter 3 Results of sacral neuromodulation therapy for urinary voiding 31 dysfunction: outcomes of a prospective, worldwide clinical study. J Urol. 2007 Nov;178(5):2029-34

Chapter 4 Long Term Results of Neuromodulation by Sacral Nerve Stimulation for Lower Urinary Tract Symptoms: a Retrospective Single Center Study Eur Urol. 2006 Feb;49(2):366-72

Chapter 5 Medium Term Experience of Sacral Neuromodulation by tined 59 leadimplantation.

BJU Int. 2007 Jan;99(1):107-10

Chapter 6 Is on-demand sacral neuromodulation in patients with $\mathrm{OAB}$ syndrome a feasible therapy regime? Neurourol Urodyn. 2011 Nov;30(8):1493-6

Chapter 7 The Sexual Response in Patients treated With Sacral Neuromodulation for Lower Urinary Tract Symptoms or Fecal Incontinence.

Urol Int. 2012 Apr 26

$\begin{array}{lll}\text { Chapter } 8 & \text { Discussion and Future perspectives } & 95\end{array}$

$\begin{array}{lll}\text { Chapter } 9 & \text { Summary } & 107\end{array}$

$\begin{array}{lll}\text { Chapter } 10 \quad \text { Nederlandse samenvatting } & 113\end{array}$

$\begin{array}{lll}\text { Chapter } 11 \text { Dankwoord } & 121\end{array}$

Lijst van publicaties

Curriculum Vitae 

Chapter 1

General introduction and outline of the thesis 
CHAPTER 1 


\section{GENERAL INTRODUCTION}

The term Lower Urinary Tract Symptoms (LUTS) describes a wide range of conditions regardless to the cause of these symptoms. Both symptoms of overactive bladder, such as urgency urinary incontinence or urgency-frequency, and urinary retention are included in the term LUTS.

Urgency urinary incontinence has a prevalence of $5.6 \%$ in the Netherlands. ${ }^{1}$ For the Dutch population of about 16 million people this means that approximately 896,000 persons are suffering from this ailment. Urinary incontinence and urgencyfrequency have a severe impact on the patients' quality of life. ${ }^{2}$ The expenditure in the United States of America for urinary incontinence in female patients was 452.8 million dollar in the year 2000.

Patients with voiding dysfunctions, such as urinary retention with or without outflow obstruction, make up another group of patients with LUTS. There are no figures published on the occurrence of this condition, but this condition influences the quality of life of the patients and gives considerable associated costs as well, as these patients usually require frequent self-catheterization to empty their bladder. In conclusion, these two groups of LUTS mentioned above form an important problem in health care. Both the loss in quality of life as the high associated costs of disease justify the need for adequate treatment.

In 1990 sacral neuromodulation (SNM) became available as a new treatment option in patients with urgency urinary incontinence, urgency-frequency and later nonobstructive urinary retention. Sacral neuromodulation is modulation of the voiding reflex system by stimulating a sacral nerve with electrical current. Details on technique and mechanism of action are described further on in this thesis.

In the last two decades sacral neuromodulation obtained a place in the list of therapy options a physician has to offer to patients with LUTS, resistant to conservative therapy.

\section{AIMS OF THIS THESIS}

In this thesis long term results of SNM with regards to efficacy and safety, will be discussed. This thesis presents the results from both a multicenter as a single center study on long term results. It also contains research on an improved implant method and data on a new type of stimulator settings.

Chapter 1 contains this introduction and outlines the aims of this thesis. In addition it contains an overview on the history and procedure of SNM. Chapter 2 gives an 
overview of the neurophysiology of normal voiding. In addition, the current knowledge and hypotheses on the mechanism of action of SNM are discussed and a summary of the pathophysiology of the current indications of SNM is given.

The data of the prospective multicenter study into the five-years results of sacral neuromodulation became available and are presented in Chapter 3. This study was carried out to measure long term efficacy and safety of SNM. It is an extension of the initial study by the manufacturer of the SNM equipment, Medtronic Inc, to gain FDA-approcal for SNM.

In addition, a retrospective study was carried out into the results of sacral neuromodulation in the department of Urology in the Academisch Ziekenhuis in Maastricht. (Chapter 4). This study shows the influence of technical developments on the outcome of SNM and the resolution of adverse events encountered when applying sacral neuromodulation.

One of these developments was the introduction of a minimal invasive method of electrode implant in 2003. Initial results with this method in our clinic are described in Chapter 5, as this method changes the SNM treatment algorithm.

Chapter $\mathbf{6}$ is a study into the feasibility of interruption of neuromodulation. Intermittent neuromodulation might have a few advantages. It saves battery life of the implanted pulse generator and it might postpone or prevent the loss of efficacy in long term that some patients experience.

In our outpatient clinic there was a number of female patients with an implanted neuromodulation system who spontaneously reported improvement in their sexual functioning. This led to the questions whether this improvement could be objectified and if this improvement was due to a physiological reaction on stimulation of the sacral root, leading to direct genital stimulation, or due to a psychological reaction because of an improved well-being through symptom resolution. The resulting study is described in Chapter 7 .

Chapters 8 and 9 conclude this thesis and contain an overview of future perspectives in SNM

\section{SACRAL NEUROMODULATION}

\section{History}

The use of electricity for therapeutic goals was already described by Scribonius Largus in $46 \mathrm{AD}$. He described the use of electrically charged eels to treat pain in ancient Rome. In 1780 Galvani first observed the twitching of muscles in a frog's leg when touched with an electrically charged object. In the following centuries, technical improvements and better understanding of electricity and biology led to the advent of Functional Electrical Stimulation (FES) as a therapeutic tool. 
Already in the $20^{\text {th }}$ century urinary retention was treated by direct electrical stimulation of the detrusor or by transurethral stimulation. ${ }^{3}$ At the end of the sixth decade the application of FES became technically easier due to the development of the transistor. The interest for electrotherapy for lower urinary tract symptoms grew. ${ }^{4}$ FES was used for restoring micturition in patients with retention and in patients with incontinence. ${ }^{5,6}$

In 1974 Brindley published on the implantation of electrodes on the sacral nerve roots to stimulate bladder afferents. ${ }^{7}$ In 1976 this method of bladder stimulation was first applied in paraplegic patients to achieve bladder emptying.

In implanted patients the ventral roots of S2, S3 and S4 are stimulated to achieve bladder contraction. A dorsal rhizotomy was carried out in these patients to abolish detrusor-sphincter dyssynergia and to decrease incontinence at higher bladder volumes. As the sphincter is stimulated simultaneously, voiding takes place due to interruption of the stimulation. The smooth detrusor muscle relaxes slowly, while the striated sphincter relaxes more rapidly, allowing for evacuation of urine between consecutive stimuli. This phenomenon is known as post stimulus voiding.

The results of Brindley in paraplegic patients led to research into methods for selective stimulation of nerves to the bladder. In experiments using horseradish peroxidase staining Thuroff discovered that the somatic innervation of the sphincter was represented in the spinal cord in the pudendal nucleus and the autonomic innervation of the bladder was controlled through a parasympathic nucleus. ${ }^{8}$

Selective stimulation of these nuclei at spinal cord level is not possible, due to the localization of these nuclei in the spinal cord. In the eighties a group of researchers in Los Angeles did extensive research into the optimal site and method of bladder stimulation in spinalized dogs and later in patients with neurogenic bladder dysfunction. ${ }^{9}$ They found that selective stimulation of sacral roots was feasible and led to suppression of overactive bladder contractions. An explanation of this phenomenon was that the sphincter was stimulated by stimulation of efferent nerve roots and led to activation of the continence reflex.

It was also hypothesized that stimulation of the sphincter complex could prevent incontinence by increasing outflow resistance. However, when research methods were more refined, it became clear that the reduction in incontinence was caused by inhibition of the detrusor muscle, instead of a direct effect on the sphincter. ${ }^{10}$ This effect was confirmed by other authors who used different methods of electrical stimulation, among others intravaginal stimulation and bulbocavernous stimulation. $^{11}$

In the following period, Tanagho and Schmidt refined their stimulation techniques and developed the sacral neurostimulation method using foramen S3. ${ }^{12,13}$ In 1997 SNM achieved FDA approval. The indication of this therapy was later on extended to patients with idiopathic urgency symptoms and urinary retention. ${ }^{14}$ 
Sacral Nerve Stimulation (SNS) therapy, as devised by Tanago and Schmidt, made use of a quadripolar lead connected to an Implantable Pulse Generator (IPG, colloquially known as 'bladder pacemaker'). These were implanted in a surgical procedure under general anesthesia. The lead was sutured over the sacral periost and subcutaneously tunneled with an extension wire to the IPG that was implanted in a subcutaneous pocket at the left or right lower abdominal quadrant.

\section{PROCEDURE OF SNM}

\section{Temporary test procedure}

Patients that are candidates for SNM first undergo a test procedure, the so-called Peripheral Nerve Evaluation (PNE) During this test both acute and subchronic resonses to electrical stimulation are evaluated.

After positioning the patient in a prone position, both third sacral foramina are identified, based on the superior illiac spines (at S2 level), the tip of the coccyx and the inferior illiac spine (at the level of S3).

Both S3 foramina are located one centimetre laterally from the midline. ${ }^{15}$ After local anesthesia, taking care not to infiltrate the nerve, a needle is introduced. When the needle is in position, a small electric current is applied and slowly increased until the patient has a tingling, pulling or tapping sensation at the perineum or, respectively in females or males, the labia or scrotum.

Expected motor responses at correct electrode placement are contraction of perianal muscles, the so-called 'bellows response' and plantar flexion of the great toe. When the responses are indicative of correct needle placement, a thin temporary lead is introduced through the needle and the needle is withdrawn, taking care to leave the lead in place. After careful dressing, an external stimulator is connected and the patient is sent home with instructions how to handle the stimulator and to avoid sudden flexion and extension of the back and carrying heavy objects. The electrode position is checked with an X-ray of the pelvis in two directions.

After about week the electrode is removed. The patient has kept voiding diaries before and during stimulation. The voiding diary contains number of voids/day, volume/void, changes of incontinence pads/day, number of catheterizations, catheterized volume/catheterization. The diary data before and during stimulation are compared by computer software. A candidate is eligible for definitive electrode implant when there is at least $50 \%$ improvement in relevant voiding parameters during the test stimulation period. 


\section{Definitive implant}

Before the development of a minimal invasive implant method, implant took place in the operating room under general anesthesia.

Nowadays definitive implants are performed under local anesthesia. ${ }^{16}$ The procedure starts in the same manner as the PNE, but when the responses are satisfactorily, a guide wire is advanced through the stimulation needle. After removing the needle a stylet is introduced. An quadripolar lead is placed through the stylet and its depth and is checked with fluoroscopy. Silicone tines allow for lead fixation once the stylet is removed.

The lead is connected to an extension wire and tunneled subcutaneously to a created pocket about 3 centimetres below the illiac crest. An Implantable Pulse Generator (IPG) is connected to the extension wire and placed in the pocket. After wound closure and dressing, the IPG is programmed. Programming takes place with a telemetry device. As the lead has four poles, lead configuration is based on the best sensory response. Initial stimulation parameters are: continuous stimulation with a frequency of $10 \mathrm{~Hz}$ and pulsewith of $210 \mu \mathrm{sec}$. The amplitude is set to such a voltage that the patient feels the stimulation, but not in an unpleasant manner. Figure 1 is a schematic representation of an implanted SNM system.

The patient is supplied with a Patient Programmer, that enables him/her to increase or decrease stimulation amplitude and to switch the stimulator off and on.

After implant, regular follow-up visits are planned to check whether system settings are still optimal. Furthermore a troubleshooting service is available for patients with problems with their SNM system.

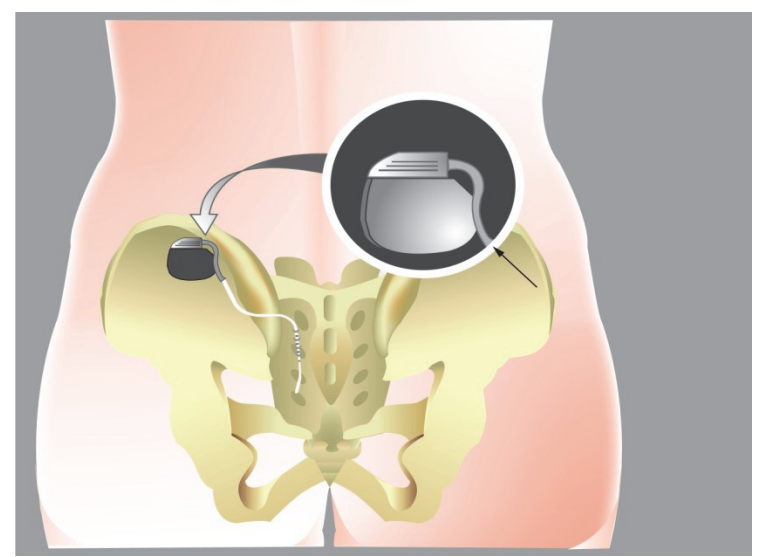

Figure 1. Schematic representation of an implanted SNM system. The electrode is positioned in the left third sacral foramen and connected to an Implantable Pulse Generator that is implanted in the upper left buttock. 


\section{REFERENCES}

1. C.H. van der Vaart, De Invloed Van Urine-incontinentie Op De Kwaliteit Van Leven Bij Thuiswonende Nederlandse Vrouwen Van 45-70 Jaar, Ned Tijdschr Geneeskd, 2000.

2. J.M. van Geelen, Urogenital Symptoms and Resulting Discomfort in Non-institutionalized Dutch Women Aged 50-75 Years. Int-Urogynecol-J-Pelvic-Floor-Dysfunct, 2000.

3. T. Tanagho Fandel, Neuromodulation in Voiding Dysfunction: A Historical Overview of Neurostimulation and Its Application, Urologic Clinics of North America 32 (2005): 1-10.

4. F Katona and H B Eckstein, Treatment of Neuropathic Bladder by Transurethral Electrical Stimulation, Lancet 1, no. 7861 (April 27, 1974): 780-781.

5. D Q Hill and C A Griffiths, An Electronic Stimulator for the Treatment of Urinary Incontinence, British Journal of Urology 40, no. 2 (April 1968): 187-190.

6. S Alexander and D Rowan, Electrical Control of Urinary Incontinence by Radio Implant. A Report of 14 Patients. The British Journal of Surgery 55, no. 5 (May 1968): 358-364.

7. Brindley GS, Emptying the Bladder by Stimulating Sacral Ventral Roots. J Physiol 237, no. 2 (1974): 15P-16P.

8. J W Thüroff et al., Regional Topography of Spinal Cord Neurons Innervating Pelvic Floor Muscles and Bladder Neck in the Dog: a Study by Combined Horseradish Peroxidase Histochemistry and Autoradiography. Urologia Internationalis 37, no. 2 (1982): 110-120.

9. Schmidt R Tanagho EA, Electrical Stimulation in the Clinical Management of the Neurogenic Bladder. J Urol 140 (1988): 1331-1339.

10. M Fall and S Lindström, Electrical Stimulation. A Physiologic Approach to the Treatment of Urinary Incontinence. The Urologic Clinics of North America 18, no. 2 (May 1991): 393-407.

11. Di Benedetto M Yalla SV, Urethral Striated Sphincter Responses to Electro-bulbocavernosus Stimulation. J Urol 119, no. 3 (1978): 406-409.

12. Senn E Schmidt RA, Functional Evaluation of Sacral Nerve Root Integrity. Report of a Technique. Urology 35, no. 5 (1990): 388-92.

13. E. A. Tanagho, Concepts of Neuromodulation, Neurourol Urodyn 12, no. 5 (1993): 487-8.

14. H. S. Shaker and M. Hassouna, Sacral Root Neuromodulation in Idiopathic Nonobstructive Chronic Urinary Retention. J Urol 159, no. 5 (1998): 1476-8.

15. S. T. Hasan et al., Transcutaneous Electrical Nerve Stimulation and Temporary S3 Neuromodulation in Idiopathic Detrusor Instability. J Urol 155, no. 6 (1996): 2005-11.

16. M. Spinelli et al., New Percutaneous Technique of Sacral Nerve Stimulation Has High Initial Success Rate: Preliminary Results. Eur Urol 43, no. 1 (2003): 70-4. 
Chapter 2

Backgrounds of normal voiding and indications for Sacral Neuromodulation 
CHAPTER 2 


\section{ANATOMY AND NEURAL CONTROL OF VOIDING}

The urinary tract consists of the kidneys, ureters, the bladder and the urethra with its sphincter system. In the male the prostate is considered a part of the urinary tract as well. For clinical reference the urinary tract is divided in two parts. The upper urinary tract consists of the kidneys with the abdominal part of the ureters. The lower urinary tract is formed by the part of the ureters that is below the level of the iliac vessels, the bladder and the urethra. The function of the urinary tract is to collect and store urine and dispose of it at a convenient moment.

\section{Components of the Urinary System}

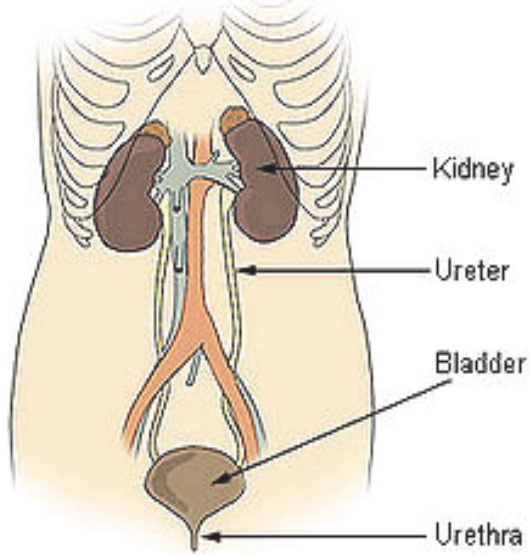

Figure 1. A schematic diagram of the urinary tract.

The bladder is a hollow muscular organ. Its average capacity for storing urine is $\mathbf{4 0 0}$ $-500 \mathrm{ml}$. The inner surface of the bladder is covered with transitional epithelium, which consists of six layers of cells. In an empty bladder it is folded, but when the bladder is full, it becomes a smooth surface. Under this layer is the lamina propria, a layer of fibro-elastic connective tissue that supports distension of the bladder.

External to the lamina propria is the detrusor muscle, a smooth muscle with fiber bundles running over the bladder. At the level of the bladder neck the detrusor forms three clearly distinguishable layers, at the top of the bladder the separate layers cannot be identified. The inner and outer layers are circularly oriented, while the middle layer has a longitudinal orientation. This arrangement of the detrusor muscle allows for efficient emptying of the bladder.

In the storage phase unwanted urine outflow from the bladder is prevented by two sphincter systems. The internal sphincter lies at the bladder neck and consists of 
smooth muscles. It works without conscious control and is controlled by sympathetic innervation. ${ }^{1}$

The external sphincter contains striated muscles and is under voluntary control. The axons that control the external sphincter are localized in the nucleus of Onuf, located at S1 and S1 level in the anterior horn of the spinal cord.

Voiding is controlled by three subsystems of the nervous system: the parasympathetic, the orthosympathetic and the voluntary or somatic system.

The parasympathetic nerves of the bladder and the sphincter originate in the sacral parasympathetic nucleus (SPN) at the second to fourth sacral segment and innervate the pelvic plexus. Their postganglionic fibers innervate the bladder, the internal sphincter and the urethra. The main neurotransmitter of the parasympathetic system is acetylcholine. The function of the parasympathetic innervation of the lower urinary tract is to initiate and propagate voiding.

The orthosympathetic hypogastric nerves travel from the hypogastric plexus to the bladder neck and proximal urethra and innervate the muscle cells that form the internal sphincter. These nerves play an important part in the storage phase by relaxing the bladder, contracting the bladder neck and closure of the urethra and therefore facilitating urine storage.

The voluntary innervation of the lower urinary tract carries information from the nucleus of Onuf. The Onuf nucleus lies in the ventral horn of the sacral spinal cord. The A $\alpha$ motor neurons that originate from the Onuf nucleus towards the external urethral sphincter are part of the pudendal nerve.

The afferent innervation of the bladder runs through the pudendal nerve, the pelvic nerve and the hypogastric nerve. It consists of myelinated A $\delta$-fibers that carry information from smooth muscle cells about bladder filling and unmyelinated Cfibers that respond to bladder wall stretch, overdistention and noxious stimuli. Most C-fibers are silent and are only recruited when there is a painful stimulus.

During the storage phase the bladder is filled with urine from the kidneys. Because of the elasticity of the bladder wall the pressure in the bladder rises almost unnoticeably. Assumed is that there is some uncoordinated activity in the smooth muscle cells of the bladder during filling, but it is not synchronous and does not lead to increased pressure.

Information about bladder fullness is carried from the smooth detrusor cells through $A \delta$-fibers to the spinal cord. In the posterior horn of the spinal cord the $A \delta$ fibers activate interneurons that stimulate both the external sphincter through the pudendal nerve and the orthosymphatic system.

During filling the sympathetic system controls the bladder, giving relaxation of the detrusor muscle and contraction of the internal sphincter. The parasympathetic system is inhibited. 
The information of the A $\delta$-fibers is carried to the cerebrum through Lissauer's tract up to the periaqueductal grey (PAG). The PAG is said to be involved in processing visceral afferent information and relaying it to other cerebral structures. The PAG is also considered as a part of the emotional system and plays a role in the processing of fear and anxiety. ${ }^{2}$ Therefore the PAG receives information from many other cerebral structures, including the frontal cortex and from parts of the limbic system. $^{3}$ The PAG has a projection to the pontine micturition center (PMC), which is clinically referred to as the nucleus of Barrington. ${ }^{4,5,6}$

The PMC consists of two separate regions. The L-region lies laterally in the PMC and is clinically referred to as 'the continence center'. It projects to Onuf's nucleus and facilitates contraction of the external sphincter. Stimulation of the L-region results in an increase of plevic floor EMG in cats, while a lesion in this area gives

incontinence. $^{7}$

The M-region of the PMC lies medially in the pons and is colloquially known as the 'micturition center'. Electrical stimulation of the M-region of the PMC results in relaxation of the pelvic floor, followed by bladder contraction. Bilateral lesion of the $M$ region results in urinary retention. ${ }^{8}$

In essence, storing urine and voiding is executed by a series of bulbospinal reflex arcs, controlled by cerebral structures. The filling sensations of the bladder are carried through the $A \delta$-fibers to the periaqueductal gray and relayed to the Barrington nucleus in the pons. In the storage phase of the micturition cycle the Onuf nucleus is stimulated by the L-region in the Barrington nucleus. The external sphincter is contracted and the detrusor is relaxed, allowing for storage of urine.

Micturition usually begins voluntarily in healthy adult subjects, when time and place are acceptable for voiding. Although we know that voiding is started by voluntarily switching on the micturition reflex, it is not known which structure is responsible for switching. As the Barrington nucleus controls both continence and voiding in respectively the L-region and M-region, it would be logical to assume that theBarringon nucleus regulates initiation of voiding. However, fiber tracing studies in cats show that in the pons are no interconnections between L- region and M-region. ${ }^{9}$ Therefore, other cerebral structures must be responsible for the initiation of the voiding phase.

Research with functional neuroradiology techniques as PET, fRMI or SPECT has implicated many different cerebral structures in the start of the voiding phase, such as the prefrontal cortex, insula, anterior cingulate gyrus and cerebellum. ${ }^{10,11}$ 
Stimulation of the M-area of the PMC gives a sharp decrease of the urethral pressure and relaxation of the pelvic floor, followed after a few seconds by contraction of the bladder. The sympathetic and voluntary spinal guarding reflexes that are responsible for continence are inhibited by the M-area. Voiding is sustained by a positive-feedback mechanism. Contraction of the detrusor muscle increases bladder wall stress. The $A \delta$-fibers increase their firing rate to the PMC, thereby increasing parasymphatic neuronal command to the detrusor. Another positive-feedback mechanism is that when urine passes the urethra, a reflex arc through the PMC induces bladder contraction.

Voiding stops when the bladder is empty or contains a minimal amount of urine. The pressure in the bladder decreases and the reflexes that prolong the micturition switch off.

\section{MECHANISM OF ACTION OF SNM}

The exact way how SNM works in urinary symptoms is not clear.

An initially suggested hypothesis was that neurostimulation on the S3 level amplified the continence reflex by stimulation of the pelvic nerve, running to the external sphincter. ${ }^{12}$ The contraction of perianal muscles, observed in acute stimulation of the S3 nerve root, was proposed as support for this theory.

This hypothesis was disproved by Fowler et al, who found increased latencies with electromyography of the anal sphincter on stimulation of the S3 root, compared to stimulation of the pudendal nerve. These findings suggest that SNM acts through a polysynaptic reflex, but this study gave no distinction between a spinal or a supraspinal reflex.$^{13}$

In 2002, Schurch et al performed neuromodulation in patients with complete spinal cord injury. ${ }^{14}$ Through latency measurements the involvement of afferent pathways and spinal reflexes were confirmed. But as the SNM did not improve voiding in these patients with complete spinal cord injury, it was suggested that there are also supraspinal mechanisms involved in the action of SNM.

In 2002 Braun at al described their results of electroencephalography in 10 patients with SNM. ${ }^{15}$ Four patients had neurogenic LUTS, 6 patients had idiopathic LUTS. They found a cortical potential complex at the postcentral gyrus in all patients, regardless if patients felt the simulation or not. The postcentral gyrus is the primary somatosensory cortex, so the occurrence of a potential when using SNM would only be logical in patients who feel the stimulation.

In addition, Positron Emission Tomography (PET) studies have been performed in patients with urinary retention due to the Fowler Syndrome and compared with healthy control subjects. ${ }^{16}$ The 8 included patients with Fowler syndrome did not 
show activity in the brain stem on bladder fullness, while the control subjects did. With an active SNM system there was activity in the brainstem in the patients and decrease of activity in the cingulate cortex, suggesting a cerebral contribution of SNM in patient with Fowlers' syndrome.

A study by the same research group with $\mathrm{fMRI}$ in patients with Fowler's syndrome showed a different response in the periaqueductal gray on bladder filling when the SNM system is on. ${ }^{17}$ With a near empty bladder the PAG is not active in healthy patients. In patients with Fowler's syndrome the PAG is active even when there is a little bladder filling. With SNM the activation of the PAG seems to normalize. The authors hypothesize that SNM blocks inhibition of afferent information from the bladder.

In 2006 PET scanning was also performed in 20 patients with SNM for overactive bladder symptoms. ${ }^{18}$ The researchers found decreases in activation in the cingulate gyrus, in the thalamus and in the ventromedial frontal cortex. In acutely stimulated patients, activation was found in the postcentral gyrus, the insula and the ventromedial frontal cortex. Therefore they confirmed the results of Braun et al and indicated a similar mechanism in $O A B$ as Dasgupta et al found in Fowler's syndrome.

Furthermore, in animal experiments with a neurogenic bladder model Shaker et al found inhibition of reflex contractions of the bladder with neuromodulation, together with changes in neuropeptides levels that suggest a mechanism involving a decrease in activation of the C-afferent nerve fibers. ${ }^{19}$ Normally, the C-afferent fibers are silent. The increased expression of neuropeptides point to increased activation in spinal cord injury. It is, however, not clear whether these results are valid as well for idiopathic $O A B$ and retention.

In conclusion, based on the current information on the working mechanism of SNM, SNM might act mostly on the processing of sensory information from the bladder. There are indications that, at least in urgency-related symptoms, SNM has an influence on pathological transmission of afferent information from the bladder through the C-fibers, based on the results of Shaker et al. Furthermore, both in OAB and in retention there are indications that the processing of afferent information in the PAG and in the cingulate cortex is normalized with SNM. 


\section{INDICATIONS FOR SNM}

\section{Symptoms of idiopathic overactive bladder}

Overactive bladder (OAB) is defined by the Incontinence Society (ICS) in 2002 as urgency, with or without urge incontinence, and usually with frequency and nocturia. ${ }^{20}$ The term $O A B$ is a description of a symptom complex without information on the cause of the symptoms. The most characteristic symptom in this complex is urgency. The ICS definition of urgency is: "a sudden compelling desire to pass urine that is difficult to defer." Urgency is considered the driving force behind the other symptoms that make out the ICS definition of $O A B .{ }^{21} O A B$, with or without urinary incontinence, has a severe impact on quality of life, as patients with 'OAB wet' (OAB with incontinence) usually become dependent on sanitary pad use and patients with 'OAB dry' (OAB without incontinence) tend to plan their activities based on the vicinity of a toilet.

Research into the causes of $O A B$ is still ongoing. There are several contributing factors. There is strong evidence for a disbalance in voiding reflexes due to changes in the afferent innervation from the bladder. This hypothesis is among others based on the efficacy of intravesical administration of capsaicin or resiniferatoxin for symptoms of overactive bladder. Capsaicin is assumed to desensitize the C-afferent fibers running from the bladder. ${ }^{22,23,24}$ Recently, a relation is found between autonomic dysfunction and $O A B$, suggesting a dysbalance in the voiding reflex. ${ }^{25}$

Other research is focused on the properties and signal transduction between the myocytes that form the detrusor. Brading showed in 1997 that detrusor myocytes change their properties after denervation. ${ }^{26}$

Recent neuroimaging studies also imply a cerebral mechanism in the pathogenesis of OAB symptoms, but more research is needed. ${ }^{27}$

The diagnostic work-up of $O A B$ is mainly aimed towards exclusion of possible known causes of $O A B$, such as neurological disease or infravesical obstruction. The value of cystometry for diagnosing idiopathic $O A B$ is still under debate, as only 50 $80 \%$ of patients with $O A B$ has evidence of an overactive detrusor on urodynamic investigation. $^{28,29}$ It has its value, however, in excluding other causes of LUTS.

The treatment algorithm in OAB starts with lifestyle counseling, such as decrease of fluid/caffeine intake and weight loss. This can be supplemented by pelvic floor training of pelvic floor myofeedback. A pharmacological option is the use of antimuscarinic medication. These drugs antagonize the acetylcholine receptor and there are several preparations that act more or less specifically on the receptors on the bladder wall.

However, in a considerable proportion of patients relief of their OAB symptoms is not satisfactorily or the antimuscarinic drugs are not well tolerated because of their side-effects. ${ }^{30}$ These side-effects include: a dry mouth, dizziness or visual distur- 
bances. Estimated discontinuation rates of antimuscarinic drugs for $O A B$ are $42 \%$ after four months and $72 \%$ after one year. ${ }^{31}$

Second line therapy of $O A B$ consists of botulin toxin application in the bladder or functional electrical stimulation.

Botulin neurotoxin (BTX) is a neurotoxin, produced by the bacteria Clostridium botulinum. The protein acts on the terminal ends of axons and prevents the release of neurotransmitter. It has a preference for inhibiting Acetylcholine release and is therefore used as a local muscle relaxant. Medical uses for BTX include: reduction of muscle spasm or dystonia or treatment of excessive sweating. Its cosmetic use as treatment for facial wrinkles is also widely known.

In urology, BTX is injected intravesically in the detrusor. BTX has been used in patients with neurogenic overactive bladder. ${ }^{32}$ Since 2005 it is applied in patients with idiopathic overactive bladder as well. ${ }^{33,34}$ Depending on study methodology the effectiveness of intravesical BTX ranges between $40-80 \% .{ }^{35,36}$ Side-effects of intravesical BTX are: urinary retention requiring periodic intermittent catherization, urinary tract infections or extravesical effects of the BTX. The effects of BTX wear off, therefore patients need repeated injection procedures with an interval of 4-9 months.

The use of BTX is not FDA-approved yet, due to the lack of data on long term effects.

The other second line treatment is neuromodulation by electrostimulation.

A site for neuromodulation is the posterior tibial nerve. ${ }^{37}$ Percutaneous Tibial Nerve stimulation has as an advantage over SNM that it is less invasive, but patients need repeated visits to the outpatient clinic to maintain efficacy. ${ }^{38}$

In 2000 a multicentre trail on sacral neuromodulation in 51 patients with $O A B$ symtoms showed significant symptom relief compared to a control group. After turnig the stimulator off the symtoms recurred, but after turning the stimulator on, the symptoms improved until up to 24 months. ${ }^{39}$ Further long term results make up a part of this thesis.

Before the introduction of functional electrical stimulation and BTX the only therapeutic options for severe $O A B$ symptoms that did not respond to lifestyle advise or drugs were invasive surgical procedures, such as bladder augmentation or urinary deviation procedures.

\section{Non-obstructive urinary retention}

Urinary retention is the loss of ability to urinate. The most likely cause of urinary retention is infravesical obstruction by an enlarged prostrate due to benign prostatic hyperplasia in males or urethral stenosis. 
Functional causes of urinary retention are decreased or absent tonus of the detrusor muscle or hypertonia of the sphincter in pelvic floor dysfunction.

An example of urinary retention in pelvic floor dysfunction is the Fowler syndrome, consisting of urinary retention with abnormal electromyographic activity of the external urethral sphincter and polycystic ovaries. ${ }^{40}$ The voiding dysfunction in this syndrome tends to react favourably on SNM. ${ }^{41}$

Idiopathic urinary retention can occur after surgical procedures, especially after pelvic bowel surgery or surgery for spinal disc herniation. ${ }^{42}$ In many patients, however, a cause for the retention can not be found.

Patients with functional urinary retention rely on clean intermittent catheterization (CIC) for emptying their bladder. Having to catheterize several times per day is an inconvenience. Little is known in the literature on the occurrence of urinary tract infections in patients who catheterize because of idiopathic urinary retention as most research on this matter is carried out on children or in patients with retention on a neurological basis.

In 2001 a randomized trail of SNM in patients with idiopathic urinary retention was published. ${ }^{43}$ The researchers found that $69 \%$ of the 37 patients in the SNM group were able to void completely without catheterization and in patients who kept catheterizing significant reduction of volume per catheterization was found.

\section{Neurogenic bladder dysfunction}

The symptomatology of neurogenic bladder dysfunction depends on the neurological localization of the lesion.

The most important distinction is the central (upper motor neuron) or peripheral (lower motor neuron) localization of the lesion.

Central lesions occur in cerebro or in the spinal cord, while the peripheral nerve system consists of the nerves that run from the spinal cord, including the cauda equina, to the bladder and sphincter complex. The localization of the lesion depends for a part on the neurological disease.

Peripheral lesions give a detrusor hypotonia, that can be combined with an hypotonic sphincter. The clinical picture is either urinary retention or overflow incontinence. Central lesions give different clinical signs, depending on the localization of the lesions. Hyperreflexia of the detrusor, with or without detrusorsphincter dissynergia, is seen in spinal cord lesions at T12 or above. In the acute stage, the bladder is flaccid due to the spinal shock phase, lasting up to several months. Below T12 the spinal nerves branch out to their target organs. Therefore, a lesion below T12 is a lower motor neuron lesion and gives detrusor hypotonia.

When SNM was introduced, neurogenic voiding symtoms formed a contraindication for SNM, as it was believed that an intact supraspinal reflex arc is re- 
quired for succesful SNM. ${ }^{44,45}$ Furthermore, many neurological conditions are progressive and therefore an initially favourable effect of SNM can wear off as the neurologic disease progresses.

A multicenter long term SNM study in nine patients with neurogenic OAB due to spinal cord injury all showed clinical improvement. ${ }^{46}$ But another study on temporary SNM in three patients with complete spinal cord lesions showed anal responses on EMG, but no results on voiding complaints. ${ }^{47} \mathrm{~A}$ reason for the discrepancy in these two studies might be that the completeness of the spinal cord lesion was not mentioned in the multicenter study.

Chaabane et al published long term results of SNM in a group of patients with neurogenic $O A B$ or urinary retention. ${ }^{48}$ Of the 62 patients tested with a temporary implant, 37 had a definitive SNM implant. 27 Patients had complete improvement after a mean follow-up of $4.3 \pm 3,7$ year.

In a study on SNM in patients with $\mathrm{OAB}$ or retention after incomplete spinal cord injury, 9 of 13 patients with neurogenic retention achieved at least 50\% improvement in voiding parameters, in the OAB group 7 of 9 patients had improvement. ${ }^{49}$ The spinal cord lesions were classified as AIS C of D, implying that the patients kept sensibility and at least some muscle activity below their lesion level, and therefore possibly at least partially intact voiding pathways. SNM may be a promising therapy in patients with neurogenic bladder dysfunction, but its success depends on careful selection of potential candidates.

\section{Fecal incontinence and constipation}

From the onset of SNM there were anecdotal stories of patients who had defecation complaints, beside their lower urinary tract symptoms and reported improvement of these symptoms as well.

In 1995 SNM was successfully applied in patients with idiopathic fecal incontinence. ${ }^{50}$ The exact mechanism of action of SNM in fecal complaints is unclear, but in a study on perception of rectal sensation the stimulation seems to alter the rectal sensibility. $^{51}$

In a multicenter study in 2004 on SNM in 37 patients with fecal incontinence, mean number of incontinence episodes per week dropped from 16.4 to 2.0 at 24 months. Subjectively, the ability to postpone defecation improved as well. ${ }^{52}$ Later on, the indication of SNM was expanded to patients with constipation as well. In 2010 a multicenter study was published, analyzing the results of 62 patients. 45 patients achieved at least $50 \%$ symptom reduction during the PNE. At follow-up an average increase of defecation episodes was seen from 2.3 to 6.6 episodes in 39 patients. ${ }^{53}$ Toileting time decreased with almost $50 \%$ from on average 105. minutes to 5.7 minutes. These results led to an increased utilization of SNM in fecal symptoms. 


\section{Pelvic pain}

There are several chronic pelvic pain syndromes described in the literature.

Interstitial cystitis (IC) is a condition characterized by bladder pain, usually relieved by voiding, and frequency, nocturia and pelvic pain. It is a very bothersome condition and it predominantly occurs in women. IC has a multifactorial pathogenesis. Damage to the bladder wall, increased expression of cytokines and changes in neural plasticity are some of the mechanisms that are implicated. ${ }^{54,55}$

As SNM was incidentally applied in patients with refractory complaints of pelvic pain, several researchers started to apply SNM in patients with IC.

In 2001 Maher et al applied temporary SNM in fifteen patients with interstitial cystitis, refractory to other treatment modalities, and found significant increases in volume per void ( 90 to $143 \mathrm{ml}$ ). Number of voids were decreased from 11 to 6 times per day. ${ }^{56}$

The effect of SNM on interstitial cystitis was confirmed by Comiter who performed definitive SNM implants in 17 patients. $^{57}$ After an average follow-up of 14 months the average frequency at daytime decreased from 17.1 to 8.7 and nocturia decreased from 4.5 to 1.1. Mean volume per void increased from 111 to $264 \mathrm{ml}$. ( $p<0.01$ ). Pain scores (from 0 to 10 ) decreased from 5.8 to 1.6 points.

The long term effect of SNM on interstitial cystitis is not clear. Until now there are no results of prospective long-term studies available.

\section{REFERENCES}

1. M. B. Chancellor, Voiding Function and Dysfunction. in Campbell's Urology, vol. 8 (Philadelphia: Saunders, n.d.).

2. M M Behbehani, Functional Characteristics of the Midbrain Periaqueductal Gray. Progress in Neurobiology 46, no. 6 (August 1995): 575-605.

3. Gert Holstege, The Emotional Motor System and Micturition Control. Neurourology and Urodynamics 29, no. 1 (2010): 42-48.

4. F.J.F. Barrington, The Relation of the Hindbrain to Micturition. Brain 44 (1921).

5. F.J.F. Barrington, The Component Reflexes of Micturition in the Cat: I And II. Brain 54 (1931): 177.

6. B F Blok and G Holstege, Direct Projections from the Periaqueductal Gray to the Pontine Micturition Center (M-region). An Anterograde and Retrograde Tracing Study in the Cat. Neuroscience Letters 166, no. 1 (January 17, 1994): 93-96.

7. G Holstege et al., Anatomical and Physiological Observations on Supraspinal Control of Bladder and Urethral Sphincter Muscles in the Cat. The Journal of Comparative Neurology 250, no. 4 (August 22, 1986): 449-461.

8. Derek J Griffiths, The Pontine Micturition Centres. Scandinavian Journal of Urology and Nephrology. Supplementum, no. 210 (2002): 21-26.

9. B F Blok and G Holstege, Two Pontine Micturition Centers in the Cat Are Not Interconnected Directly: Implications for the Central Organization of Micturition. The Journal of Comparative Neurology 403, no. 2 (January 11, 1999): 209-218.

10. S Nour et al., Cerebral Activation During Micturition in Normal Men. Brain: A Journal of Neurology 123 ( Pt 4) (April 2000): 781-789. 
11. Changfeng Tai et al., Brain Switch for Reflex Micturition Control Detected by FMRI in Rats. Journal of Neurophysiology 102, no. 5 (November 2009): 2719-2730.

12. Fall $M$ Lindstrom $S$, The Neurofysiolgical Basis of Bladder Inhibition in Response to Intravaginal Electrical Stimulation. J Urol 129 (1983): 405-410.

13. C. J. Fowler et al., Studies of the Latency of Pelvic Floor Contraction During Peripheral Nerve Evaluation Show That the Muscle Response Is Reflexly Mediated. J Urol 163, no. 3 (2000): 881-3.

14. Schurch et al., Electrophysiological Recordings During the Peripheral Nerve Evaluation (PNE) Test in Complete Spinal Cord Injury Patients. World J Urol. 2003 May;20(6):319-22

15. P M Braun et al., Alterations of Cortical Electrical Activity in Patients with Sacral Neuromodulator. European Urology 41, no. 5 (May 2002): 562-566; discussion 566-567.

16. Ranan Dasgupta et al., Changes in Brain Activity Following Sacral Neuromodulation for Urinary Retention. The Journal of Urology 174, no. 6 (December 2005): 2268-2272.

17. Rajesh Kavia et al., A Functional Magnetic Resonance Imaging Study of the Effect of Sacral Neuromodulation on Brain Responses in Women with Fowler's Syndrome. BJU International 105, no. 3 (February 2010): 366-372.

18. Bertil F M Blok et al., Different Brain Effects During Chronic and Acute Sacral Neuromodulation in Urge Incontinent Patients with Implanted Neurostimulators. BJU International 98, no. 6 (December 2006): 1238-1243.

19. H. Shaker, Role of C-afferent Fibres in the Mechanism of Action in Chronic Spinal Cord Injury. BJU International 85 (2000): 905-910.

20. P. Abrams et al., The Standardisation of Terminology of Lower Urinary Tract Function: Report from the Standardisation Sub-committee of the International Continence Society. Am J Obstet Gynecol 187, no. 1 (2002): 116-26.

21. Christopher R Chapple et al., The Role of Urinary Urgency and Its Measurement in the Overactive Bladder Symptom Syndrome: Current Concepts and Future Prospects. BJU International 95, no. 3 (February 2005): 335-340.

22. W C de Groat, A Neurologic Basis for the Overactive Bladder. Urology 50, no. 6A Suppl (December 1997): 36-52; discussion 53-56.

23. F Cruz, Desensitization of Bladder Sensory Fibers by Intravesical Capsaicin or Capsaicin Analogs. A New Strategy for Treatment of Urge Incontinence in Patients with Spinal Detrusor Hyperreflexia or Bladder Hypersensitivity Disorders. International Urogynecology Journal and Pelvic Floor Dysfunction 9, no. 4 (1998): 214-220.

24. C J Fowler, M Harper, and C H Fry, Voiding and the Sacral Reflex Arc: Lessons from Capsaicin Instillation. Scandinavian Journal of Urology and Nephrology. Supplementum, no. 210 (2002): 46-50.

25. Katelyne Hubeaux et al., Evidence for Autonomic Nervous System Dysfunction in Females with Idiopathic Overactive Bladder Syndrome. Neurourology and Urodynamics 30, no. 8 (November 2011): 1467-1472.

26. A F Brading, A Myogenic Basis for the Overactive Bladder. Urology 50, no. 6A Suppl (December 1997): 57-67; discussion 68-73.

27. Derek Griffiths and Stasa D Tadic, Bladder Control, Urgency, and Urge Incontinence: Evidence from Functional Brain Imaging. Neurourology and Urodynamics 27, no. 6 (2008): 466-474.

28. Noritoshi Sekido et al., How Many Uncomplicated Male and Female Overactive Bladder Patients Reveal Detrusor Overactivity During Urodynamic Study? International Journal of Urology: Official Journal of the Japanese Urological Association 13, no. 10 (October 2006): 1276-1279.

29. Mohammed A Al-Ghazo et al., Urodynamic Detrusor Overactivity in Patients with Overactive Bladder Symptoms. International Neurourology Journal 15, no. 1 (March 2011): 48-54.

30. Peter Herbison et al., Effectiveness of Anticholinergic Drugs Compared with Placebo in the Treatment of Overactive Bladder: Systematic Review. BMJ (Clinical Research Ed.) 326, no. 7394 (April 19, 2003): 841-844.

31. Manish Gopal et al., Discontinuation Rates of Anticholinergic Medications Used for the Treatment of Lower Urinary Tract Symptoms. Obstetrics and Gynecology 112, no. 6 (December 2008): 1311-1318. 
32. B Schurch, D M Schmid, and M Stöhrer, Treatment of Neurogenic Incontinence with Botulinum Toxin A. The New England Journal of Medicine 342, no. 9 (March 2, 2000): 665.

33. Heinrich Schulte-Baukloh et al., Botulinum-A Toxin Detrusor and Sphincter Injection in Treatment of Overactive Bladder Syndrome: Objective Outcome and Patient Satisfaction. European Urology 48, no. 6 (December 2005): 984-990; discussion 990.

34. Linda Brubaker et al., Refractory Idiopathic Urge Urinary Incontinence and Botulinum A Injection. The Journal of Urology 180, no. 1 (July 2008): 217-222.

35. Gilles Karsenty et al., Botulinum Toxin A (Botox) Intradetrusor Injections in Adults with Neurogenic Detrusor Overactivity/neurogenic Overactive Bladder: a Systematic Literature Review. European Urology 53, no. 2 (February 2008): 275-287.

36. Randall K Leong, Stefan G G De Wachter, and Philip E V van Kerrebroeck, Current Information on Sacral Neuromodulation and Botulinum Toxin Treatment for Refractory Idiopathic Overactive Bladder Syndrome: a Review. Urologia Internationalis 84, no. 3 (2010): 245-253.

37. Vera Vandoninck et al., Percutaneous Tibial Nerve Stimulation in the Treatment of Overactive Bladder: Urodynamic Data. Neurourology and Urodynamics 22, no. 3 (2003): 227-232.

38. Floor van der Pal et al., Percutaneous Tibial Nerve Stimulation in the Treatment of Refractory Overactive Bladder Syndrome: Is Maintenance Treatment Necessary? BJU International 97, no. 3 (March 2006): 547-550.

39. M M Hassouna et al., Sacral Neuromodulation in the Treatment of Urgency-frequency Symptoms: a Multicenter Study on Efficacy and Safety. The Journal of Urology 163, no. 6 (June 2000): 1849-1854.

40. C. J. Fowler et al., Abnormal Electromyographic Activity of the Urethral Sphincter, Voiding Dysfunction, and Polycystic Ovaries: a New Syndrome? BMJ 297, no. 6661 (1988): 1436-1438.

41. M J Swinn et al., Sacral Neuromodulation for Women with Fowler's Syndrome. European Urology 38, no. 4 (October 2000): 439-443.

42. Gabriele Baldini et al., Postoperative Urinary Retention: Anesthetic and Perioperative Considerations. Anesthesiology 110, no. 5 (May 2009): 1139-1157.

43. V. Gruenewald and U. Jonas, Neurostimulation for Lower Urinary Tract Voiding Problems. Curr Urol Rep 1, no. 3 (2000): 199-203.

44. D. B. Vodusek, Interventional Neurophysiology of the Sacral Nervous System. Neurophysiol Clin 31, no. 4 (2001): 239-46.

45. R. A. Schmidt and R. Doggweiler, Neurostimulation and Neuromodulation: a Guide to Selecting the Right Urologic Patient. Eur Urol 34 Suppl 1 (1998): 23-6.

46. E.J. Chartier-Kastler, Long-term Results of Sacral Nerve Stimulation (S3) for the Treatment of Neurogenic Refractory Urge Incontinence Related to Detrusor Hyperreflexia, Journal of Urology 164 (2000): 1476-1480.

47. B Schurch et al., Electrophysiological Recordings During the Peripheral Nerve Evaluation (PNE) Test in Complete Spinal Cord Injury Patients. World Journal of Urology 20, no. 6 (May 2003): 319-322.

48. Wassim Chaabane et al., Sacral Neuromodulation for Treating Neurogenic Bladder Dysfunction: Clinical and Urodynamic Study. Neurourology and Urodynamics 30, no. 4 (April 2011): 547-550.

49. G Lombardi and G Del Popolo, Clinical Outcome of Sacral Neuromodulation in Incomplete Spinal Cord Injured Patients Suffering from Neurogenic Lower Urinary Tract Symptoms, Spinal Cord 47, no. 6 (June 2009): 486-491.

50. K E Matzel et al., Electrical Stimulation of Sacral Spinal Nerves for Treatment of Faecal Incontinence, Lancet 346, no. 8983 (October 28, 1995): 1124-1127.

51. O Uludag et al., Effect of Sacral Neuromodulation on the Rectum. The British Journal of Surgery 92, no. 8 (August 2005): 1017-1023.

52. Klaus E Matzel et al., Sacral Spinal Nerve Stimulation for Faecal Incontinence: Multicentre Study. Lancet 363, no. 9417 (April 17, 2004): 1270-1276.

53. Michael A Kamm et al., Sacral Nerve Stimulation for Intractable Constipation. Gut 59, no. 3 (March 2010): 333-340. 
54. Dimitrios-Anestis Moutzouris and Matthew E Falagas, Interstitial Cystitis: An Unsolved Enigma. Clinical Journal of the American Society of Nephrology: CJASN 4, no. 11 (November 2009): 1844-1857.

55. Elena E Ustinova, Matthew O Fraser, and Michael A Pezzone, Cross-talk and Sensitization of Bladder Afferent Nerves. Neurourology and Urodynamics 29, no. 1 (2010): 77-81.

56. C F Maher et al., Percutaneous Sacral Nerve Root Neuromodulation for Intractable Interstitial Cystitis. The Journal of Urology 165, no. 3 (March 2001): 884-886.

57. Craig V Comiter, Sacral Neuromodulation for the Symptomatic Treatment of Refractory Interstitial Cystitis: a Prospective Study. The Journal of Urology 169, no. 4 (April 2003): 1369-1373. 



\section{Chapter 3}

Five-year results of sacral neuromodulation therapy for urinary voiding dysfunction: Outcomes of a prospective, worldwide clinical study

Philip E.V. van Kerrebroeck, Anco C van Voskuilen, John P.F.A. Heesakkers, A.A.B. Lycklama á Nijholt, Steven Siegel, U. Jonas, Clare J. Fowler, Magnus Fall, Jerzy B. Gajewski, Magdy M Hassouna, Francesco Cappellano, Mostafa M Elhilali, Douglas F. Milam, Anurag K Das, H. Dijkema, Ubi van den Hombergh 


\section{ABSTRACT}

Purpose: This 5-year, prospective, multicenter trial evaluated the long-term safety and efficacy of sacral neuromodulation in patients with refractory urge incontinence (UI), urgency-frequency (UF), and retention.

Material and Methods: A total of 17 centers worldwide enrolled 163 patients (87\% female). Following test stimulation, 11 patients declined implantation, and 152 were implanted using InterStim (Medtronic Inc, Minneapolis). Of those treated with implantation, 96 patients $(63.2 \%)$ had urge incontinence, 25 (16.4\%) had urgency frequency, and 31 (20.4\%) had retention. Voiding diaries were collected annually for 5 years. Clinical success was defined as $50 \%$ or greater improvement from baseline in primary voiding diary variable(s).

Results: Data for all implanted cases were reported.

For patients with urge incontinence, the mean number of leaking episodes per day decreased from $9.6 \pm 6.0$ to $3.9 \pm 4.0$. For patients with urgency-frequency, mean voids per day decreased from $19.3 \pm 7.0$ to $14.8 \pm 7.6$ and mean voided volume per void increased from $92.3 \pm 52.8$ to $165.2 \pm 147.7 \mathrm{ml}$. For patients with retention, the mean volume per catheterization decreased from $379.9 \pm 183.8$ to $109.2 \pm 184.3 \mathrm{ml}$ and the mean number of catheterization decreased from $5.3 \pm 2.8$ to $1.9 \pm 2.8$. All changes were statistically significant $(p<0.0001)$. No life-threatening or irreversible adverse events occurred. In 102 patients 297 device-related or therapy-related adverse events were observed. At 5 years after implantation, $68.0 \%$ of patients with urge incontinence, $56.0 \%$ with urgency frequency and $71.0 \%$ with retention patients had successful outcomes.

Conclusions: This long-term study demonstrates that InterStim therapy is safe and effective for restoring voiding in appropriately selected cases refractory to other forms of treatment. 


\section{INTRODUCTION}

During the last decade, sacral neuromodulation (SNM), often referred to as sacral nerve stimulation (SNM) or InterStim therapy, has proven to be an effective treatment modality for voiding dysfunctions that are refractory to conservative treatment, particularly urge urinary incontinence (UI), urgency-frequency (UF) and idiopathic nonobstructive retention. ${ }^{1-11}$ In Europe, this therapy has also shown excellent clinical outcomes in treating fecal incontinence and idiopathic constipation, but these indications are not approved in the United States. Many patients treated with SNM experience an immediate clinical benefit that is sustained over long-term follow-up. Several authors have published their results with SNM, reporting on various follow-up periods and success rates. As new techniques, devices, and components emerge, analysis of long-term data, particularly those gathered from the original patient groups enrolled in FDA-approved protocol studies, is important to advance our knowledge of SNM.

This study, one of the earliest and largest prospective, multicenter clinical trials of SNM therapy, has concluded after 5 years of follow-up. We report here the longterm results.

\section{MATERIAL AND METHODS}

This Post-Approval Study (PAS) was a prospective, nonrandomized, multicenter, follow-up study initiated after U.S. Food and Drug Administration (FDA) approval of InterStim Therapy. Most of the enrolled patients (129) were already implanted with the InterStim system for the treatment of UI, UF, and nonobstructive urinary retention as part of the multicenter, randomized controlled study (MDT-103) and were consecutively crossed-over to the long-term follow-up study. All 17 Ethical Committees approved the long-term PAS protocol and each patient signed an informed consent form before study participation. Six of the 23 centers participating in the original randomized study ${ }^{1,2}$ (MDT-103) declined to participate in the PAS and those patients were excluded from further follow-up. No significant differences between patients from the declined and the participating centers could be identified.

\section{Patient selection and evaluation}

The PAS study enrolled a total of 163 patients. Demographic data of 1 patient was not available. The mean age was 44.7 years \pm 11.2 years $(17.5-78.9)$ with 21 males (13\%) and 141 females (87\%). By indication 103 patients (63.6\%) had UI, 28 (17.3\%) 
had UF, and 31 (19.1\%) suffered from complete or incomplete urinary retention. A total of 129 patients crossed-over from the MDT-103 study and 34 were enrolled under the new PAS protocol. Of these 34 patients, 23 were implanted with the InterStim system and 11 declined a permanent implant despite a positive response to trial stimulation. The five-year results for all 152 patients are reported.

The PAS study used a patient follow-up protocol similar to the original MDT-103 study. ${ }^{12,13}$ Compared to the MDT-103 study, the PAS required annual follow-up visits, and used more advanced devices (test stimulation leads, implantable neurostimulators) and new techniques (neurostimulator implant in the buttock). The majority of patients had the INS implanted in the abdomen (121) and only 31 patients who were enrolled later on, had the INS implanted in the buttock. The criteria for rating clinical success based on patients' voiding diaries and the methods for reporting and analyzing $A E^{\prime}$ 's remained unchanged. The required data at the annual follow-up visits for all implanted patients who underwent implantation included a voiding diary completed for a minimum of 3 days, and simple Uroflow and quality-of-life questionnaires. The questionnaires used were the Short Form-36 (SF-36) and the Beck Depression Index (BDI). Detailed data were also collected on any concomitant treatment for the urological condition and on any therapy or patient related complication.

The voiding dysfunction indications (UI, UF, and retention) were represented by unique primary voiding diary variables on which therapy outcome was measured. For UI patients, these voiding diary variables included: number of leaking episodes daily, number of heavy leaking episodes daily, and number of pads used daily.

For patients with UF, primary voiding diary variables included: number of voids daily, volume voided per void and degree of urgency experienced before void.

For patients with retention, primary voiding diary variables included: catheterized volume per catheterization and number of catheterizations daily. InterStim Therapy was considered successful if a minimum of $50 \%$ improvement occurred in any of primary voiding diary variables compared with the baseline. Safety data were reported for all cases. Descriptive summaries were provided for device or therapy related $A E^{\prime}$ s and also those requiring surgical intervention.

\section{Patient Disposition}

In this long term follow-up study patients were followed up for 5 years. Due to the long follow-up period, some scheduled visits were missed and not all patients provided a voiding diary at each follow-up visit. Table 1 provides a breakdown of the follow-up elements by patient group (numbers of visits, diaries and explants) at each follow-up period. A total of 138 patients completed 1-year follow-up and 121 patients completed the 1-year voiding diary. At 5 years a total of 105 patients com- 
pleted follow-up and 87 completed the voiding diary. There were 47 patients who did not have 5-year data, and 16 of them had the system explanted and withdrew due to lack of efficacy or complications. The last follow-up visit with diary data occurred on average at 49.3 months 15.9 (range 11 to 60) following implantation for the 145 patients with at least 1 follow-up diary past 12 months.

\section{Statistical Analysis}

For each of the primary voiding diary parameters relevant for measurement of UI, UF, and retention, statistical comparisons were made between baseline and post implant using a repeated measure analysis. A clinical success rate (percentage of patients with $50 \%$ or more improvement in the voiding diary variable) at each visit was calculated for each primary voiding diary variable.

Patients who exited the study due to an adverse event or lack of efficacy were considered unsuccessful and were assumed to return to their baseline.

For patients who missed a study visit or failed to provide a voiding diary at a followup visit for other reasons, the last observation carried forward was used to impute missing data. A sensitivity analysis based on all implanted cases with valid follow-up and diary data were also performed to assess the robustness of the results.

Table 1. Breakdown of follow-up elements by post implant visit.

\begin{tabular}{|c|c|c|c|c|c|c|}
\hline Indication & $\begin{array}{l}\text { Follow-up } \\
\text { (Number) }\end{array}$ & $\begin{array}{l}\text { Month } \\
12\end{array}$ & $\begin{array}{c}\text { Month } \\
24\end{array}$ & $\begin{array}{l}\text { Month } \\
36\end{array}$ & $\begin{array}{l}\text { Month } \\
48\end{array}$ & $\begin{array}{c}\text { Month } \\
60\end{array}$ \\
\hline Urge & Visits & 87 & 79 & 72 & 69 & 65 \\
\hline Incontinence & Diaries & 71 & 65 & 61 & 57 & 54 \\
\hline $\begin{array}{l}\text { (Baseline, } \\
\mathrm{N}=96)\end{array}$ & $\begin{array}{l}\text { Explants due to complication or } \\
\text { lack of efficacy (cumulative) }\end{array}$ & 1 & 2 & 3 & 4 & 8 \\
\hline Retention & Visits & 27 & 26 & 28 & 28 & 27 \\
\hline (Baseline, & Diaries & 27 & 24 & 26 & 19 & 22 \\
\hline$N=31)$ & $\begin{array}{l}\text { Explants due to complication or } \\
\text { lack of efficacy (cumulative) }\end{array}$ & 0 & 0 & 0 & 1 & 1 \\
\hline Urgency / & Visits & 24 & 19 & 16 & 16 & 13 \\
\hline Frequency (Base- & - Diaries & 23 & 19 & 14 & 16 & 11 \\
\hline $\begin{array}{l}\text { line, } \\
\mathrm{N}=25 \text { ) }\end{array}$ & $\begin{array}{l}\text { Explants due to complication or } \\
\text { lack of efficacy (cumulative) }\end{array}$ & 0 & 2 & 5 & 6 & 7 \\
\hline
\end{tabular}




\section{RESULTS}

\section{Efficacy for Urge incontinence}

The average number of leaking episodes per day at baseline for the patients with (UI) was $9.6 \pm 6.0$. This decreased to $4.7 \pm 4.9$ at 1-year follow-up and the reduction was maintained throughout follow-up with an average of $3.9 \pm 4.0$ at 5 years after implantation. (Part A of figure 1)

The number of heavy leaks per day decreased from $2.6 \pm 3.3$ at baseline to $1.2 \pm 2.7$ leaks at 1-year follow-up, and to $0.8 \pm 1.7$ at 5 years after implantation.

Patients with urge incontinence also showed a decrease in the number of pads used per day, from more than 5 at baseline to 1.8 at at 5 years after implantation. $A$ clinical success rate of $58 \%$ was observed in number of leaks per day, $68 \%$ in number of heavy leaks per day and $61 \%$ in number of pads used per day at 5 -year follow-up (Table 2).

Table 2. Clinical success rates for 5 years

\begin{tabular}{|c|c|c|c|c|c|}
\hline \multicolumn{6}{|c|}{ Clinical success rates for 5 years after implantation by indication and visit } \\
\hline & 1-Year & 2-Year & 3-Year & 4-Year & 5-Year \\
\hline \multicolumn{6}{|l|}{$\%$ UI (No/Total No): } \\
\hline Leaking episodes per day & $51.0 \%(49 / 96)$ & $60.4 \%(58 / 96)$ & $59.4 \%(57 / 96)$ & $58.3 \%(56 / 96)$ & $58.3 \%(56 / 96)$ \\
\hline Heavy leaking episodes per day & $50.0 \%(40 / 80)$ & $60.8 \%(48 / 79)$ & $66.7 \%(52 / 78)$ & $68.0 \%(53 / 78)$ & $68.0 \%(53 / 78)$ \\
\hline Pads per day & $51.1 \%(45 / 88)$ & $59.6 \%(53 / 89)$ & $53.9 \%(48 / 89)$ & $57.5 \%(50 / 87)$ & $60.9 \%(53 / 87)$ \\
\hline \multicolumn{6}{|l|}{$\%$ UF (No/Total No): } \\
\hline Voids/day & $40.0 \%(10 / 25)$ & $48.0 \%(12 / 25)$ & $52.0 \%(13 / 25)$ & $48.0 \%(12 / 25)$ & $40.0 \%(10 / 25)$ \\
\hline Vol/void & $60.0 \%(15 / 25)$ & $68.0 \%(17 / 25)$ & $64.0 \%(16 / 25)$ & $56.0 \%(14 / 25)$ & $56.0 \%(14 / 25)$ \\
\hline Degree of urgency & $68.0 \%(17 / 25)$ & $72.0 \%(18 / 25)$ & $68.0 \%(17 / 25)$ & $60.0 \%(15 / 25)$ & $56.0 \%(14 / 25)$ \\
\hline \multicolumn{6}{|l|}{ \% Retention (No/Total No): } \\
\hline Catheters/day & $67.7 \%(21 / 31)$ & $64.5 \%(20 / 31)$ & $67.7 \%(21 / 31)$ & $67.7 \%(21 / 31)$ & $58.1 \%(18 / 31)$ \\
\hline Vol/Catheter & $67.7 \%(21 / 31)$ & $77.4 \%(24 / 31)$ & $77.4 \%(24 / 31)$ & $71.0 \%(22 / 31)$ & $71.0 \%(21 / 31)$ \\
\hline
\end{tabular}




\section{UI Patients}

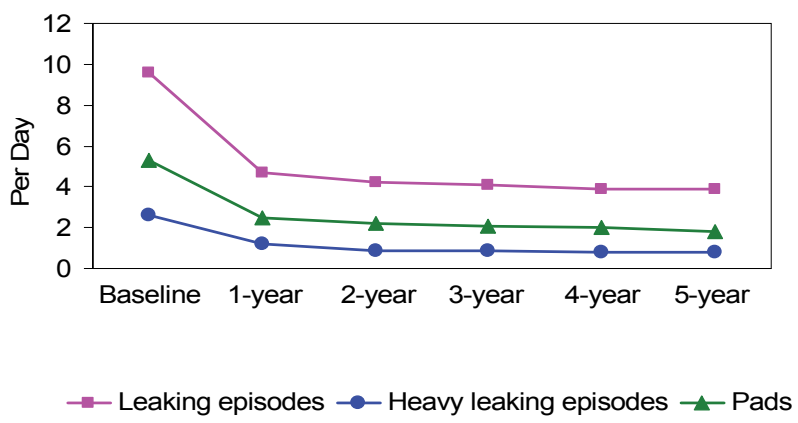

UF Patients

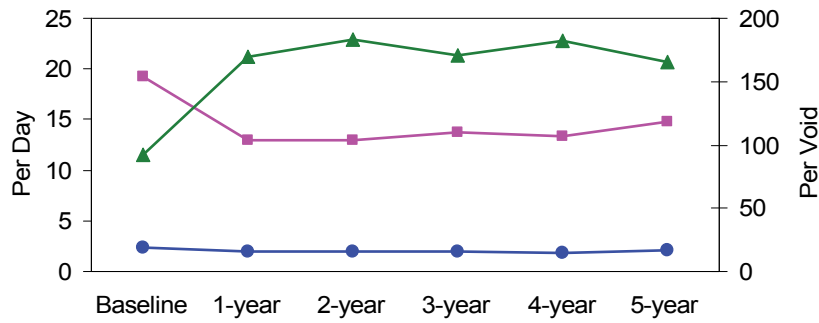

$\rightarrow$ Voids $\rightarrow$ - Degree of urgency $\rightarrow$ Vol voids

\section{Retention Patients}

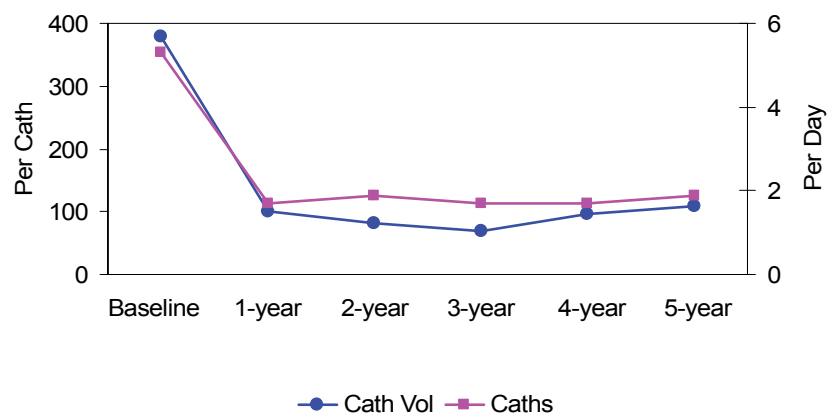

Figure 1. Average voiding diary parameters during 5 years after implant. A, patients with UI. B, patients with UF. C, patients with retention. Missing values were imputed using last observation carried forward. Except for degree of urgency, voiding parameter changes were statistically significant $(p<0.0001)$ from repeated measure analysis. 


\section{Efficacy for Urgency-Frequency}

The average number of voids per day decreased from $19.3 \pm 7.0$ at baseline to 13.0 \pm 7.9 at 1 -year follow-up. This reduction was maintained throughout the long-term follow-up period with a slight increase to $14.8 \pm 7.6$ at 5 years after implantation (Part B of Figure 1). The average voided volume increased from $92.3 \pm 52.8 \mathrm{ml}$ at baseline to $169.9 \pm 118.2 \mathrm{ml} 1$ year after implantation and effectiveness was maintained throughout the long-term follow-up period with average of $165.2 \pm 147.7$ at 5 years after implantation. Patient with urgency/frequency patients reported a decrease in degree of urgency per day from $2.3 \pm 0.6$ at baseline to $1.9 \pm 0.8$ at oneyear follow-up and $2.1 \pm 0.7$ at 5 -year follow-up. A clinical success rate of $40 \%$ was observed in number of voids per day and $56 \%$ in degree of urgency per day at 5 years after implantation for patients with UF, and $56 \%$ experienced a $50 \%$ or more increase in average volume voided per void.

Of the patients with a successful clinical outcome after 1 year implant $71 \%$ remained successful at 5 year follow-up.

\section{Efficacy for Urinary Retention}

The average number of catheterizations per day decreased from $5.3 \pm 2.8$ at baseline to $1.9 \pm 2.8$, and $58 \%$ clinical efficacy was observed 5 after implantation. The average catheterized volume per catheterization decreased from $379.9 \pm 183.8 \mathrm{ml}$ at baseline to $109.2 \pm 184.3 \mathrm{ml}$ and clinical success rate of $71 \%$ was observed 5 years after implantation (Part $\mathrm{C}$ of Figure 1 ).

Of those patients with an unsuccessful therapy outcome the therapy failure period ranged over 6-60 months post-implantation, with a mean failure period of 24 months for all indications. To assess the robustness of reported results, sensitivity analysis was performed based on all evaluable patients with valid visits and diary data. Consistent results were obtained and, thus, not presented.

The quality of life scores (Short Form-36 and Beck Depression Inventory) showed some improvement, but this was not statistically significant.

Interpretation of these data is limited because of the generic nature of the selected questionnaires which were the only methods available at the time the study was designed.

\section{Safety}

The safety data were reported based on all implanted cases. There were 102 (67\%) patients who had at least 1 device or therapy related adverse event. A total of 221 AEs were recorded and most (96\%) were resolved by the time the data was analyzed. A total of 110 adverse events required surgery in 60 patients. There were 92 
patients analyzed who underwent implantation and did not have adverse events requiring surgical intervention. Overall, the system was explanted from 16 patients due to adverse event or lack of efficacy.

Of the adverse events in 102 patients, 31 were device-related (24 patients, 15.8\%), and 240 were therapy-related (97 patients, 63.8\%) (Table 3).

Among the 240 therapy-related events, the most frequently reported event was new pain or undesirable change in stimulation, which occurred 60 times in 41 patients $(27.0 \%$ of all patients). Pain at the percutaneous nerve evaluation (PNE) or the implant site related to the implantable neurostimulator (INS) was the second most commonly reported event, occurring 40 times in 30 patients ( $19.7 \%$ of all patients).

Of the device-related adverse events, the most frequently reported were suspected device problems (5.3\%) and suspected lead migration (3.3\%) (Table 3).

Throughout the 5-year follow-up period, surgical intervention was required to resolve adverse events in 60 patients ( 110 events).

Device exchange, which included replacement of the lead, the extension, or the INS, was the most common intervention, resulting from 52 adverse events in 36 patients (23.7\%). The types of surgical interventions performed are shown in Table 4, including the percentage of patients who experienced an adverse event requiring those interventions. These figures include a group of patients who had an INS exchange because of battery depletion. A total of 60 (39.5\%) patients experienced adverse event requiring device exchange. Of the 152 patients who were implanted and analyzed, 92 (60.5 \%) did not have an adverse event requiring surgical intervention. 
Table 3. Device or therapy related adverse events

\begin{tabular}{lcccc}
\hline Adverse Event & $\begin{array}{c}\text { Device Related } \\
\text { No. of Pt. With } \\
\text { AEs (\%) }\end{array}$ & $\begin{array}{c}\text { No. Cumula- } \\
\text { tive AEs }\end{array}$ & $\begin{array}{c}\text { No. of Pt. With } \\
\text { AEs (\%) }\end{array}$ & $\begin{array}{c}\text { No. Cumula- } \\
\text { tive AEs }\end{array}$ \\
\hline New pain/undesirable change in & $2(1.3)$ & 2 & $41(27.0)$ & 58 \\
stimulation & & & & \\
Pain at PNE or implant site - INS & $1(0.7)$ & 1 & $29(19.1)$ & 39 \\
Suspected device problem & $8(5.3)$ & 5 & $8(5.3)$ & 8 \\
Suspected lead migration & $5(3.3)$ & 0 & $12(7.9)$ & 9 \\
Infection-PNE or implant site & 0 & 2 & $10(6.6)$ & 14 \\
Sensation of electric shock & $2(1.3)$ & 0 & $12(7.9)$ & 13 \\
Pain at PNE or implant site - Lead & 0 & 0 & $11(7.2)$ & 12 \\
Undesirable change in voiding function & 0 & 0 & $8(5.3)$ & 8 \\
Technical problems during PNE/implant & 0 & 10 & $44(29.0)$ & 67 \\
Other & $7(4.6)$ & 31 & $97(63.8)$ & 240 \\
Total & $24(15.8)$ & $5.3)$ & 12 \\
\hline
\end{tabular}

Table 4. Device or therapy related adverse events requiring surgical intervention

\begin{tabular}{lcc}
\hline $\begin{array}{l}\text { Device or therapy related adverse events requiring surgical intervention } \\
\text { Surgical Intervention }\end{array}$ & $\begin{array}{c}\text { No patients with AEs (\%) } \\
\text { No cumulative AEs }\end{array}$ \\
\hline Device exchange & $36(23.7)$ & 52 \\
Reposition of the IPG & $12(7.9)$ & 16 \\
Reposition Lead and IPG & $10(6.6)$ & 14 \\
Reposition Lead & $10(6.6)$ & 12 \\
Permanent Explant & $9(5.9)$ & 10 \\
Temporary Explant & $2(1.3)$ & 2 \\
Other Surgical Intervention & $2(1.3)$ & 1 \\
Bilateral Implant & $1(0.7)$ & 1 \\
Surgical Wound Care & $1(0.7)$ & 110 \\
Any Adverse Event & $60(39.5)$ & 2 \\
\hline
\end{tabular}

\section{DISCUSSION}

This long-term, prospective, multicenter, FDA-regulated study represents the most comprehensive follow-up study of sacral neuromodulation therapy with the largest patient population to be analyzed or published to date.

The efficacy data confirm the sustained clinical benefit of sacral neuromodulation that has been shown in various patient populations reported in recent years. ${ }^{2,9,10,14}$ A drawback of such a long-term trial is that they cannot assess new therapeutic advancements that occur after the study begins, which may potentially affect patient selection, procedure techniques, devices used, and overall patient outcomes. 
Another disadvantage of the study is the missing data due to patients not attending at scheduled visits or becoming lost to follow up.

Therefore, clinical implication from this study should be interpreted with caution although a sensitivity analysis was performed based on all evaluable data. Nonetheless, this study demonstrates that approximately $70 \%$ of the treated patients continued to experience therapeutic benefit after 5 years of the treatment. The approximately $30 \%$ of the patients who did not benefit from the treatment between 6 and 24 months follow-up is consistent with previously published literature. $^{10}$

Many possible explanations exist for treatment failure, including potential placebo effect of trial stimulation, insufficient test stimulation sensitivity before implantation and inadequate patient selection (e.g., severe disease state at the time of the study enrollment, presence of specific mental conditions, and previous medicosocial history). For instance, Weil et al, reporting on their long-term results in 36 patients, found a striking correlation between secondary loss of therapeutic effect and the presence of a pre-existing psychiatric disorder in the medical history. ${ }^{9}$ However, the effect of psychiatric disorders on the efficacy of neuromodulation has not been established.

An important finding in this study is the high correlation between the 1- and 5-year success rates for treated patients. There were $84 \%$ patients with UI, $71 \%$ with UF, and $78 \%$ with urinary retention who were successfully treated at 1-year follow-up continued to have a successful outcome at 5 years after implantation.

SNM has proved to be a safe therapy that does not preclude any other treatment options. The mid-term safety profile of SNM was established in the original MDT103 study with 219 implanted cases. ${ }^{12}$ No reports of any life-threatening or irreversible adverse events have been made in this or any previous studies of neuromodulation for urinary conditions. Of the 271 reported adverse events in this study, 110 required surgery in 60 patients. The most common surgical interventions were device exchange or device repositioning, which are generally considered minor surgical procedures. Pain at the PNE or implant site necessitating surgical intervention was the most common indication occurring in $11.8 \%$ of implanted patients throughout the course of the study. The second and third most common indications requiring surgical intervention were suspected lead migration and new pain or undesirable change in stimulation. As 12 of the 14 patients experiencing these adverse events had undergone implantation with an older Itrel-II INS, which has been superseded by the new InterStim INS, the mid-term and long-term number of devicerelated surgical interventions is expected to decrease. INS exchange due to battery 
depletion will continue to occur, on average, every 8 years. However, this is a minor intervention performed on an outpatient basis under local anesthesia.

In 1999, the placement of the INS was changed from the abdomen to the buttock because buttock placement resulted in less pain and fewer infections postoperatively, which minimized the need for INS repositioning, and reduced operating time. ${ }^{15}$ Despite the lack of randomized controlled trials on the effect of buttock versus abdominal placement and its impact on the rate of surgical re-operation, clinical data seems to confirm convincingly a reduced rate of INS-related complaints. Analysis of the occurrence of surgically corrected adverse events in both treatment groups in our study revealed that the re-operation rate due to pain at the INS site decreased in cases of buttock placement. The difference was not statistically significant due to the relatively small number of patients who were enrolled in the study with the buttock neurostimulator implant.

At 1-year follow-up post implant, $19.9 \%$ of the patients had adverse event resulting in surgical intervention, which increased to $42.1 \%$ by 5-years of follow-up. Of 152 implanted cases, 92 did not have an adverse event requiring surgical intervention. Although this is a relatively high number, we emphasize that this was the first study in which SNM therapy was offered to quite severely affected patients and with steep learning curve.

It should also be noted that patients, who experienced the loss of therapeutic effect, often underwent revision surgery because it was believed at the time that new lead placement or switching to the contralateral side would restore benefit. Revision surgeries were performed and repeated on each patient when a technical problem occurred, the device had to be replaced, or when pain complaints indicated a problem. A later study demonstrated that loss of therapeutic effect often cannot be resolved with revision surgery. ${ }^{14}$

Patient selection and the SNM operating technique involved a steep learning curve. Some of the devices and surgical techniques used early in the study have been replaced by major advancements within the last 3 years. For example, the technique has become minimally invasive with the use of percutaneous lead placement under local anesthesia that allows patient participation to help determine sensory responses. Reported adverse event rates from recent literature are between 11 and $22 \%$ Specifically for surgical revision, the rates change from $7 \%$ to $18 \%$ with followup from 7 to 24 months. ${ }^{16-18}$ This clearly demonstrates a reduction in the adverse event rates with the new technology (minimally invasive technique and the use of the tined lead and upper buttock INS placement) Much has been learned at each centre regarding the requirements for success, including the need for experienced personnel and appropriate hospital set-up for optimal therapy delivery. These improvements are now being practiced in all centres providing SNM therapy. 


\section{CONCLUSION}

This long-term study dramatically advanced overall knowledge of sacral neuromodulation therapy for urinary dysfunction. The results show that sacral neuromodulation safely and effectively provides long-term relief for appropriately selected patients with refractory UI, UF, and urinary retention. SNM is a safe, reversible, minimally invasive method that may be an appropriate alternative to more invasive techniques for well selected patients. Although much has been learned, methods can still be optimized. Further research is warranted to elicit the mechanism of action, to improve patient selection and the sensitivity of percutaneous trial stimulation, and to avoid adverse events after implantation.

\section{REFERENCES}

1. Siegel, S. W.: Management of voiding dysfunction with an implantable neuroprosthesis. Urologic Clinics of North America, 19: 163, 1992

2. Hassouna, M. M., Siegel, S. W., Nyeholt, A. A. et al.: Sacral neuromodulation in the treatment of urgency-frequency symptoms: a multicenter study on efficacy and safety. Journal of Urology, 163: 1849, 2000

3. Dijkema, H. E., Weil, E. H., Mijs, P. T. et al.: Neuromodulation of sacral nerves for incontinence and voiding dysfunctions. Clinical results and complications. European Urology, 24: 72, 1993

4. Bosch, J. L., Groen, J.: Sacral (S3) segmental nerve stimulation as a treatment for urge incontinence in patients with detrusor instability: results of chronic electrical stimulation using an implantable neural prosthesis. Journal of Urology, 154: 504, 1995

5. Hasan, S. T., Shanahan, D. A., Pridie, A. K. et al.: Surface localization of sacral foramina for neuromodulation of bladder function. An anatomical study. European Urology, 29: 90, 1996

6. Hasan, S. T., Robson, W. A., Pridie, A. K. et al.: Transcutaneous electrical nerve stimulation and temporary S3 neuromodulation in idiopathic detrusor instability. Journal of Urology, 155: 2005, 1996

7. Shaker, H. S., Hassouna, M.: Sacral root neuromodulation in idiopathic nonobstructive chronic urinary retention. Journal of Urology, 159: 1476, 1998

8. Shaker, H. S., Hassouna, M.: Sacral nerve root neuromodulation: an effective treatment for refractory urge incontinence. Journal of Urology, 159: 1516, 1998

9. Weil, E. H., Ruiz Cerda, J. L., Eerdmans, P. H. et al.: Clinical results of sacral neuromodulation for chronic voiding dysfunction using unilateral sacral foramen electrodes. World Journal of Urology, 16: 313,1998

10. Bosch, J. L., Groen, J.: Sacral nerve neuromodulation in the treatment of patients with refractory motor urge incontinence: long-term results of a prospective longitudinal study. Journal of Urology, 163: 1219, 2000

11. Koldewijn, E. L., Rosier, P. F., Meuleman, E. J. et al.: Predictors of success with neuromodulation in lower urinary tract dysfunction: results of trial stimulation in 100 patients. Journal of Urology, 152: 2071, 1994

12. Siegel, S. W., Catanzaro, F., Dijkema, H. E. et al.: Long-term results of a multicenter study on sacral nerve stimulation for treatment of urinary urge incontinence, urgency-frequency, and retention. Urology, 56: 87, 2000

13. Janknegt, R. A., Hassouna, M. M., Siegel, S. W. et al.: Long-term effectiveness of sacral nerve stimulation for refractory urge incontinence. European Urology, 39: 101, 2001 
14. Everaerdt, K., De Ridder, D., Baert, L., Oosterlinck, W., Wijndaele, J. J.: Patient satisfaction and complications following sacral nerve stimulation for urinary retention, urge incontinence and perineal pain: A multicenter evaluation. Int Urogynaecol J, 11: 231, 2000

15. Scheepens, W. A., Weil, E. H., van Koeveringe, G. A. et al.: Buttock placement of the implantable pulse generator: A new implantation technique for sacral neuromodulation--a multicenter study. European Urology, 40: 434, 2001

16. Starkman JS, Wolter CE, Scarpero HM, Milam DF, Dmochowski RR. Management of refractory urinary urge incontinence following urogynecological surgery with sacral neuromodulation. Neurourol Urodyn. 2007;26:29-35;

17. Van Voskuilen AC, Oerlemans DJ, Weil EH, van den Hombergh $U$, van Kerrebroeck PE. Medium-term experience of sacral neuromodulation by tined lead implantation. BJU Int. 2007 Jan;99:107-10.

18. Kessler TM, Buchser E, Meyer S, Engeler DS, Al-Khodairy AW, Bersch U, Iselin CE, Roche B, Schmid DM, Schurch B, Zrehen S, Burkhard FC. Sacral neuromodulation for refractory lower urinary tract dysfunction: results of a nationwide registry in Switzerland. Eur Urol. 2007 May;51:1357-63. 


\section{Chapter 4}

Long Term Results of Neuromodulation by Sacral Nerve Stimulation for Lower Urinary

Tract Symptoms:

a Retrospective Single Center Study

A.C. van Voskuilen, D.J.A.J Oerlemans, E.H.J. Weil, R. A. de Bie and Ph.E.V.A. Van Kerrebroeck

Eur Urol. 2006 Feb;49(2):366-72 


\section{ABSTRACT}

Objective: To analyze the influence of technical improvements of Sacral Nerve Stimulation (SNS) on the incidence of surgical interventions and subjective longterm results of SNS.

Methods: Retrospective, transversal study analyzing records of implanted patients at our department of Urology.

Results: Of 149 patients analyzed, 107 had overactive bladder symptoms and 42 had urinary retention. Mean follow-up was 64.2 ( $\mathrm{sd}=38.5$ ) months. In the whole group 194 adverse events occurred. 6 Patients had infection in their implanted system, but none was explanted for infection. Most events could be solved by giving advice or by reprogramming the stimulator. 129 reoperations have been performed and 21 patients had their system explanted. Analysis of the data shows a striking difference in the incidence of reoperations, but small differences in subjective results in the groups of patients implanted before or after 1996, suggesting that a proactive approach towards adverse events is worthwhile.

Conclusions: SNS gives lasting benefit in patients with refractory symptoms of overactive bladder and non-obstructive urinary retention. The differences in outcomes and incidence of re-operation can be attributed to the learning curve and technical and surgical improvements in the application of SNS. 


\section{INTRODUCTION}

In 1994 neuromodulation by sacral nerve stimulation (SNS) became available as a new treatment option for patients with refractory urge incontinence, urgencyfrequency and nonobstructive urinary retention. ${ }^{1,2,3,4}$ The SNS procedure is relatively non-mutilating compared to other surgical procedures. Therefore, it gained a place in the list of therapeutic options a physician can offer to patients with the above-mentioned symptoms. Sacral neuromodulation is placed between noninvasive therapies such as anticholinergics or pelvic floor training and surgical procedures, such as bladder augmentation and urinary deviation. Initially patients were implanted according to the protocol of a multicenter study initiated by the manufacturer of the implanted equipment in order to achieve FDA approval (MDT103). ${ }^{5,6,7,8}$ From the early application of SNS until now continuous research is carried out to improve this therapy and to determine the exact mechanism of action. This research has yielded some technical improvements in SNS. In the period covering our study the design of the Implantable Pulse Generator (IPG) has been changed two times, lead design has changed and there have been advancements in surgical techniques. In our experience these advancements have contributed to decreased complications rates as well as increased success rates in our patients receiving SNS therapy. ${ }^{9,10,11}$ This study is set up to confirm this hypothesis.

\section{METHODS}

This is a transversal cohort study. The surgery logs of the department of Urology of the University Hospital Maastricht covering the period between 1990 and December 2003 have been scrutinized for patients who either received a new definitive neuromodulation system or had revision surgery. Patients were included if they had follow-up of longer than one year and if complete data were available. All patients were implanted according to the open procedure as proposed by Tanagho and Schmidt. ${ }^{12}$ Patients who were implanted through the percutaneous tined lead procedure were excluded, ${ }^{9}$ as well as patients receiving bilateral stimulation. All patients had shown successful on screening using the Percutaneous Nerve Evaluation (PNE) with a temporary lead or after a two-stage procedure. ${ }^{13} 12$ patients had their definitive implant through a 2-stage procedure.

The first series of patients were implanted with the Itrell-I IPG, which was the first model used for SNS. Parameter change in this model was done in an open surgical 
procedure. The Itrell-II was introduced in 1994 and allowed parameter modification by the physician using an external programmer.

Patients, implanted with the Itrell-II could switch the IPG on and off and choose between two preprogrammed settings by using a magnet.

Since 1999 the Interstim IPG is used. This latest model is supplied with a patient remote control for switching the device on and off and for changing amplitude within a preprogrammed range. In our opinion this decreases the outpatient visits as patients can handle amplitude related events by themselves.

The first model of the implanted lead had separate fixation connectors, which had to be sutured onto the lead. In 1994 a new lead model was introduced which had fixation connectors integrated into the lead.

From 2000 the IPG was implanted gluteally instead of abdominally, as this placement gave less complications. ${ }^{10}$

From the files of the implanted patients the following data were collected:

- type of complaints;

- date of implantation;

- date and result of neuromodulation of last check-up;

- number of adverse events and if adverse events had successfully been addressed;

- date of reoperations for adverse events.

Patient complaints were stratified on an 'urge' category and a 'retention' category. The urge category comprises patients suffering from urge incontinence or urgencyfrequency with or without pelvic pain complaints. The retention category consists of patients with non-obstructive retention, regardless of the cause of the complaints. The subjective results of the patients at their last visit were categorized as 'good' or as 'insufficient'. The criteria for success were complete and lasting disappearance of symptoms or satisfactory symptoms for the patient.

An insufficient result was noted when the patient was not satisfied, when symptoms recurred, in the case of an uncorrected adverse event or when the system was explanted.

If at the last follow-up visit the stimulation parameters of the IPG were changed, the chart of the patient was analyzed a second time during the revision period of this manuscript and the result was noted. This is based on the fact that patients are instructed to report back if the symptoms do not improve. The patients are encouraged to contact the clinic when they suspect neuromodulation-related complaints.

The number of adverse events the patient had in the period from implant until last follow-up was counted as well. An adverse event was noted when the patient had neuromodulation-related complaints, not solved by changing the stimulation 
amplitude. Necessary outpatient reprogramming sessions of the stimulator for loss of sensation were only recorded as an adverse event if the loss of sensation was accompanied by loss of efficacy. Battery depletion of the IPG was recorded separately if the procedure was not combined with other neuromodulation related surgery.

The data of the patients were analyzed using SPSS 11.5 for Windows (SPSS inc., USA).

Analysis of contributing factors to the results was done using bivariate correlation analysis by Pearson's Chi-square test. Further statistical analysis was performed using the Mann-Whitney test.

\section{RESULTS}

Between October 1990 and December 2002190 patients were implanted. 15 patients were implanted less than a year ago, and therefore not analyzed. The data in the files of 26 patients was incomplete. In 17 of these files data on the implant was missing as these patients were implanted in another hospital and had reoperations in our department. In the remaining 9 files other data on adverse events or reoperations were missing. A total of 149 patients met the inclusion criteria and were analyzed. The group consisted of 27 male (18.1\%) and 122 female patients (81.9\%). Among these patients 129 (86.6\%) had an idiopathic cause of their symptoms and $20(13.4 \%)$ patients had a neurologic cause of the urinary complaints.

Table 1 shows the diagnosis and the results of these 20 patients. Indications for implant were overactive bladder symptoms in 107 (71.8\%) and retention in 42 (28.2\%) patients.

Mean age at implantation was $46.7(s d=10,0)$ years, range between 21 and 72 years.

Follow-up ranged between 12 and 154 months after implant, the mean follow-up period was $64.2(\mathrm{sd}=38.5)$ months. 
Table 1. The diagnosis of the 20 patients who have a neurologic cause of their urinary complaints and theirs results. ALS: Amyotrophic Lateral Sclerosis, IPD: Intevertebral Disk Prolaps, SCI: Spinal Cord Injury

\begin{tabular}{lccc}
\hline Diagnosis & N of patients & \multicolumn{2}{c}{ Result } \\
& & Good & Insufficient \\
\hline ALS & 1 & 1 & 0 \\
Cauda Equina Syndrome & 3 & 1 & 2 \\
Peripheral polyneuropathy & 2 & 2 & 0 \\
Guillain Barre & 1 & 1 & 0 \\
IDP & 5 & 3 & 2 \\
SCl & 1 & 1 & 0 \\
Multiple Sclerosis & 1 & 1 & 0 \\
Paraplegia & 1 & 0 & 1 \\
Spina Bifida & 1 & 1 & 0 \\
Spondyolysis & 1 & 1 & 0 \\
Whiplash & 2 & 1 & 1 \\
Unknown neurologic & 1 & 0 & 1 \\
Total & $20(100 \%)$ & $13(65 \%)$ & $7(35 \%)$ \\
\hline
\end{tabular}

The mean follow-up for patients who were implanted for urgency symptoms was 69.8 months $(s d=41.6)$ and for patients with urinary retention 70.5 months. $(s d=38.0)$. In the whole group $89(59.7 \%)$ patients were scored as having a good result, $44(29.5 \%)$ patients had insufficient results and 16 patients (10.7\%) had a reprogramming session at their last visit.

During the revision process of this manuscript the follow-up of these 16 patients after database closure showed that $11(68.8 \%)$ had good results after a period of three to 6 months after their visit and $5(31.3 \%)$ patients kept insufficient results after reprogramming.

With these 16 patients included a total of 100 patients (67.1\%) had a good result and $49(32.9 \%)$ had insufficient results. 68 (63.6\%) of the 107 patients with urgency had good results at the time of their last follow-up and 39 (36.4\%) patients with urgency had insufficient results.

In the retention group of 42 patients 32 (76.2\%) patients had good results and 10 patients (23.8\%) had insufficient results.

In the group of 149 patients with a total of 9,562 months of SNS experience 194 adverse events occurred in 106 patients.

There were no irreversible or severe adverse events. The most frequently occurring adverse events were changes in stimulation sensation, loss of efficacy and pain at the Implantable Pulse Generator (IPG) site. 
Table 2. Description of adverse events in our follow-up.

\begin{tabular}{lc}
\hline Adverse event & $\#$ \\
\hline Pain / undesirable change in stimulation & 64 \\
Undesirable change in voiding function/ Loss of efficacy & 42 \\
Pain at IPG implant site & 41 \\
Adverse change in bowel function & 15 \\
Suspected lead migration & 10 \\
Suspected device problem (including lead breakage) & 6 \\
Infection & 6 \\
Technical problem & 5 \\
Suspected neuropraxia & 2 \\
Other & 3 \\
Total & 194 \\
\hline
\end{tabular}

The figures described in this table are numbers of adverse events. Some patients had more than one adverse event. Pain or undesirable stimulation was only recorded as an adverse event when changing the stimulator amplitude could not solve the problem. Suspected neuropraxia was seen in 2 patients who set their amplitudes very high and was in both patients successfully solved by switching the IPG off for 6 months. The events in the 'Other' category all were with one occurrence.

Of all analyzed patients $48.3 \%$ had at least one reoperation due to an adverse event.

A total of 129 reoperations have been performed in the total patient group to address adverse events, averaging 0.87 reoperations per patient.

The most frequent surgical procedures were repositioning of the IPG because of pain, revision of the electrode because of suspected lead migration and reoperation for parameter change in patients implanted with the Itrell-I IPG. The 129 surgical procedures do not include replacement of the IPG for battery depletion.

19 revisions for battery depletion took place in 18 patients. One patient who was implanted in 1992 has had two revisions for battery depletion. The mean life span of the replaced IPG's in these patients was 73.7 months with a range between 28 to 127 months.

Chi-square correlation analysis between date of implantation, the sex of the patient, the type of complaints, follow-up time, the number of adverse events and whether the adverse events had been resolved at last follow-up and the result at last follow-up was performed.

The analysis showed a significant correlation between result and adverse events being resolved: $0.830(p=0.000)$. Also the surgery per year ratio and the final results were correlated: $0.397(p=0.000)$. 
However an inverse correlation was noted between result and the number of adverse events: $0.355(p=0.000)$. Patients without adverse events had the highest chance of a good result, but when patients had an adverse event, it was in most cases satisfactorily resolved.

The Chi-square test did not show a significant difference between the results of patients with or without a neurologic cause for their complaints.

Analysis of the data shows a clear decrease in the number of the adverse events and reoperations during the study period. Figure 1 shows the ratio of revisions and the ratio of adverse events in the patients implanted in each year divided by the number of implanted patients in that year.

Table 3. The number of implants, adverse events, reoperations and the ratios of revisions/implants and adverse events/implants for each year.

\begin{tabular}{lccccc}
\hline Year & \# Implants & \# Revisions & \# Adverse events & Revision ratio & Events ratio \\
\hline 1990 & 4 & 17 & 18 & 4.25 & 4.50 \\
1991 & 14 & 19 & 24 & 1.36 & 1.71 \\
1992 & 8 & 10 & 14 & 1.25 & 1.75 \\
1993 & 9 & 5 & 10 & 0.56 & 1.11 \\
1994 & 14 & 21 & 26 & 1.50 & 1.86 \\
1995 & 12 & 10 & 14 & 0.83 & 1.17 \\
1996 & 12 & 10 & 12 & 0.83 & 1.00 \\
1997 & 10 & 11 & 16 & 1.10 & 1.60 \\
1998 & 14 & 9 & 17 & 0.64 & 1.21 \\
1999 & 16 & 5 & 21 & 0.31 & 1.31 \\
2000 & 17 & 6 & 17 & 0.35 & 1.00 \\
2001 & 14 & 0 & 5 & 0.00 & 0.36 \\
2002 & 4 & 0 & 1 & 0.00 & 0.25 \\
2003 & 1 & 0 & 0 & 0.00 & 0.00 \\
\hline
\end{tabular}


Ratio of AE's and revisions divided by number of implants.

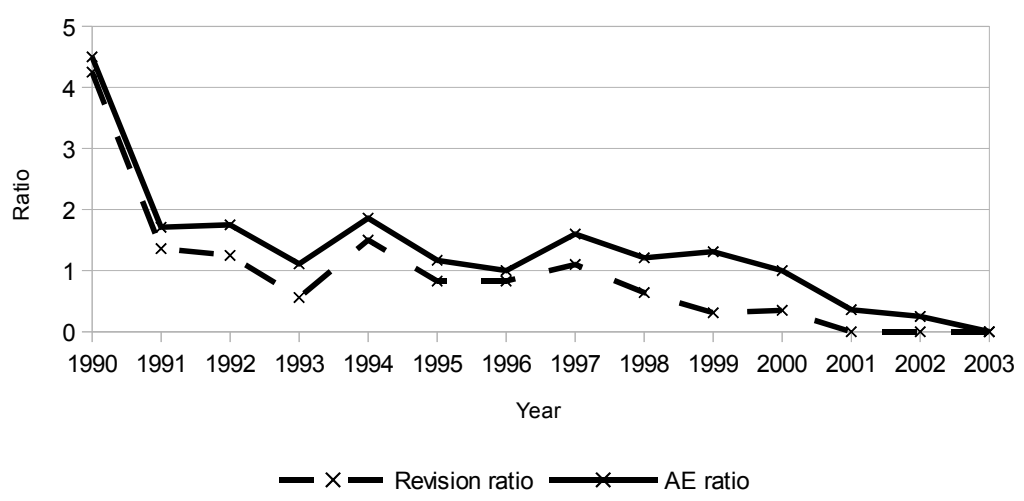

Figure 1. The ratio of revisions carried out in the patients implanted in each year divided by the number of the implants in each year. Revisions for empty batteries are excluded.

In the group of patients implanted before 1995 the mean number of reoperations was 1.56 , while in the group of patients implanted after 1995 the mean decreased to 0.49 reoperations. This difference is statistically significant $(p<0.0001)$.

The difference in the results between both groups changed as well: $60.7 \%$ of the patients implanted before 1995 had a good result while $65.9 \%$ of the patients implanted after 1995 had a good result. This difference is not statistically significant.

\section{Number of surgical revisions/patient before and after 1995}

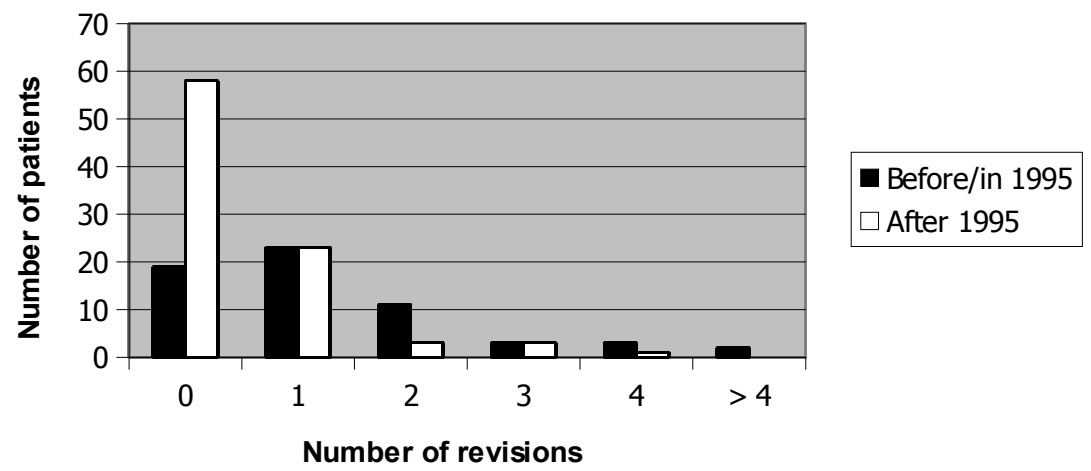

Figure 2. The amounts of patients with surgical revisions of their implanted system before / in 1995 and after 1995.

In $21(14.1 \%)$ of the 149 patients the neuromodulation system had been removed. 
The most common indication for explant was loss of efficacy (18 patients). The remaining three patients have been explanted due to uncorrectable side effects, one of these patients had an explant due to infection.

Of the explanted patients $15(71.4 \%)$ had urge symptoms, while 6 patients $(28.6 \%)$ suffered from retention. The mean follow-up was 53.6 months ( $s d=40.7$ ), while the follow-up ranged between 12 and 145 months. Statistical analysis in the 21 explanted patients shows a higher mean surgery/year ratio of $0.56(s d=0.09)$ than in the non-explanted group with a ratio of $0.12(s d=0.2)(p<0.005)$.

In 14 patients $(66.7 \%)$ the operation in which the IPG was explanted was their only reoperation, in the remaining explanted patients the number of surgical sessions before the explant ranged between 1 and 11 sessions.

\section{DISCUSSION}

Sacral neuromodulation gives lasting and satisfactory relief in patients with refractory symptoms of overactive bladder and urinary retention. ${ }^{4,7,14,15,16}$ Because the study setup is retrospective and voiding diaries were not always found in the file, the outcome of the patients was based on patient and physician satisfaction. Usually patients are satisfied when they are without symptoms or have very few symptoms. These results, however, are not comparable to other studies because of the subjective measurement of the outcome.

Some retrospective studies have been performed to identify predictive factors regarding the success of the Percutaneous Nerve Evaluation. ${ }^{17,18}$ So far no research has been done about predictive factors for the success of the definitive implant. In this study logistic regression analysis between result and date of implantation, sex of the patient, the type of complaints, follow-up time, the number of adverse events and whether the adverse events had been resolved at last follow-up has been carried out. However, none of these factors seemed to predict the outcome of neuromodulation. The therapy-dependent factors for a successful result that we identified were a low number of adverse events and surgical correction of an adverse event. There was no significant difference in the results between patients with complaints of neurogenic origin and idiopathic patients. The number of neurogenic patients (20 patients) was low in comparison with the non-neurogenic group (120 patients).

Previous studies by Weil and by Everaerdt show that psychiatric factors play a role in the first-year results of SNS. ${ }^{4,19}$ Weil et al. described that patients with a previous history of psychiatric disorder are more prone to lose effect of their implanted neuromodulation system, while Everaert et al. demonstrated a greater chance of dif- 
ferent outcomes between the temporary test and definitive implant in patients diagnosed with psychiatric disease. To avoid bias by placebo effect in patients, we established the criterion of a minimum follow-up of one year.

The rate of surgery for adverse events seems high, compared to other studies. In the literature the percentage of patients needing a reoperation is around $33 \%$, while in our study we found a reoperation rate of $48.3 \%$. ${ }^{4,5,7}$

This suggests a considerable learning curve in patient selection. Throughout the years we select patients more strictly. In the early years potential candidates underwent up to five PNE's when there was doubt if the patient reacted. Now patients get maximal two PNE's before offering them a two-stage tined lead procedure or excluding them from neuromodulation. Furthermore improved temporary lead design made the PNE more reliable. ${ }^{20}$

Another reason for higher reoperation rates is that the used equipment was still under development in the early years of SNS. Figure 1 shows that the greatest decrease in both adverse events and reoperations is between 1991 and 1992. An important reason for the decrease in adverse events and surgical interventions is the experience that is accumulated, particularly in the first years of the application of sacral neuromodulation. In 1994 both the Itrell-II and a new definitive electrode became available. Figure 1 shows that in 1994 the adverse events ratio and the reoperation ratio rises. This suggests a learning curve after the introduction of the new techniques.

The years thereafter the ratios drop, showing the benefits of the new techniques. The same phenomenon is seen in 1999 when the Interstim IPG is available and in 2000 when the buttock placement is introduced.

In 1997 Janknegt et al. tried to improve the testing procedure for neuromodulation by testing through the 2-stage procedure. ${ }^{13}$ The long-term efficacy of the 2-stage procedure has been determined by Scheepens et al. ${ }^{11}$ Recently the implant through the 2-stage procedure has been compared with the single-stage procedure in a randomized trial and seems to give a higher chance of a good result. ${ }^{21}$ Since 2002 a new procedure has been developed for lead implant. ${ }^{22}$ This procedure allows for minimal invasive lead placement under local anesthesia. The procedure can take place on outpatient basis and the tined lead can easily be removed. Therefore twostage testing through the tined lead procedure has become a valuable addition to the PNE. Experience shows that the rate of patients with positive screening results increases by using the tined lead. ${ }^{9}$ his is probably because the tined lead is less susceptible to lead migration and the tined lead allows for longer screening periods.

Since mid-2002 about 50 patients have been implanted with the tined lead procedure in our department. These patients are not included in this study.

We suspect that use of patients' sensory responses during the tined lead implant to guide lead placement gives less chance of failure of the definitive SNS system. Furthermore we expect a decrease in adverse events due to minimal invasive lead 
implant. These hypotheses have yet to be confirmed in a trial, which is currently ongoing in our clinic.

In 2000 buttock placement of the IPG has been investigated. ${ }^{10}$ The buttock placement reduced average operating time from 2.5 to 1.5 hours because turning of the patient during surgery was not necessary and the flank incision was not performed. More importantly, postoperative pain, postoperative infections and the need for reoperations for IPG position-related pain were reduced. Despite the lack of a randomized trial the buttock placement has quickly become the standard method of IPG implant

The occurrence of late failures remains still problematic in SNS. In this study 18 out of 149 patients have been explanted due to loss of efficacy. This group had more reoperations than the patients that were not explanted. No other predictors were found.

The cause of this phenomenon is still unknown, although we suspect that neuroplasticity of the micturition center could play an important role. Currently a trial is ongoing, investigating the effect of bilateral stimulation in patients with therapy failure in our department. Still, more research is needed in this particular group of patients.

\section{CONCLUSION}

Sacral neuromodulation produces prolonged subjective benefit in a group of patients with highly therapy resistant lower urinary tract symptoms. The decrease in the number of adverse events and reoperations over the study period suggests a considerable learning curve in the selection, the implantation and follow-up of patients with neuromodulation. Other contributing factors are the development and implementation of new surgical techniques and the new technical developments as the external patient programmer and new implant hardware.

\section{REFERENCES}

1. S. W. Siegel, Management of Voiding Dysfunction with an Implantable Neuroprosthesis. Urol Clin North Am 19, no. 1 (1992): 163-70.

2. H. E. Dijkema et al., Neuromodulation of Sacral Nerves for Incontinence and Voiding Dysfunctions. Clinical Results and Complications. Eur Urol 24, no. 1 (1993): 72-6.

3. J. L. Bosch and J. Groen, Sacral (S3) Segmental Nerve Stimulation as a Treatment for Urge Incontinence in Patients with Detrusor Instability: Results of Chronic Electrical Stimulation Using an Implantable Neural Prosthesis. J Urol 154, no. 2 Pt 1 (1995): 504-7.

4. E. H. Weil et al., Clinical Results of Sacral Neuromodulation for Chronic Voiding Dysfunction Using Unilateral Sacral Foramen Electrodes. World J Urol 16, no. 5 (1998): 313-21. 
5. S. W. Siegel et al., Long-term Results of a Multicenter Study on Sacral Nerve Stimulation for Treatment of Urinary Urge Incontinence, Urgency-frequency, and Retention. Urology 56, no. 6 Suppl 1 (2000): 87-91.

6. M M Hassouna et al., Sacral Neuromodulation in the Treatment of Urgency-frequency Symptoms: a Multicenter Study on Efficacy and Safety. The Journal of Urology 163, no. 6 (June 2000): 1849-1854.

7. R. A. Janknegt et al., Long-term Effectiveness of Sacral Nerve Stimulation for Refractory Urge Incontinence. Eur Urol 39, no. 1 (2001): 101-6.

8. U. Jonas et al., Efficacy of Sacral Nerve Stimulation for Urinary Retention: Results 18 Months After Implantation. J Urol 165, no. 1 (2001): 15-9.

9. M. Spinelli et al., New Percutaneous Technique of Sacral Nerve Stimulation Has High Initial Success Rate: Preliminary Results. Eur Urol 43, no. 1 (2003): 70-4.

10. W. A. Scheepens et al., Buttock Placement of the Implantable Pulse Generator: a New Implantation Technique for Sacral Neuromodulation--a Multicenter Study. Eur Urol 40, no. 4 (2001): 434-8.

11. W. A. Scheepens et al., Long-term Efficacy and Safety Results of the Two-stage Implantation Technique in Sacral Neuromodulation. BJU Int 90, no. 9 (2002): 840-5.

12. E. A. Tanagho and R. A. Schmidt, Bladder Pacemaker: Scientific Basis and Clinical Future. Urology 20, no. 6 (1982): 614-9.

13. R. A. Janknegt, E. H. Weil, and P. H. Eerdmans, Improving Neuromodulation Technique for Refractory Voiding Dysfunctions: Two-stage Implant. Urology 49, no. 3 (1997): 358-62.

14. M. Spinelli et al., Chronic Sacral Neuromodulation in Patients with Lower Urinary Tract Symptoms: Results from a National Register. J Urol 166, no. 2 (2001): 541-5.

15. Jonas et al., Efficacy of Sacral Nerve Stimulation for Urinary Retention: Results 18 Months After Implantation. J Urol. 2001 Jan;165(1):15-9.

16. E. H. Weil et al., Sacral Root Neuromodulation in the Treatment of Refractory Urinary Urge Incontinence: a Prospective Randomized Clinical Trial. Eur Urol 37, no. 2 (2000): 161-71.

17. E. L. Koldewijn et al., Predictors of Success with Neuromodulation in Lower Urinary Tract Dysfunction: Results of Trial Stimulation in 100 Patients. J Urol 152, no. 6 Pt 1 (1994): 2071-5.

18. W. A. Scheepens et al., Predictive Factors for Sacral Neuromodulation in Chronic Lower Urinary Tract Dysfunction. Urology 60, no. 4 (2002): 598-602.

19. K. Everaerdt, Patient Satisfaction and Complications Following Sacral Nerve Stimulation for Urinary Retention, Urge Incontinence and Perineal Pain: A Munticenter Evaluation. Int Urogynaecol J 11 (2000): 231-236.

20. E. H. Weil et al., Novel Test Lead Designs for Sacral Nerve Stimulation: Improved Passive Fixation in an Animal Model. J Urol 164, no. 2 (2000): 551-5.

21. K. Everaerdt, A Prospective Randomized Trial Comparing the 1-Stage with the 2-Stage Implantation of a Pulse Generator in Patients with Pelvic Floor Dysfunction Selected for Sacral Nerve Stimulation. European Urology 45 (2004): 649-654.

22. Spinelli et al., New Percutaneous Technique of Sacral Nerve Stimulation Has High Initial Success Rate: Preliminary Results. Eur Urol. 2003 Jan;43(1):70-4. 



\section{Chapter 5}

Medium Term Experience of Sacral Neuromodulation by tined lead implantation

A.C. van Voskuilen, D.J. A.J. Oerlemans, E.H.J. Weil, U. van den Hombergh and Ph.

E.V.A. van Kerrebroeck

BJU Int. 2007 Jan;99(1):107-10 


\section{ABSTRACT}

Objective: Describing patient selection and results of implant for sacral neuromodulation with the tined lead to median term follow-up.

Patients and methods: 49 patients, 39 with refractory overactive bladder symptoms, 10 with urinary retention, were implanted with the tined lead under local anesthesia. The average test period was 12.4 days, (sd 5.8 days). Patients were implanted when they had at least $50 \%$ improvement in voiding diaries. Average follow-up for implanted patients was 15.5 months ( $s d=7.9$ months). Change in voiding parameters was compared with the T-test.

Results: 10 patients had a single stage implant, 39 had a two-stage implant. Of 39 patients with 2-stage implant 31 (79.5\%) were positive, 8 (20.5\%) were not. 31 patients were included in follow-up. At last follow-up 28 (90.3\%) patients had more than $50 \%$ improvement in diary parameters and $3(9.7 \%)$ had not. In 21 patients with urgency symptoms the number of voids decreased from $11.7 \pm 8.9$ voids/day at baseline to $7.3 \pm 3.4$ voids $(p=0.1)$ Voided volume increased from $160.2 \pm 70.7$ $\mathrm{ml}$ to $231.1 \pm 119.5 \mathrm{ml}(\mathrm{p}=0.001)$. Leakages/day decreased from $9.5 \pm 8.7$ to $3.3 \pm$ $2.2(\mathrm{p}=0.17)$.

In 10 patients with retention the number of catheterisations decreased from $5.44 \pm$ 1.6 caths/day with a volume of $297.6 \pm 76.8 \mathrm{ml}$ to $1.2 \pm 1.7$ caths/day, volume was $111.6 \pm 158.1 \mathrm{ml}$. Mean number of voids increased from $3.7 \pm 3.8$ voids/day with a volume of $123.3 \pm 141.7 \mathrm{ml}$ to $4.2 \pm 2.4$ voids/day with a volume of $248.3 \pm 146.0$. There were no statistically significant differences in the parameters in the patients with retention.

Seven patients had an adverse event. One incomplete electrode migration was seen and was treated conservatively.

Conclusion: The new minimally invasive approach gives positive results on medium term. Two-stage testing with the tined lead seems to be more reliable than the classic PNE. The lead anchoring method seems sufficient for fixation of the electrode on median term. 


\section{INTRODUCTION}

Since its introduction about a decade ago sacral neuromodulation is applied in patients with refractory urge incontinence, urgency-frequency and non-obstructive urinary retention. ${ }^{1-5}$ Typically, potential candidates are tested with a temporary lead before the implantation of a definitive neuromodulation system is considered.

Clinical experience with the test procedure for neuromodulation showed that the temporary electrode is prone to migration during the subchronic test phase.

Due to this fact and due to the probability of bacterial infections in temporary electrodes that are implanted for a long period of time, the duration of the subchronic test phase is limited to a maximum of approximately one week. Moreover, there is a certain group of implanted patients who experience inconsistencies between the results of the test period and the results of the definitive implant.

In 2003 Spinelli et al. introduced a new minimally invasive technique for the implantation of a definitive electrode. ${ }^{6,7}$ By using this electrode the duration of the subchronic test phase can be prolonged and if the test results are insufficient, the electrode can easily be removed. In this article we describe our clinical results with this new minimally invasive procedure.

\section{METHODS}

49 patients with symptoms of overactive bladder or urinary retention, refractory to conservative therapy underwent an implant procedure using the new electrode. All patients had a Percutaneous Nerve Evaluation (PNE) with the conventional temporary electrode before the tined lead implant.

Under local anesthesia, in a minimally invasive procedure, a lead (Model 3889, Medtronic) was placed in the left or right S3 foramen. ${ }^{6}$ The lead is equipped with silicone tines that allow fixation in the tissue above the sacrum. Local anesthesia allowed for using both patients' sensory and motor responses as a guide. The depth of the electrode was controlled by lateral fluoroscopy.

The patients who had a positive result from the classic PNE had a single stage implant. In these patients the electrode was tunneled to an incision made at the ipsilateral gluteal area and connected to an extension cable.

This extension cable is connected to an implantable pulse generator (IPG, model 3023, Medtronic) which is inserted into a subcutaneous pocket in the gluteal area. The single stage procedure took on average 40 minutes. 
The patients with inconclusive results of the classic PNE underwent a two-stage procedure. In these patients the lead was tunneled to a small ipsilateral gluteal incision. After connection to an extension cable, the lead was tunneled to a contralateral exit point and connected to an external stimulator (model 3625, Medtronic).

The subchronic phase of the two-stage test took on average 12.4 days, (sd 5.8 days, 5-31 days). During this phase, patients were asked to keep voiding diaries to record the change in their urinary symptoms. These data were compared to baseline voiding diaries.

Patients with at least $50 \%$ improvement in their relevant urinary symptoms were implanted with an implantable pulse generator (IPG) as a second stage. In these patients the ipsilateral incision was reopened and the extension cable was disconnected and removed. A short extension cable was connected to the lead and an IPG was implanted. When the subchronic phase was not successful the lead and extension cable were disconnected and removed. The lead could easily be removed up to a month after implant by applying moderate traction. The first stage of the 2stage procedure takes on average 30 minutes, while the second stage was done in 15 minutes. All procedures were performed under local anesthesia on an outpatient basis.

After the implantation of the IPG, patients were followed on regular intervals of six weeks, six months and yearly thereafter.

Patients were instructed to contact the clinic when they had problems relating to the implanted system, All patients were instructed to avoid stretching, bowing and bicycling for at least 6 weeks after implant. After this period the patients were encouraged to resume all activities that they did before the implant. Each patient with loss of efficacy during follow-up had an X-ray to check whether there was electrode migration. In June 2005 all implanted patients have been asked to fill out another set of voiding diaries. These diaries have been compared with the baseline diaries. The parameters at baseline and at last follow-up have been compared using the Ttest (SPSS 12.0 For Windows, SPSS Inc, Chicago, USA.)

\section{RESULTS}

9 of the 49 patients were male, 40 were female. The mean age was 50.0 year ( $\mathrm{sd}=$ $13.2 \mathrm{yr}$, range $18-73 \mathrm{yr}$ ). 39 patients (79.6\%) were implanted for overactive bladder symptoms, 10 (20.4\%) had urinary retention. 10 patients had a positive classic PNE and were implanted in a single-stage procedure. The remaining 39 patients had an inconclusive PNE. They were re-tested in a two-stage procedure. Reasons for the 
inconclusive results were: discrepancy in recorded 24-hour output between the baseline and test period (13 patients), suspected electrode migration (7 patients) and technical failure of the PNE (2 patients). One patient with technical failure had a different anatomy due to spina bifida, in another patient the external stimulator switched off by itself. The other 17 patients with an inconclusive PNE had subjective improvement, while voiding diary analysis showed less than $50 \%$ improvement.

In the 39 patients who underwent 2-stage testing 31 (79.5\%) of the patients were eligible for the definitive implant and 8 (20.5\%) were not.

Of all 49 patients, 41 had a definitive implant either in a single stage or after twostage testing. 31 of them were included in further follow-up. 4 of the 10 patients who were not included had a follow-up of less than 6 weeks. A fifth patient with a follow-up of 18,3 months had loss of efficacy after a vaginal wall correction. A sixth patient died 8,9 months after implant due to a non-neuromodulation related cause and the seventh patient was explanted due to psychiatric reasons after 13.3 months follow-up, but had good results. This patient had hallucinations after implant and blamed adverse life events on having the nerve stimulation system.

Three patients were lost to follow-up and did not respond to repeated requests to fill in an additional diary. These ten patients have been excluded from analysis.

The mean follow-up of the 31 analyzed patients was 15.5 months, sd= 7.9 months. The follow-up ranged between 3.2 and 32.4 months. At last follow-up 28 (90.3\%) of the patients kept more than $50 \%$ improvement in at least one of the relevant voiding diary parameters and $3(9.7 \%)$ patients scored below $50 \%$ improvement. In all implanted patients the voided volume was increased from $153.0 \pm 86.9 \mathrm{ml}$ to 234.5 $\pm 122.5 \mathrm{ml}$ at last follow-up $(p=0.000)$.

In the patients with urge incontinence or urgency-frequency the mean number of voids at baseline was $11 ., 7 \pm 8.9$ voids/day. At last follow-up this decreased to $7.3 \pm$ 3.4 voids. ( $p=0.1$ ) The voided volume increased from $160.2 \pm 70.7 \mathrm{ml}$ to $231.1 \pm$ $119.5 \mathrm{ml}$ at last follow-up $(p=0.001)$.

The patients who had urge incontinence had on average $9.5 \pm 8.7$ incontinent episodes/day at baseline. At last follow-up this was $3.3 \pm 2.2$ episodes/day ( $p=$ $0.17)$.

In the 10 patients with retention the average number of catheterisations was at baseline $5.44 \pm 1.6$ caths/day and the catheterised volume was $297.6 \pm 76.8 \mathrm{ml}$. At last follow-up the mean number of catheterisations was $1.2 \pm 1.7$ caths/day and the catheterised volume was $111.6 \pm 158 ., 1 \mathrm{ml}$. Three of these ten patients had complete retention at baseline. At last follow-up 2 of these 3 patients had a normal voiding pattern. 
Six patients with retention do not catheterize any more, the remaining 4 patients catheterize up to a maximum of 2 times a day. The mean number of voids at baseline was $3.7 \pm 3.8$ voids/day with a volume of $123.3 \pm 141.7 \mathrm{ml}$. At last follow up the number of voids was $4.2 \pm 2.4$ voids/day with a volume of $248.3 \pm 146.0$. Analysis with the T-test showed that there were no statistically significant differences in the parameters in patients with retention

In 31 patients, 7 had a significant adverse event. Two patients had loss of efficacy after undergoing non-urological surgery. Both patients have positive results after up to 2 reprogramming sessions. Another patient with retention due to incomplete spinal cord lesion still has no benefit from his system. Two patients have had pain complaints at the IPG implant site. One had a reoperation under local anesthesia to reposition the IPG. The other patient had a buttock implant of the IPG, while she is dependent on a wheelchair. In this patient the IPG has been repositioned from the gluteal region to the abdominal wall. Both patients are free of pain and have positive results. A patient with positive results had an open revision because of troublesome leg stimulation. Investigation showed that the tined lead was placed too deep and stimulated the S2 nerve that is running below the level of the S3 root.

During follow-up one incomplete electrode migration has been noted. The patient presented at 5.3 months follow-up with different sensations and decreased efficacy after a fall. An X-ray of the sacrum showed that the electrode had moved about 2 to $3 \mathrm{~mm}$ in comparison to directly after the implant. The patient was successfully treated by reprogramming the stimulator.

\section{DISCUSSION}

Successful neuromodulation depends for the most part on careful selection of potential candidates. Until now the only means of selecting the patients with possible benefit was the classic PNE. The PNE however, has its limitations. At first the duration of the subchronic phase of the PNE is limited to a maximum of approximately one week because of the chance of infection.

Furthermore there is a chance of electrode migration. Edlund and coworkers mentioned in their study eight out of 20 patients with loss of efficacy during the subchronic phase of the PNE. ${ }^{8}$ The loss of efficacy is likely due to electrode migration, but not in all patients with an insufficient result electrode migration could be demonstrated by sacral X-ray

In 1997, the 2-stage approach was introduced. ${ }^{9,10}$ Testing with a definitive electrode allowed for longer testing periods and decreased the chance of a negative test due to electrode migration. Recently Everaert et al. proved in a randomized trail that 
testing with the 2-stage procedure gave more positive subjective results and more improvement in diary variables. ${ }^{11}$

As the tined lead can be implanted in a minimally invasive procedure under local anesthesia and can easily be removed, testing through the 2-stage procedure becomes easier and is applied more often.

The $79.5 \%$ of patients in this study who had a positive 2-stage procedure is higher than the $30 \%-62 \%$ of positive results with the classic PNE that was found in the literature. $^{8,11-14}$ This comparison is not entirely fair because a group of nonresponders have been filtered out by the classic PNE.

It does show that about $20 \%$ of the negative classic PNE's is false negative, possibly due to the fact that the testing period was too short to show clear results or maybe due to undetected electrode migration. In a recently published study Kessler et al showed a statistically significant difference between $50 \%$ positive responders after a period of 4 to 7 days compared to $80 \%$ after at least 14 days. ${ }^{15}$ These results indicate that the duration of the test period plays an important role in the selection of potential candidates for sacral neuromodulation. It would be interesting to compare the results of the PNE and the two-stage tined lead in a randomized prospective trial.

The patients with urgency show significant improvement at last follow-up compared to baseline. Although patient with retention improve, the parameters have not changed significantly. The group of retention patients is small and most patients had no complete retention at baseline.

The differences in number of voids and voided volume are relatively small, accounting for high $\mathrm{p}$ values. However the number of catheterizations has dropped to a mean of one catheterization per day and the need to catheterize is bothersome for the patients. $90 \%$ of the implanted patients keep a favorable result during the follow-up. This could suggest that, in addition to a better selection, placing the definitive lead implant under local anesthesia gives a higher chance of lasting positive results than the open implant.

Minimally invasive lead placement occurs under both sensory and motor responses, rather than only motor responses in the open procedure. We suspect that the use of sensory responses in addition to motor responses provides for better electrode placement and therefore less chance of complications and therapy failure. This has yet to be confirmed in a study with larger groups of patients and a longer follow-up period.

One patient had an incomplete electrode migration after a fall. We made sacral radiographies in each patient with loss of efficacy. Despite this rigorous screening policy, only one migration of about two to three $\mathrm{mm}$ was found, which could be 
treated conservatively. Kessler et al. described one patient with bilateral electrode migration, who was successfully treated by reprogramming. ${ }^{16}$

Two patients needed a reoperation due to pain at the IPG implant site.

One patient uses a wheelchair and the IPG implanted in the gluteal area caused discomfort due to the pressure of the wheelchair on the region of the implanted IPG. After repositioning the IPG in the abdominal wall, the patient is free of complaints. Therefore abdominal placement of the IPG should be considered in wheelchair bound patients. In the other patient with pain at the position of the IPG, we found during re-operation a subcutaneous nerve running through the pocket wall. After repositioning the IPG away from the nerve the patient was free of complaints.

\section{CONCLUSION}

The new minimally invasive approach for sacral neuromodulation is easy to perform compared to the classic open method. It gives positive results on both the short and medium term. Two-stage testing with the tined lead appears to be a more reliable testing method than the classic PNE because of a prolonged testing period and maybe due to a decreased risk of electrode migration. Therefore testing with a tined lead is a useful testing alternative in patients with an inconclusive result of the PNE. As there was only one lead migration during median term follow-up up to 3 years, it is obvious that that the lead anchoring method is sufficient for fixation of the electrode. However, long-term follow-up data on the tined lead performance and possible migration are not yet available. 


\section{REFERENCES}

1. Aboseif, S., K. Tamaddon, S. Chalfin, S. Freedman and J. Kaptein, Sacral neuromodulation as an effective treatment for refractory pelvic floor dysfunction. Urology, 2002. 60(1): 52-56.

2. Aboseif, S., K. Tamaddon, S. Chalfin, S. Freedman, M.S. Mourad, J.H. Chang and J.S. Kaptein, Sacral neuromodulation in functional urinary retention: an effective way to restore voiding. BJU international, 2002. 90(7): p. 662-665.

3. Weil, E.H., J.L. Ruiz Cerda, P.H. Eerdmans, R.A. Janknegt and P.E. Van Kerrebroeck, Clinical results of sacral neuromodulation for chronic voiding dysfunction using unilateral sacral foramen electrodes. World journal of urology, 1998. 16(5): 313-321.

4. Kerrebroeck, P.E.V., Van Voskuilen, A.C., PAS Study group. Long-term Results of InterStim Therapy for Voiding Disorders Demonstrate Sustained Efficacy and Acceptable Safety Profile. in European Association of Urology. 2004. Vienna.

5. Siegel, S.W., F. Catanzaro, H.E. Dijkema, M.M. Elhilali, C.J. Fowler, J.B. Gajewski, M.M. Hassouna, R.A. Janknegt, U. Jonas, P.E. van Kerrebroeck, A.A. Lycklama a Nijeholt, K.A. Oleson, and R.A. Schmidt, Long-term results of a multicenter study on sacral nerve stimulation for treatment of urinary urge incontinence, urgency-frequency, and retention. Urology, 2000. 56(6 Suppl 1): 87-91.

6. Spinelli, M., G. Giardiello, M. Gerber, A. Arduini, U. van den Hombergh and S. Malaguti, New sacral neuromodulation lead for percutaneous implantation using local anesthesia: description and first experience. Journal of Urology, 2003. 170(5):1905-1907.

7. Spinelli, M., G. Giardiello, A. Arduini and U. van den Hombergh, New percutaneous technique of sacral nerve stimulation has high initial success rate: preliminary results. European Urology, 2003. 43(1): 70-74.

8. Edlund, C., Hellstrom, M., Peeker, R., Fall, M., First Scandinavian Experience of Electrical Sacral Nerve Stimulation in the Treatment of the Overactive Bladder. Scandinavian Journal of Urology and $\mathrm{Ne}$ phrology., 2000. 34(6): 366-376.

9. Janknegt, R.A., E.H. Weil and P.H. Eerdmans, Improving neuromodulation technique for refractory voiding dysfunctions: two-stage implant. Urology, 1997. 49(3): 358-362.

10. Scheepens, W.A., G.A. Van Koeveringe, R.A. De Bie, E.H. Weil and P.E. Van Kerrebroeck, Long-term efficacy and safety results of the two-stage implantation technique in sacral neuromodulation. BJU International, 2002. 90(9): 840-845.

11. Everaerdt, K., Kerckhaerdt, W., Caluwaerdts, H., Audenaerdt, M., Vereecke, H., De Cuypere, G., Boelaerdt, A., Van den Hombergh, U., Oosterlinck, W., A Prospective Randomized Trial Comparing the 1Stage with the 2-Stage Implantation of a Pulse Generator in Patients with Pelvic Floor Dysfunction Selected for Sacral Nerve Stimulation. European Urology, 2004.45: 649-654.

12. Bosch, J.L. and J. Groen, Sacral nerve neuromodulation in the treatment of patients with refractory motor urge incontinence: long-term results of a prospective longitudinal study. Journal of Urology, 2000. 163(4): 1219-1222.

13. Hassouna, M.M., S.W. Siegel, A.A. Nyeholt, M.M. Elhilali, P.E. van Kerrebroeck, A.K. Das, J.B. Gajewski, R.A. Janknegt, D.A. Rivas, H. Dijkema, D.F. Milam, K.A. Oleson, and R.A. Schmidt, Sacral neuromodulation in the treatment of urgency-frequency symptoms: a multicenter study on efficacy and safety. Journal of Urology, 2000. 163(6): 1849-1854.

14. Weil, E.H., J.L. Ruiz Cerda, P.H. Eerdmans, R.A. Janknegt, B.L. Bemelmans and P.E. van Kerrebroeck, Sacral root neuromodulation in the treatment of refractory urinary urge incontinence: a prospective randomized clinical trial. European Urology, 2000. 37(2): 161-171.

15. Kessler, T.M., H. Madersbacher and G. Kiss, Prolonged sacral neuromodulation testing using permanent leads: a more reliable patient selection method? European Urology, 2005. 47(5): 660-665.

16. Kessler, T.M., Madersbacher, H. and Kiss, G., Bilateral Migration of Sacral Neuromodulation Tined Leads in a Thin Patient. Journal of Urology, 2005. 173: 153-154. 



\section{Chapter 6}

Is on-demand sacral neuromodulation in patients with OAB syndrome a feasible therapy regime?

Oerlemans DJ, van Voskuilen AC, Marcelissen T, Weil EH, de Bie RA, van Kerrebroeck PE.

Neurourol Urodyn. 2011 Nov;30(8):1493-6 


\section{ABSTRACT}

Sacral neuromodulation (SNM) of the lower urinary tract has proven to be safe and effective in patients with complaints of $O A B$ syndrome who are not responding to conservative therapy. After five years of treatment the implanted system is still effective in $56-71 \%$ of patients. The loss of effect could be caused by adaptation of the nerve system to prolonged stimulation of the sacral nerves. We set up a pilot intervention study. After a run-in period of two weeks all patients were randomized into two groups: one group with on-demand neuromodulation (intervention group) and one group with continuous neuromodulation (control group). For the remaining two weeks, the patients in the intervention group were instructed to switch their INS off by default and to switch it on again when they felt recurrence of symptoms. The patients in the control group were asked to use their system as normally. 10 out of 16 subjects reported a comparable symptom score during on-demand use of their neuromodulation system. Patients appreciated the comfort of being selfdeterment in the need for therapy. Possible benefits for patients could be: more autonomy, longer battery life of the implanted INS, decreasing the risk of adaptation by the nervous system.

\section{INTRODUCTION}

Sacral neuromodulation (SNM) of the lower urinary tract has proven to be safe and effective in patients with complaints of $O A B$ syndrome who are not responding to conservative therapy. ${ }^{1,2}$ SNM treatment is also used in patients with nonobstructive urinary retention and as an off-label treatment in patients with interstitial cystitis. ${ }^{3}$ After five years of treatment the implanted system is still effective in $56-71 \%$ of patients. ${ }^{4}$ The system consists of an implanted lead, stimulating the sacral nerves through one of the sacral foramina (preferably S3), connected to an implantable neurostimulator (INS) Typical battery life is 8-10 years; depending on the parameters used for stimulation. The expected lifespan of the recently introduced "Interstim II" lies around 3-5 years when used 24 hours/day.

Most of the patients with an implanted neuromodulation system for urinary complaints receive continuous neuromodulation 24 hours a day. However, they are instructed to turn the INS off at certain moments, for example when driving a car or working with heavy machinery. This is to prevent accidents caused by reactions to sudden changes in the electrical current.

Until now evidence is lacking whether it is necessary to apply 24 hours of neuromodulation per day or if discontinuation of neuromodulation is possible for a short period of time. 
In early trials on SNM the effect of prolonged discontinuation of neuromodulation for at least 72 hours showed return to baseline of voiding symptoms in all patients. This was reversible in most patients by turning the neurostimulator on again. ${ }^{1,5}$

The working mechanism of SNM is not fully understood, but seems to involve modulation of spinal cord reflexes and brain networks by peripheral afferents, rather than direct stimulation of the motor response of the detrusor muscle. SNM is proposed to activate or "reset" somatic afferent inputs that play a pivotal role in the modulation of sensory processing and micturition reflex pathways in the spinal cord. ${ }^{6,7}$ Furthermore, there are indications that SNM influences brain areas that are involved in detrusor hyperactivity, awareness of bladder filling, the urge to void and the timing of micturition. ${ }^{8,9}$

With on-demand rather than continuous neuromodulation, the physiological situation is more adequately approximated, since in the normal physiological situation there is no continuous tonic afferent information coming from afferents to the dorsal sacral horn. Therefore, patients are expected to have a lower risk of developing side effects of sacral neuromodulation such as gastrointestinal complaints, pain or menstrual cycle changes.

Clinical experience as well as the literature on SNM has shown that a certain percentage of implanted patients stop having benefit from their implanted system after a few years, despite adequate neuromodulation. A suggested hypothesis for this phenomenon is adaptation of the nerve system to prolonged stimulation of the sacral nerves. ${ }^{10}$ It could be expected that on-demand neuromodulation postpones or diminishes this effect.

The objective of this prospective pilot study was to investigate whether the ondemand use of SNM is a feasible therapy regime, therewith mimicking the normal physiological situation as well as diminishing the need for frequent replacement of the INS.

\section{PATIENTS AND METHODS}

This study was set up as a pilot intervention study. Patients who were treated with SNM for symptoms of urgency incontinence or urgency-frequency syndrome refractory to conservative therapy were included. Patients were considered eligible for the study when they had a functioning neuromodulation system with unchanged stimulation settings for at least six months. All participants were implanted with a system that includes a patient programmer, which gives the possibility to deactivate the system (Medtronic Interstim model 3023 and Model 3031A patient programmer, Medtronic, Minneapolis MN, USA). Patients with relevant co-morbidity were 
excluded (e.g. urinary tract infection, non-functioning system, malignancy of the urinary tract).

For this study a hybrid design was followed, based on previous work by Pocock. ${ }^{11}$ Instead of a 50:50 allocation of patients, we randomized $25 \%$ of the participants to control, permitting $75 \%$ to be assigned to the new intervention. We started with all patients and follow them for two weeks to establish a firm baseline (and the knowledge that with continuous SNM there will be maximum "on" time and very likely no change in symptoms). After two weeks patients were randomized to the on-demand group, while 5 stayed in the continuous (control) group. At the end of study, the data from the controls were compared to the baseline group and showed stability. This allowed us to combine all controls and compare them to the ondemand group. This enabled us to perform a transversal comparison with a minimum of patients. The main criteria for such an approach (rather recent included patients, the same procedure and investigator and identical outcome assessments) were all met in this trial.

All included patients signed an informed consent and were asked to register a voiding diary for two weeks (baseline) and to fill out a urologic measurement questionnaire (UMQ). This UMQ measures subjective urologic complaints in patients. This questionnaire is previously used in other studies regarding SNM for the measurement of subjective complaints. ${ }^{12}$ This UMQ consists of seven questions on urologic complaints; a score of 1 (much worse) to 5 (much better) can be given to each of the questions (Appendix 1). The questionnaire is previously used in other studies regarding SNM for the measurement of subjective complaints. ${ }^{12}$

This questionnaire was chosen because it has been used in our practice since the start of our neuromodulation database and appeared to be very useful in clinical practice and follow-up of individual patients. Although it is not a validated questionnaire, it gives a comparable scale of subjective complaints. We consider the data of interest to the clinician. as they give an impression of the magnitude of change.

The total study duration was four weeks. We started with a run in period of two weeks: in this period all subjects registered a voiding diary (baseline symptoms). At the end a urogical questionnaire had to be filled out. After the run-in period of two weeks all patients were randomized into two groups: one group with on-demand neuromodulation (intervention group) and one group with continuous neuromodulation (control group). For the remaining two weeks, the patients in the intervention group were instructed to switch their INS off by default and to switch it on again when they felt recurrent symptoms. So when they experienced more urgency symptoms or incontinence they turned their INS on. When symptoms had disappeared they could turn the INS off again. The patients in the control group were asked to use their system as normally: this means they were not allowed to 
change settings during the trial. At the end of this period, subjects were asked to fill out the UMQ again. (appendix 2)

With a voiding diary urinary complaints as well as the on and off hours were registered for both groups. The following voiding diary parameters were evaluated: number of voids/24h, voided volume/void, number of leakages/24h, number of pads/24h and the degree of urgency.

On-demand neuromodulation was considered successful when patients were able to switch the INS off for at least 4 hours per day, without worsening of urinary symptoms recorded with voiding diaries and UMQ. This cut-off point was set at 4 hours because this was considered a time span that could give a relevant change in battery lifespan.

Institutional review board approval was obtained for this study. All included patients signed an informed consent. Data analysis was performed using Statistical Package for the Social Science version 12.0 (SPSS Inc., Chicago IL, USA).

\section{RESULTS}

In total 26 patients were included in this study. Five patients were excluded from analysis because they did not fill out the voiding diaries correctly.

Complete voiding diaries were obtained of the remaining 21 patients, who were randomized into two groups. Five patients were randomized into the control group, 16 into the on-demand group. In the on-demand group there were 2 men and 14 women, in the control group only women. The mean age of these patients was 53.6 years. All patients had their neuromodulation system implanted between 1992 2004 for urgency incontinence. By the time they were participating in this study an Interstim model 3023 was implanted. In all patients the INS configuration was unchanged in the last half year and all subjects were capable of using their patient programmers correctly.

Baseline symptoms for both groups with continuous neuromodulation are shown in table 1 . There were no statistical differences in baseline parameters between the two groups.

Table 1. Baseline symptoms for both groups.

\begin{tabular}{lcccc}
\hline & Pads/day & Volume/void & \#micturition/day & \#leakages/day \\
\hline On Demand & 0.9 & $131 \mathrm{ml}$ & 8.7 & 2.9 \\
Control & 1.1 & $202 \mathrm{ml}$ & 7.3 & 2.8 \\
\hline
\end{tabular}

In the control group no differences were observed between the results of voiding diaries and UMQ during the first two weeks (baseline) and the second 2 weeks of this study. In the intervention group 10 of 16 subjects reported a comparable 
symptom score (no worsening) during on-demand use of their neuromodulation system. They had a mean off time of 12.4 hours a day.

Table 2. shows the mean off-times in hours a day for the intervention group.

Table 2. Number of patients with off-hours/day during intervention period.

\begin{tabular}{lccc}
\hline $\begin{array}{l}\text { Mean off-times in hours a day for the intervention group } \\
\text { Modulator off (hrs) }\end{array}$ & Worsening & No Worsening & Total \\
\hline $4-14$ & 0 & 2 & 2 \\
$14-24$ & 6 & 8 & 14 \\
Total & 6 & 10 & 16 \\
\hline
\end{tabular}

The six patients in the on-demand group that had worsening of symptoms were all patients who were symptom free during continuous use of their SNM system. With on-demand use of their system they experienced symptoms again. In these patients there was no difference in age.

Table 3 shows the different parameters for the subjects of the intervention group.

Table 3. micturition parameters during intervention period (mean off-time per day in hours).

\begin{tabular}{lcc}
\hline & $\begin{array}{c}\text { No. Pts. } \\
\text { (Not succesful) }\end{array}$ & $\begin{array}{c}\text { No Increase } \\
\text { (Succesful) }\end{array}$ \\
\hline Voids / $24 \mathrm{hr}$ & $4(10 \mathrm{~h})$ & $12(18 \mathrm{~h})$ \\
Leakage episodes & $6(8.4 \mathrm{~h})$ & $10(12.4 \mathrm{~h})$ \\
Pad use & $6(8.4 \mathrm{~h})$ & $10(12.4 \mathrm{~h})$ \\
Severity of leakage & $4(10 \mathrm{~h})$ & $12(18 \mathrm{~h})$ \\
\hline
\end{tabular}

After the intervention period all participating subjects filled out a Urologic Measurement Questionnaire (UMQ). With this questionnaire subjective differences between both periods were measured with seven questions. (min-max score 7-35). A score below 17 was considered as a worsening in subjective measurements. When subjects experienced no difference or improvement in all symptoms they should have scored 21 or higher.

Table 4. scores for subjective measurements (Urologic Measurement Questionnaire)

\begin{tabular}{lccc}
\hline Subjective change & Control & On-demand & Total \\
\hline Worsening $(</=17)$ & 0 & 8 & 8 \\
No Worsening $(>17)$ & 5 & 8 & 13 \\
Total & 5 & 16 & 21 \\
\hline
\end{tabular}




\section{DISCUSSION}

SNM is an approved treatment for different challenging urological conditions. ${ }^{1-3}$ Unfortunately some patients tend to loose their initial effect over the years. Long term multi-centre results show an efficacy off $56-71 \%$ for the different relevant parameters at five years. ${ }^{4}$ Possible explanations for failures during use of SNM include the potential placebo effect of test stimulation, insufficient test stimulation sensitivity before implantation and inadequate patient selection.

These explanations are supported by a good correlation between the one- and fiveyear success rates.

Adaptation of the nervous system, although never clearly studied, is another proposed mechanism for the loss of effect on the long term for prolonged stimulation of the sacral nerves. On-demand neuromodulation might be an ideal way of stimulation to prevent this phenomenon.

The results in table 1 shows that 14 out of 16 patients in the intervention group had their INS turned off for at least $14 \mathrm{hr}$ a day. 10 out of 16 patients could turn their INS off without worsening of complaints during this period, and 8 of these patients could do that for at least $14 \mathrm{hr}$.

In this study, $62.5 \%$ of the patients in the intervention group showed no increase in pad use and/or number of leakage episodes a day (Table 2). Looking to the number of voids in a day and the severity of leakage $75 \%$ of the intervention group had no increase in symptoms. This means that for an average group of patients treated with SNM for urgency incontinence almost $2 / 3$ could be treated with on-demand neuromodulation.

In the intervention group $50 \%$ of patients experienced worsening in subjective symptoms. This result can be explained by the way we instructed our patient. They were told to the turn the INS on during the intervention period when they felt recurring symptoms. As expected the patients in the control group experienced no difference (Table 3).

Another method of non-continuous neuromodulation is stimulation by cycling mode. This is possible with all implantable neurostimulators nowadays used for SNM therapy. With this setting the INS turns itself on and off in a preprogrammed cycle. For example the stimulation can be programmed on during 10 seconds and then off for 5 seconds.

Disadvantages of this method compared to on-demand neuromodulation is that a patient experiences this on/off event every time. Most of our patients reported discomfort during this cycling mode stimulation. A second disadvantage is that it is not the patient itself that determines the need for therapy. When we asked patients how they experienced on-demand neuromodulation, many patients appreciated the comfort of being self-determent in the need for therapy. They felt less dependent from their neuromodulation system. However, we did not measure 
the influence of this reported effect on quality of life. While an improvement in quality of life may be appreciated by some, those who are satisfied with continuous stimulation such that they no longer think about their prior voiding complaints, may view intermittent stimulation as an interruption and an impediment to their quality of life.

The long-term effects of on-demand neuromodulation were not studied in this pilot. Therefore long-term studies are necessary to determine if on-demand neuromodulation can prevent adaptation of the nervous system.

\section{CONCLUSION}

According to this pilot study, on-demand neuromodulation is a feasible therapy regime in patients with urgency incontinence successfully treated with SNM therapy. Possible benefits for patients could be:

- more autonomy; it allows patient to confidently turn of their SNM system without losing efficacy

- longer battery life of the implanted INS

- decreasing the chance of adaptation by the nervous system.

Nevertheless, larger, randomized controlled studies with longer follow-up are needed and should include quality of life measurements as well as a more detailed data on battery life improvement. 


\section{APPENDIX 1}

\section{Urologic Measurement Questionnaire}

1. Do you think that voiding in general goes better?

No, it's much worse / no it's worse / no difference / yes, some improvement / yes a lot better

2. Do you think that your flow has improved?

No, it's much worse / no it's worse / no difference / yes, some improvement / yes a lot better

3. Does your bladder feel emptier?

No, it feels much fuller / no, it feels a bit fuller / no difference / yes, it's emptier / yes, it's much emptier

4. Do you think you are drier?

No, much wetter / no, a bit wetter / no difference / yes, a bit drier / yes, much drier

5. Have your urge-sensations changed?

Clearly worsened / a bit worse / no difference / a bit less / clearly diminished

6. Has your voiding frequency changed?

No, I had to go more often / no, I had to go a bit more often / no difference / Yes, I had to go somewhat less often / Yes, I clearly had to go less often

7. Do you have more control over your bladder?

No, it's worse / No, it's somewhat worse / no difference / yes, it's better / yes, it's much better

Score $1-5$ 


\section{APPENDIX 2: COURSE AND TIMING OF THE STUDY}

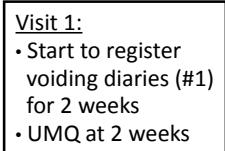

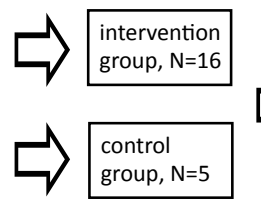

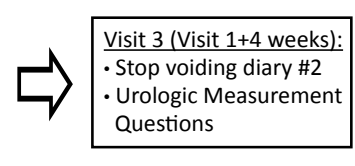

\section{REFERENCES}

1. Hassouna, M. M., Siegel, S. W., Nyeholt, A. A. et al.: Sacral neuromodulation in the treatment of urgency-frequency symptoms: a multicenter study on efficacy and safety. Journal of Urology, 163: 1849, 2000

2. Schmidt, R. A., Jonas, U., Oleson, K. A. et al.: Sacral nerve stimulation for treatment of refractory urinary urge incontinence. Sacral Nerve Stimulation Study Group. Journal of Urology, 162: 352, 1999

3. Jonas, U., Fowler, C. J., Chancellor, M. B. et al.: Efficacy of sacral nerve stimulation for urinary retention: results 18 months after implantation. Journal of Urology, 165: 15, 2001

4. van Kerrebroeck, P. E., van Voskuilen, A. C., Heesakkers, J. P. et al.: Results of sacral neuromodulation therapy for urinary voiding dysfunction: outcomes of a prospective, worldwide clinical study. Journal of Urology, 178: 2029, 2007

5. Weil, E. H., Ruiz-Cerda, J. L., Eerdmans, P. H. et al.: Sacral root neuromodulation in the treatment of refractory urinary urge incontinence: a prospective randomized clinical trial. European Urology, 37: 161,2000

6. Fowler, C. J., Swinn, M. J., Goodwin, R. J. et al.: Studies of the latency of pelvic floor contraction during peripheral nerve evaluation show that the muscle response is reflexly mediated. Journal of Urology, 163: 881, 2000

7. Vodusek, D. B., Light, J. K., Libby, J. M.: Detrusor inhibition induced by stimulation of pudendal nerve afferents. Neurourology and Urodynamics, 5: 381, 1986

8. Dasgupta, R., Critchley, H. D., Dolan, R. J. et al.: Changes in brain activity following sacral neuromodulation for urinary retention. Journal of Urology, 174: 2268, 2005

9. Blok, B. F., Groen, J., Bosch, J. L. et al.: Different brain effects during chronic and acute sacral neuromodulation in urge incontinent patients with implanted neurostimulators. BJU International, 2006

10. Agnew, W. F., McCreery, D. B., Yuen, T. G. et al.: Evolution and resolution of stimulation-induced axonal injury in peripheral nerve. Muscle Nerve, 22: 1393, 1999

11. Pocock, S.J., The combination of randomized and historical controls in clinical trials. Journal of chronic diseases, 1976. 29(3): p. 175-88.

12. Scheepens WA, de Bie RA, Weil EH, van Kerrebroeck PE. Unilateral versus bilateral sacral neuromodulation in patients with chronic voiding dysfunction. Journal of Urology. 2002 Nov;168(5):204650. 


\section{Chapter 7 \\ The Sexual Response in Patients treated With Sacral Neuromodulation for Lower Urinary Tract Symptoms or Faecal Incontinence}

A.C van Voskuilen, D.J. Oerlemans, N. Gielen, S.M.P. Lansen-Koch, E.H.J. Weil, J.J.D.M Van Lankveld, U. van den Hombergh, C.G.M.I. Baeten, P.E.V. Van Kerrebroeck

Urol Int. 2012 Apr 26 


\section{ABSTRACT}

Objectives: To determine whether SNM for urinary symptoms or faecal incontinence gives improvement of female sexual function and whether improvement is due to physiological factors or psychological factors.

Methods: Between 2002 and 2008, 8 patients have had an array of questionnaires before and after SNM implant. The questionnaires were: the Questionnaire for Screening for Sexual Dysfunctions, the Golombok Rust Inventory of Sexual Satisfaction, The Symptoms Check List-90, The Maudsley Marital Questionnaire and the McGill-Mah Orgasm Questionnaire. Three of these 8 patients underwent vaginal phletysmography before and after implant.

Results: no statistically significant changes have been found, although there seems to be a trend toward improvement in orgasm scores. In the phletysmography all three patients show increased Vaginal Pulse Amplitude with the stimulator turned on with both erotic and non-erotic stimuli.

Conclusions: This study does not show a clear effect of SNM on sexual function, although there seems improvement in orgasm scores. The lack of response on psychological questionnaires and the increase of VPA after SNM implant indicate that there might be a physiological response. 


\section{INTRODUCTION}

Since the 1990's sacral neuromodulation is used in patients with refractory idiopathic symptoms of overactive bladder $(O A B)$ or non-obstructive urinary retention. ${ }^{1,2,3}$ Recently its efficacy and safety is established in patients with faecal incontinence and constipation as well. ${ }^{4,5}$ Sacral neuromodulation (SNM) is carried out by electrical stimulation of one of both nerve roots running from both third sacral foramina.

During routine follow-up for SNM with the Interstim system in our outpatient clinic a number of female patients spontaneously reported improved sexual functioning in comparison to before their SNM implant. In the current literature, little is known about the relationship between SNM for urinary symptoms and possible impact on sexual functioning in female patients.

Sensory innervation of vagina and uterus is supplied through the pudendal nerve (external genitalia and clitoris), hypogastric nerve and pelvic nerve (both innervate the vagina and uterus). The pudendal nerve runs from the sacral plexus and contains fibers from S2, S3 and S4. The hypogastric and pelvic nerves contain sensory, sympathic and parasympathetic fibers. The pelvic nerve has fibers derived from S2, S3 and S4 as well. Moreover, the lumbosacral spinal cord contains interneurons that modulate the spinal sexual reflexes. ${ }^{6}$ A suggested hypothesis of the mechanism of action of SNM on lower urinary tract symptoms (LUTS) is modulating interneurons responsible for regulating the voiding reflex through stimulation of $\mathrm{C}$ afferent fibers. ${ }^{7}$ Based on the anatomical proximity and functional similarities of the sacral voiding and sexual nuclei, SNM might have a beneficial physiological effect on the sexual organs in female patients.

However, having lower urinary tract or bowel symptoms as described above has a marked negative influence on the quality of life of the patients, and successful SNM is known to increase quality of life. ${ }^{8}$ By feeling more comfortable, the interest in and the pleasure derived from sexual activities might increase in patients. Resolution of urinary or faecal symptoms can make these patients more comfortable and confident in their sexual relations.

This study is carried out to: 1 . Objectify whether SNM for LUTS or faecal incontinence does improve sexual function in female patients. 2. Determine whether improvement is due to a direct physiological response to SNM or because of improved well-being due to symptom resolution. 


\section{MATERIAL AND METHODS}

Permission for this study was obtained from the Medical Ethical Committee of the University Hospital Maastricht. Female patients aged between 18 and 60 years with urgency incontinence, urgency-frequency, non- obstructive urinary retention or faecal incontinence who were planned to undergo SNM implant with an Interstim system (Medtronic, Minneapolis, USA) were asked to participate in this study. Patients had to be sexually active with a partner and had to be able to read, comprehend and complete questionnaires written in Dutch. All participants had given written informed consent.

Patients with a known neurological cause for their voiding symptoms or with one of the other known contraindications for SNM, including obstructive voiding disorders, anatomical sacral defects, urinary tract infections, severe genitourinary prolapse, skin infections at the surgical site, malignancy of the urinary tract or anorectal malignancies, were excluded.

The subjects had either undergone a successful conventional Percutaneous Nerve Evaluation (PNE) test or were planned for a 2-stage procedure for their complaints. ${ }^{9,10}$ Patients who underwent the 2-stage procedure filled their baseline questionnaire in before the $1^{\text {st }}$ stage and were not included when the $1^{\text {st }}$ stage proved not successful. Therefore all included subjects had $50 \%$ or more improvement in relevant voiding or defaecation diary parameters during the trial phase and were eligible for a definitive SNM system implant. Voiding and defaecation diary analysis before and after implant of the SNM system has been performed.

All participants filled out an array of written psychological questionnaires before and after definitive implant of their SNM system, consisting of the Questionnaire for Screening for Sexual Dysfunctions (QSD; Vroege, 2001), the Golombok Rust Inventory of Sexual Satisfaction (GRISS), ${ }^{11,12}$ The Symptoms Check List-90 (SCL-90), ${ }^{13}$ The Maudsley Marital Questionnaire (MMQ) ${ }^{14,15}$ and the McGill-Mah Orgasm Questionnaire. ${ }^{16}$ All questionnaires were supplied in Dutch, native language of all subjects. The patients filled these lists in at home at their convenience. The questionnaires at baseline were accompanied with a small questionnaire on demographic data and specific sexual history. In the sexual history questions were asked about current complaints, past important sexual experiences, pregnancy and labour, and influence of the complaints on sexuality and relation with the partner.

The QSD questionnaire asks about several types of sexual dysfunction and is both physiologically and psychologically oriented. The chosen subdomains are Desire, Arousal, Orgams and Pain. In addition, a rating of the perceived impact of the dysfunction on subjects' functioning is given. Until now, there are no standard scores available of the QSD in the general population. 
The SCL-90 is an instrument for screening for psychopathology and is widely used in research to measure patient well-being after interventions. ${ }^{17}$

In the McGill-Mah Orgasm questionnaire patients are asked to rate words that might be applicable to their perception of orgasm on a 5-point scale. The subjects had a list of adjectives for orgasms, achieved through sex with a partner and orgasms realized by masturbation. The scores are divided in three main groups; Evaluative, Affective and Sensory. In the Evaluative domain all cognitions of orgasm are summarized, while Affective is the domain for the emotional aspects of orgasm. The Sensory domain describes the somatic aspects of orgasm.

The Maudsley Marital Questionnaire measures marital dissatisfaction.

The Golombok and Rust Inventory of Sexual Satisfaction questionnaire measures sexual dysfunction and has subscales dealing with physiological effects and emotional effects such as avoidance behaviour.

The initial study protocol described measurements of the Vaginal Pulse Amplitude through vaginal plethysmography. ${ }^{18,19,20}$ Patients were given visual sexual and neutral stimuli in a visually enclosed environment, while through vaginal plethysmography the Vaginal Pulse Amplitude (VPA) was measured. A total of five erotic video fragments with audio, each lasting 330 seconds, were shown to each patient in two measurement sessions. The fragments were from a female-centered erotic movie and randomly alternated with neutral, ie. non-erotic videos. Two video fragments were shown at the measurement session before SNM implant, three fragments in the measurement session after SNM implant. The patients were asked to express levels of subjective sexual excitement during the stimuli by turning a dial, calibrated to a 100-point scale. In the measurement session after implantation measurements were made with the SNM system switched on and off. The off-measurement was made 20 minutes after switching the SNM system off.

In contrast with the Vaginal Blood Volume (VBV), the VPA is indicative for rapid changes in vaginal vasocongestion and regarded as a more sensitive and specific index for physiological sexual arousal. ${ }^{21}$ Serum progesteron, 17- B-oestradiol and testosteron was measured to determine in which phase of the menstrual cycle the subjects were in at time of measurement.

The plethysmograpic data was amplified and analyzed with a personal computer. The VPA was defined as the mean and maximum difference between the vaginally measured heart pulse amplitude in a sample block. The mean VPA was calculated over segments of 30 seconds. The first 60 seconds after start of a stimulus were considered a run-in period and were not analyzed. The highest VPA during the 30 second block was designated as the maximum VPA. The acquired data was visually inspected for movement artifacts, which were excluded from further analysis.

The difference between the mean and the maximum VPA at baseline and with the SNM system implanted were calculated. 
Several subjects withdrew their informed consent when being confronted with the measurement setup and inclusion of study subjects was very slow.

Therefore the plethysmography protocol was abandoned after three patients.

Statistical analysis was performed with SPSS 17.0 (SPSS, Inc., Chicago, II.) Differences between scores before and after SNM were tested for statistical significance with the Mann-Whitney test. Analysis of the data showed that the data was not normally distributed. Data were described as median and range, considering the small amount of study subjects.

\section{RESULTS}

A total of 10 patients were included between January 2002 and December 2008 and had a successful PNE or 2-stage procedure. One patient filled in the questionnaires at baseline, but was explanted due to infection before the second set of questionnaires could be administered. She was excluded from the study. Another patient refused further participation in the study after the baseline questionnaires were taken and was excluded from analysis as well. Eight patients were ultimately analyzed.

Median age of these 8 patients was 53.5 years (range 21-61). Six patients had urinary urgency symptoms, one patient suffered from urinary retention and one patient had faecal incontinence. Median duration of their complaints was 6.5 years (range 2.0 - 36.0). All patients had satisfactory results of their definitive SNM implant.

All patients were heterosexually oriented and were sexually active during the study period. All patients but one had a steady relationship. The partner of one patient had suffered from a stroke before patient enrolled in this study, but the couple was still sexually active. One patient reported an unpleasant sexual experience in her past. Six patients were postmenopausal. One patient had a Wertheim-Meigs procedure because of cervical carcinoma. When asked 'do you have any current sexual complaints?' on the history form at baseline, only one of the patients answered affirmative.

The results at baseline and post implant of the Questionnaire for screening Sexual Dysfunctions (QSD) are shown in table 1. The ranges between lowest and highest scores are high, signifying large differences in QSD item scores between subjects in this small group. There is a small increase in the occurrence of genital pain after SNM implant. 
The median of 0 with a range of 9.5 is explained by the fact that five patients out of eight report no genital pain and three patients do with scores of 1.0, 5.0 and 9.0. There is no difference in the orgasm score before and after SNM, but the decrease in range indicates that in patients reporting high dysfunction scores before SNM the dysfunction decreases, therefore approaching the patients who already reported little dysfunction. The sum score of the QSD shows a small overall deterioration in the group of patients, but the range increases as well. Because of the small sample size no statistical significance could be found.

Table 1. The results of the Questionnaire for screening Sexual Dysfunctions (QSD).

\begin{tabular}{|c|c|c|c|c|c|}
\hline \multicolumn{6}{|c|}{ Results of the QSD before and after SNM implant } \\
\hline & \multicolumn{2}{|c|}{ Baseline } & \multicolumn{3}{|c|}{ Post SNM implant } \\
\hline & Median & Range & Median & Range & $\mathrm{p}$ \\
\hline Desire & 5.10 & 8.80 & 4.85 & 8.80 & 0.944 \\
\hline Arousal & 3.00 & 28.00 & 3.67 & 28.00 & 0.959 \\
\hline Orgasm & 2.33 & 12.67 & 2.33 & 9.67 & 0.645 \\
\hline Pain & 0.00 & 9.50 & 0.50 & 9.00 & 0.878 \\
\hline Total & 11.42 & 7.53 & 13.65 & 55.47 & 0.959 \\
\hline
\end{tabular}

Median scores and range (difference between the highest and the lowest score) of baseline (before SNM implant) and post-implant are shown. The p-values are shown as well. A higher score means more dysfunction

Table 2 shows scores on the SCL-90 before and after SNS implant. Anxiety, depression, and problems in interpersonal sensitivity were decreased after implant, but the differences were not statistically significant. Agoraphobia and somatic problems remained equal. In agoraphobia the range decreased by four points while in somatic problems the range increased by five points. The decrease in the range of the agoraphobia score is caused by a decrease in patients who scored relatively high at baseline. In addition, the score in patients with low agoraphobia scores is considered a floor effect. A small increase in a low agoraphobia score will not be measured by the SCL-90. The increase in range in the somatic problem score is explained by an outlier scoring 22 points. The SCL-90 total score is slightly decreased. Considering the overall low mean level of psychopathology, the small change effects might be considered a floor effect. 
Table 2. SCL-90 scores before and after SNM implant.

\begin{tabular}{lrrrrr}
\hline \multicolumn{2}{l}{ Results of the Symptom Check List-90 before and after SNM } & \multicolumn{3}{c}{ Post SNM implant } \\
& Median & Range & Median & Range & $p$ \\
\hline Anxiety & 12.50 & 9.00 & 11.00 & 8.00 & 0.279 \\
Agoraphobia & 7.00 & 7.00 & 7.00 & 3.00 & 0.959 \\
Depression & 20.00 & 26.00 & 18.50 & 23.00 & 0.878 \\
Somatic problems & 14.50 & 18.00 & 14.50 & 23.00 & 0.959 \\
Insufficiency & 10.50 & 14.00 & 11.00 & 13.00 & 1.000 \\
Interpersonal sensitivity & 23.00 & 20.00 & 20.50 & 13.00 & 0.721 \\
Hostility & 6.50 & 2.00 & 6.00 & 3.00 & 0.328 \\
Sleeping problems & 4.50 & 7.00 & 4.00 & 8.00 & 0.959 \\
SCL-90 total & 109.50 & 105.00 & 102.50 & 79.00 & 0.798 \\
\hline
\end{tabular}

Median scores of the subscales of the SCL-90 before and after SNM implant and p-scores are shown. Higher scores reflect more symptom perception.

Analysis of the scores of the Maudsley Marital Questionnaire shows no changes on dissatisfaction in all three items (relational dissatisfaction, sexual dissatisfaction and dissatisfaction in general life.) Therefore the data are not shown in a table.

The results of the McGill Mah Orgasm Questionnaire are shown in table 3. One patient stated that she does not masturbate, therefore she is excluded from analysis of the McGill Mah questionnaires. A trend towards improved orgasmic appreciation in nearly all domains, both with partner as orgasm achieved through masturbation is implicated by the findings, but no significant $p$-values have been measured. The sensitive with partner domain is decreased with one point.

Table 3. The results of the McGill Mah Orgasm Questionnaire for sex with a partner and for masturbation before and after SNM implant in seven patients.

\begin{tabular}{|c|c|c|c|c|c|c|}
\hline \multicolumn{7}{|c|}{ Results of the McGill Mah Orgasm Score before and after SNM } \\
\hline & & \multicolumn{2}{|c|}{ Baseline } & \multicolumn{3}{|c|}{ Post SNM } \\
\hline & & Median & Range & Median & Range & $\mathrm{p}$ \\
\hline \multirow[t]{3}{*}{ Masturbation } & Sensitive & 18.0 & 37.0 & 23.0 & 26.0 & 0.383 \\
\hline & Affective & 19.0 & 31.0 & 23.0 & 26.0 & 0.456 \\
\hline & Evaluative & 15.0 & 21.0 & 19.0 & 18.0 & 0.383 \\
\hline \multirow[t]{3}{*}{ Partner } & Sensitive & 42.0 & 60.0 & 41.0 & 43.0 & 0.805 \\
\hline & Affective & 36.0 & 41.0 & 38.0 & 24.0 & 0.902 \\
\hline & Evaluative & 24.0 & 22.0 & 25.0 & 11.0 & 0.8057 \\
\hline
\end{tabular}

Table 4 shows the scores regarding sexual dysfunction and dissatisfaction (GRISS) before and after SNM. Improvement with regard to sexual functioning appears to be reported on some subcategories, namely infrequency and dissatisfaction. 
The other scores do not seem to change. The total score seems to improve. Statistical analysis shows no statistical significance in these differences.

Table 4. Results of the Golombok and Rust Inventory of Sexual Satisfaction.

\begin{tabular}{lrrrrr}
\hline \multicolumn{2}{l}{$\begin{array}{l}\text { Results of the GRISS before and after SNM } \\
\text { Baseline } \\
\text { Median }\end{array}$} & \multicolumn{1}{c}{ Range } & $\begin{array}{c}\text { Post SNM } \\
\text { Median }\end{array}$ & Range & $\mathrm{p}$ \\
\hline Infrequency & 7.50 & 8.00 & 5.50 & 7.00 & 0.328 \\
Noncommunication & 4.00 & 6.00 & 4.50 & 5.00 & 0.878 \\
Sexual Dissatisfaction & 7.50 & 12.00 & 6.00 & 13.00 & 0.574 \\
Avoidance & 5.00 & 10.00 & 5.00 & 10.00 & 1.000 \\
Nonsensibility & 5.00 & 14.00 & 5.00 & 10.00 & 0.798 \\
Vaginismus & 4.50 & 13.00 & 4.50 & 14.00 & 0.878 \\
Anorgasmia & 9.50 & 13.00 & 9.50 & 11.00 & 0.959 \\
Griss Total & 52.50 & 74.00 & 51.00 & 76.00 & 0.878 \\
\hline
\end{tabular}

Median scores, range and p-values of baseline and post SNM-implant are shown. A higher score signifies more dysfunction.

Three patients underwent measurement with vaginal plethysmography before and after SNM implant. One patient (61 years old) was suffering from urgency incontinence, one (60) had faecal incontinence and one (21) had urinary retention. Graphic 1 shows the mean VPA of the measurements in these three patients. There is no statistical significance in the changes in VPA in these three patients. There is, however, a trend towards increased mean VPA response after SNM implant. Even when the subjects are watching a neutral video or when the stimulator is off during an erotic video, mean VPA is higher than before SNM. This might imply an enduring effect of SNM on pelvic vasocongestion, that is not dependent on sexual stimuli. The subjective measurement of sexual arousal, measured with a calibrated dial, remained low in all three patients and did not change after SNM implant. 
Average Vaginal Pulse Amplitude with visual stimuli before and with SNM implant

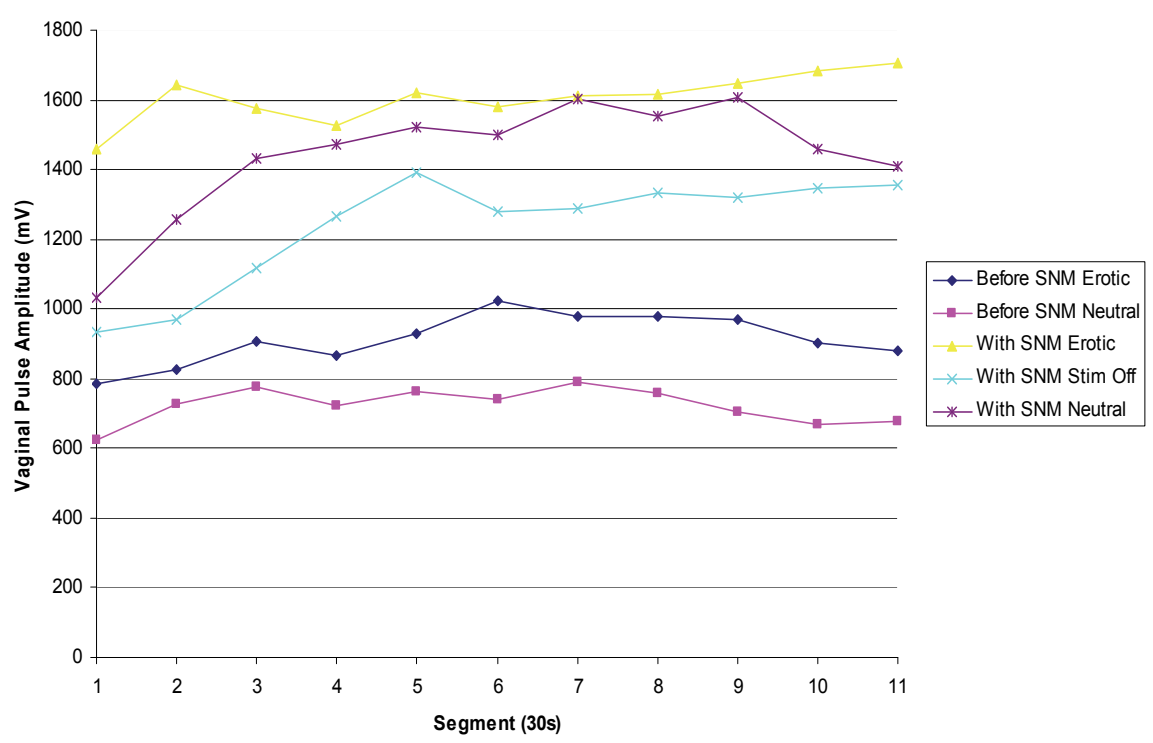

Figure 1. Mean VPA measurements with erotic and neutral visual stimuli of the three subjects before and after SNM implant. The changes are not statistically significant.

\section{DISCUSSION}

This study was initiated because at routine follow up visits of patients with SNM several female patients spontaneously reported improvement in their sexual functioning. The results of this study do not clearly support the improvement mentioned by several of our patients.

However, there are several other studies on the influence of SNM on the female sexual function where significant positive results were found.

Pauls et al. reported improvement in the FSFI (Female Sexual Function Index) score in eleven female patients with a SNM implant for LUTS. ${ }^{22}$ Significant improvement with regard to sexual desire was found in all patients in that study. There was a significant difference in seven of those eleven patients with regard to lubrication, orgasm and pain. Interestingly, all patients in Pauls' study improved on the FSFI, while only three subjects reported subjective improvement of sexual functioning. In 2008 Lombardi et al. presented the results of their study on the effect of sacral neuromodulation on sexuality in 31 female patients. In this study both patients with idiopathic and with neurological causes for their LUTS were included. Improvement in FDSF (Female Sexual Distress Scale) and FSFI after SNM was found in both patient groups. $^{23}$ 
Another study on the effects of SNM for LUTS on female sexual function was carried out by Zahibi et al. ${ }^{24}$ Characteristics of this study were: a large proportion of the patients had pelvic pain as well as LUTS. The study group chose to perform bilateral SNM and to position the electrodes epidural in the sacral canal, thus stimulating the nerves of S2 and S4 as well as S3.

A significant improvement in the FSFI was found. Domains with no significant improvement were: 'desire' and 'pain'.

Ingber et al used the FSFI questionnaire on 54 female patients who were scheduled to receive an neuromodulation implant. They administered the FSFI before neuromodulation implant and six months after implant and found non-significant improvements in female sexual function after implant. ${ }^{25}$ Only half of their analyzed population was sexually active. Both neuromodulation by sacral neuromodulation and by pudendal implant techniques was used in their study. ${ }^{26}$

In one study, published in 2009, Jarrett et al. administered a self-written sexual questionnaire to patients with fecal incontinence before and after their SNM implant. In 9 sexually active patients (of 16 patients) an average of 36,7\% improvement (range $0-100 \%$ ) in reported sexual functioning. ${ }^{27}$

The increase in pain scores in the QDS in this study might be explained by the fact that these patients are measured at six weeks after the implant and are not experienced yet with adjusting the stimulation amplitude with the remote control that each patient receives after implant on an SNM system.

In the study by Pauls et al. subjective improvement is seen in all the stages of the sexual response cycle except for arousal.

Sexual arousal is a response to a sexually attractive stimulus and has both a physiological and a subjective component. Many people define arousal as the feeling of sexual attraction, which in the sexological literature is referred to by the term desire. The results of our study show a trend towards improvement in desire to engage in sexual activity after SNM. In this article we define sexual arousal as the genital and subjective responses to erotic stimulation when engaging a sexual encounter. In this study a trend towards deterioration of subjective arousal is found in the QSD, but improvement of vaginal vasocongestion with SNM was measured, regardless of the kind of stimulus. Vaginal vasocongestion is one of the main genital components of sexual arousal in female patients.

In the literature is evidence that the concordance between physiological genital arousal and the perceived arousal by females is low, compared to the concordance in men. ${ }^{28,29}$ The reason for this phenomenon is still unclear. A suggested hypothesis is that genital arousal in men, i.e. erection, gives more sensory and visual feedback to the male than the genital response does in the female.

In the three patients measured with vaginal phletysmography our study we found clues for a discrepancy between physiological improvement and psychological dete- 
rioration of sexual arousal. In contrast, Pauls et al. ${ }^{22}$ found improvement in arousal in their study. An explanation of this discrepancy might be that SNM improves vaginal vasocongestion during the arousal phase, but this is not perceived by the women. This might explain why Pauls et al. did not find improvement in their questionnaire-based study. In the current study no improvement was reported in the questionnaire part.

The SCL-90 shows a nonsignificant decrease in symptom perception. Interpersonal sensitivity is the symptom that shows the most reaction to SNM. The improvement in interpersonal sensitivity might be explained by the decrease of worries about hygienic problems and incontinence pad use. As a result of this patients can interact with other persons more freely. The decrease in anxiety and hostility can be considered as confirming this hypothesis. The observed absence of further improvement with regard to psychopathology may be due to the low baseline level of these problems.

Most convincing is the trend towards improvement in the McGill-Mah Orgasm scores. The perception of patient's orgasm both with and without partner increases in all three domains. In the QSD orgasm scores remain equal between the two measurements The QSD is a diagnostic tool for perceived dysfunction and therefore might not adequately measure improvement in patients with little or no dysfunction, while the McGill Mah score might be more sensitive for these effects.

There are case reports on female patients who receive lumbal epidural electrostimulation for non-sexual complaints, who have spontaneous orgasms when the stimulator is turned on. ${ }^{30}$ Patients with a definitive SNM system implant have a remote control, enabling them to adjust stimulation amplitude. Until recently patients were advised to set stimulation amplitude that the stimulation is felt, but not in an unpleasant way. Most female patients feel the stimulation in the genital area. As far as we know, there have never been reports of patients having spontaneous orgasms while receiving SNM therapy or while undergoing an acute PNE or twostage procedure. A recent publication by Baeten et al. shows that SNM has an effect on uterus contractility. ${ }^{31} A$ decrease of frequency of contractions is seen with the SNM system switched on. But as the measurements have been performed in patients in resting state, it is not clear what the effect is of SNM on the uterus while the patient has an orgasm.

The three patients who underwent plethysmographic measurements with erotic and neutral stimuli before and after SNM all show a (non-significant) increase in VPA after SNM implant. They do not report a difference in subjective arousal while watching the videos before and after SNM. However, they do show increased pelvic blood flow in the plethysmographic measurements. This trend towards increased 
mean VPA is present, influenced by neither the kind of stimulus (erotic or neutral) nor the on or off-modus of the stimulator. SNM might have a general enhancing physiological effect on pelvic blood flow, and therefore might improve sexual functioning.

In the current literature on SNM there is no evidence found for the effect on SNM on pelvic blood flow. The increase in pelvic vasodilatation may be caused by stimulation of parasympathetic fibers by SNM.

When looking at the data of the questionnaires and the discrepancy between objectively measured VPA and subjective arousal in this prospective study and at the available literature on SNM and female sexual functioning, there are clues that SNM may give an improvement in sexual function in female patients. This improvement seems to be mostly because of a psychophysiological effect and for a lesser part due to an improved well-being.

None of the results in this study was statistically significant. The most obvious cause for non-significance is the low number of patients tested. Recruitment of study subjects was very slow. Several study subjects withdrew their consent because of the plethysmography. To give an impulse to recruitment, the plethysmography protocol was abandoned. After that recruitment still was slow. A reason for this might be that many patients with prolonged LUTS or fecal symptoms are not sexually active (any more) because of their complaints, while an inclusion criterion of this study was current sexual activity. Therefore a selection bias might have been introduced, that could not have been avoided, since non-sexual active patients could not participate in the measurements. In the study by Ingber et al, where sexual activity was not an inclusion criterion, half of the patients were not sexually active when analyzed. Moreover, a large proportion of the group of patients that have urinary or fecal symptomatoloy and are considered for SNM therapy are postmenopausal patients. In this group considerable co-morbidity can be found such as hysterectomy, vascular disease, diabetes or arthrosis that might also preclude patients from engaging in sexual activity. Furthermore, our results show relatively low levels of sexual pathology, and the ceiling effects or floor effects might not be measured with our questionnaires. In their article Pauls et al. reported slow subject recruitment as well.

Another reason for non-significance is that our population is heterogeneous. Three different diagnostic categories have been included, namely, urgency incontinence, urinary retention and faecal incontinence. Furthermore the sample has been heterogeneous with regard to pre- or postmenopausal status, co-morbidity, and coping strategies.

Nevertheless, this is the first prospective study in which vaginal plethysmography was used to investigate the nature of the improvement in sexual function in female patients with SNM. 
Further research into this interesting field would ideally be a multicentre study to accumulate sufficient patients for statistical analysis and even subgroup analysis. Also, a study which compares the prevalence of sexual dysfunction within these patient groups to age-matched patients without LUTS or faecal incontinence could be very interesting. Research might also be directed into the comparison of improvement of sexual function between patients with SNM versus other therapies, for example intravesical botulin toxin injection, to differentiate between a direct physiologic mechanism of SNM versus a psychological action because of decrease of symptoms on sexual functioning. Another research project would be investigating whether SNM does indeed increase pelvic blood flow and therefore has a physiological effect on sexual functioning. Also the occurrence of improvement of sexual function in male patients with SNM could be studied, as males show more concordance between physiological versus psychological arousal. However, in our patient population we have no reports of male patients with improvement of sexual functioning after SNM.

\section{CONCLUSION}

The results of this study suggest there might be sexual improvement in female patients receiving SNM therapy for urgency symptoms, urinary retention or faecal incontinence. This improvement seems to be more physiologically than psychologically mediated.

\section{REFERENCES}

1. Philip E V van Kerrebroeck et al, Results of sacral neuromodulation therapy for urinary voiding dysfunction: outcomes of a prospective, worldwide clinical study. Journal of Urology 178, no. 5 (November 2007): 2029-2034.

2. M. M. Hassouna e.a., Sacral neuromodulation in the treatment of urgency-frequency symptoms: a multicenter study on efficacy and safety. Journal of Urology 163, no. 6 (2000): 1849-1854.

3. H. S. Shaker en M. Hassouna, Sacral nerve root neuromodulation: an effective treatment for refractory urge incontinence. Journal of Urology 159, no. 5 (1998): 1516-159.

4. J Melenhorst e.a., Sacral neuromodulation in patients with faecal incontinence: results of the first 100 permanent implantations. Colorectal Disease: The Official Journal of the Association of Coloproctology of Great Britain and Ireland 9, no. 8 (October 2007): 725-730.

5. M. A. Kamm e.a., Sacral nerve stimulation for intractable constipation. Gut 59, nr. 3 (March 2010): 333-340.

6. K E McKenna, The neurophysiology of female sexual function. World Journal of Urology 20, no. 2 (Juni 2002): 93-100.

7. H. Shaker, Role of C-afferent Fibres in the Mechanism of Action in Chronic Spinal Cord Injury. BJU international 85 (2000): 905-910. 
8. A. K. Das, A. M. Carlson, en M. Hull, Improvement in depression and health-related quality of life after sacral nerve stimulation therapy for treatment of voiding dysfunction. Urology 64, no. 1 (2004): $62-8$.

9. A C Van Voskuilen e.a., Medium-term experience of sacral neuromodulation by tined lead implantation. BJU International 99, no. 1 (January 2007): 107-110.

10. M. Spinelli e.a., New sacral neuromodulation lead for percutaneous implantation using local anesthesia: description and first experience. J Urol 170, no. 5 (2003): 1905-1907.

11. J Rust en S Golombok, The Golombok-Rust Inventory of Sexual Satisfaction (GRISS). The British Journal of Clinical Psychology / the British Psychological Society 24 ( Pt 1) (February 1985): 63-64.

12. $M$ M ter Kuile e.a., The Golombok Rust Inventory of Sexual Satisfaction (GRISS): psychometric properties within a Dutch population, Journal of Sex \& Marital Therapy 25, no. 1: 59-71.

13. L R Derogatis, R S Lipman, en L Covi, SCL-90: an outpatient psychiatric rating scale--preliminary report. Psychopharmacology Bulletin 9, no. 1 (January 1973): 13-28.

14. W A Arrindell en C Schaap The Maudsley Marital Questionnaire (MMQ): an extension of its construct validity. The British Journal of Psychiatry: The Journal of Mental Science 147 (September 1985): 295299.

15. J Orathinkal, A Vansteenwegen, en R Stroobants,Further validation of the Maudsley Marital Questionnaire (MMQ). Psychology, Health \& Medicine 12, no. 3 (May 2007): 346-52.

16. K. Mah en Y M Binik, Are orgasms in the mind or the body? Psychosocial versus physiological correlates of orgasmic pleasure and satisfaction. Journal of Sex \& Marital Therapy 31, nr. 3 (June 2005): 187-200.

17. L R Derogatis, R S Lipman, en L Covi, SCL-90: an outpatient psychiatric rating scale--preliminary report. Psychopharmacology Bulletin 9, nr. 1 (January 1973): 13-28.

18. G Sintchak en J H Geer, A vaginal plethysmograph system. Psychophysiology 12, nr. 1 (January 1975): 113-115.

19. E Laan, W Everaerd, en A Evers, Assessment of female sexual arousal: response specificity and construct validity. Psychophysiology 32, no. 5 (September 1995): 476-485.

20. R J Levin en R P Macdonagh, Increased vaginal blood flow induced by implant electrical stimulation of sacral anterior roots in the conscious woman: a case study. Archives of Sexual Behavior 22, no. 5 (October 1993): 471-5.

21. J H Geer en Eric Janssen, The Sexual Response system. in Handbook of Psychophysiology, 2e ed. (Cambridge University Press, 2003), 315-340.

22. Rachel N Pauls e.a., Effects of sacral neuromodulation on female sexual function. International Urogynecology Journal and Pelvic Floor Dysfunction 18, nr. 4 (April 2007): 391-395.

23. Giuseppe Lombardi e.a., Clinical female sexual outcome after sacral neuromodulation implant for lower urinary tract symptom (LUTS). The Journal of Sexual Medicine 5, no. 6 (June 2008): 1411-7.

24. N Zabihi e.a., The effects of bilateral caudal epidural S2-4 neuromodulation on female sexual function. International Urogynecology Journal and Pelvic Floor Dysfunction 19, no. 5 (May 2008): 697700.

25. Ingber et al., Neuromodulation and female sexual function. International Urogynecology Journal and Pelvic Floor Dysfunction 20, no 9 (September 2009):1055-1059

26. Spinelli et al., A new minimally invasive procedure for pudendal nerve stimulation to treat neurogenic bladder. Neurourology and Urodynamics 24, no. 4 (2005);305-309

27. M E D Jarrett, R J Nicholls, en M A Kamm, Effect of sacral neuromodulation for faecal incontinence on sexual activity. Colorectal Disease: The Official Journal of the Association of Coloproctology of Great Britain and Ireland 7, no. 5 (September 2005): 523-525.

28. M L Chivers e.a., Agreement of self-reported and genital measures of sexual arousal in men and women: a meta-analysis. Archives of Sexual Behavior 39, nr. 1 (February 2010): 5-56.

29. A H Rellini e.a., The relationship between women's subjective and physiological sexual arousal. Pychophysiology 42, nr. 1 (January 2005): 116-124. 
30. S Meloy, Neurally augmented sexual function. Acta Neurochirurgica. Supplement 97, no. Pt 1 (2007): 359-363.

31. B Govaert et al., The effect of sacral nerve stimulation on uterine activity: a pilot study. Colorectal Disease: The Official Journal of the Association of Coloproctology of Great Britain and Ireland 12, no. 5 (May 2010): 448-451. 
Chapter 8

Discussion and future perspectives 
CHAPTER 8 
Lower urinary tract symptoms have a significant impact on the quality of life of affected persons. ${ }^{1}$ Before the introduction of SNM, therapeutic options for refractory OAB symptoms were limited to invasive and irreversible surgical procedures, such as ileocystoplasty or urinary deviation procedures with considerable complication rates. Patients with urinary retention are dependent on self-catheterization, which forms a daily inconvenience.

The results of his thesis show that SNM can give lasting improvement in patients with symptoms of $O A B$ and non-obstructive urinary retention (Chapter $\mathbf{2}$ and Chapter 3).

But there still is a considerable group of implanted patients that do not benefit optimally from SNM.

Optimizing selection of patients who have the greatest likelihood of having benefit from an SNM system remains a challenge.

A study has been performed on predictive factors for succes of the PNE test. Scheepens et al studied predictive factors of having a positive PNE and found the following positive predictors: having had a intravertebral disk repair, urinary complaints over 7 months before undergoing PNE, and having OAB wet. A negative predictor was having neurogenic bladder dysfunction. ${ }^{2}$

Several studies have been carried out into predictors of the results of definitive SNM implants.

The predictive value of the age at which SNM implant takes place remains an issue of debate, as there are contradictory study results.

Amundsen et al found that having an age older than 55 and comorbidity of more than three chronic diseases were associated with lower success rates of SNM in urgency symptoms. ${ }^{3}$ However, in another study in 19 elderly females with OAB patients in 2009, the success rates and complication rates that were found were as described in this thesis, but device removal rates were increased. ${ }^{4}$

In conclusion, there are no unambiguous data yet that allow for a preselection of candidates for SNM. Therefore the temporary test procedure is the only currently known method to predict outcome of the definitive SNM implant and to select appropiate candidates.

The usual method of determining whether a patient is a responder to SNM is the PNE test.

The average success rate of the PNE lies around $50 \% .^{5,6}$ In Chapter 4 our first experiences with temporary testing with the Tined Lead are described. This new lead has as advantages that it can be implanted in a day care surgery setting and test lead migration occurs less often due to fixation of the lead with silicone barbs in the fascia. This allows for longer testing, compared to the PNE. The maximal duration of the conventional PNE is up to a week and with the Tined lead an average testing period was 12 days, with an outlier of 31 days without complications. 
The overall success rate of testing through the Tined Lead of $70 \%$ to $80 \%$ in this thesis, is confirmed by other authors. ${ }^{7,8,9}$ Therefore testing through the Tined Lead seems to be superior over the conventional PNE when considering the increase of the chance of a positive test. Furthermore, the electrode configuration leading to a positive outcome during the test can be carried over after definitive implant, as the testing lead is used as a definitive lead. However, comparison of long term results of definite implants after a positive PNE versus a positive Tined Lead test does not show a significant difference in ultimate outcome. ${ }^{9}$

Despite the increase of likelihood of a positive result after Tined Lead testing compared to the PNE, the PNE still retains its value as a test method.

The costs of the conventional PNE are considerably lower than the costs of the Tined Lead prodecure. It can be carried out in an outpatient setting and is therefore more easily accessible. Testing with the Tined Lead requires an operating room and fluoroscopy. The PNE also allows for an easily accessible manner to determine whether patients can handle the stimulation equipment and are willing and able to follow the instructions and to maintain adequate voiding diaries.

In our single center study in Chapter 3, we have concluded that technical improvements led to decrease of complication rates. The stimulation equipment has improved, as the implantable pulse generator (IPG) became more advanced.

The Itrell-I model used a strong magnet to allow the patient to switch the stimulator off, while later models were supplied with a Patient Programmer that works through telemetry. In addition to switching the stimulator on and off, it also allows for changing stimulation amplitude. In the first version of the IPG, changing stimulation settings could only be done in a surgical procedure.

In 2006, the latest Interstim-II IPG, model 3058 became available. Its volume of 14 $\mathrm{cm} 3$ is considerably reduced, compared to its predecessor, the InterStim-II model 3023 with a volume of $22 \mathrm{~cm} 3$. Solid long term results of the new IPG are not available, but it is expected that the rate of pain complaints at the IPG site will decrease, especially in physically active patients.

During the development of SNM, implant techniques improved as well. Classically, the SNM system was implanted through an open surgical procedure, with direct placement of the lead and suturing it to the sacral periost. The IPG was placed in the left or right lower abdominal quadrant. The buttock placement, ie. placing the IPG about $5 \mathrm{~cm}$ caudally to the iliac crest, gave less pain at the IPG site at followup. ${ }^{10}$ Operation time decreased from 2.5 hour to 1-1.5 hour as patients required no more turns on the operating table during surgery.

Currently, the open procedure has been replaced in virtually each centre by minimal invasive implant with the Tined Lead. Placement occurs under local anesthesia, therefore both sensory and motor responses can be used to determine optimal lead placement. 
The literature seems to be in agreement that a motor response is required, at least during testing, to achieve efficacy of stimulation. ${ }^{8,11}$ But it is not clear what the significance of a sensory response is for achieving improvement of voiding symptoms. Both during the test phase and after definitive implant, the stimulation amplitude can be regulated by the patient to such a voltage that patients feel the stimulation, but not in an unpleasant manner. However in our clinical experience, there are patients that have benefit of SNM without feeling the stimulation. The exact role of the patient's sensory perception during test procedures and during stimulation by a definitive implant is still under debate.

In the follow-up visits after SNM implant, sensation plays a role in troubleshooting. In most reprogramming sessions the sensory response of the patient is the only guide for choosing new stimulation settings.

In the clinical practice is a distinction between patients with good results of SNM with or without feeling the stimulation. For patients with SNM for faecal complaints Koch et al. have found that patients can have positive results when stimulated below sensory threshold. ${ }^{12}$

Peters et al investigated the relation between a sensory response during lead implant and the outcome of SNM after 24 months. ${ }^{13}$ They found no significant difference in outcomes between patients with and patients without a sensory response during implant. About $32 \%$ of the patients included in the study were implanted for interstitial cystitis. Care must be taken to extrapolate this results to the SNM population as the proportion of interstitial cystitis patients in the study is large, compared to the general SNM population.

The research of the working mechanism of SNM seems to point to changes in cerebral structures that process afferent information from the bladder. ${ }^{14,15}$ It would be interesting to research the role of patient's sensation on the efficacy of chronic SNM in long term studies with sufficient study subjects.

Migration rates of the Tined lead, used as definitive lead, were found to be $2.1 \%$ in one long term study with 200 implanted patients and in another study with 64 patients the migration rate was $1.6 \% .^{16,17}$

This figure is lower than the proportion of suspected lead migration of $3.3 \%$ found in the long term study with the conventional lead in Chapter 2.

When placing the lead at the correct depth, the tines are placed in the subcutaneous tissue. It is assumed that fixation of the tines takes place due to fibrosis. In the short term, for example in the case of a failed first stage procedure, the lead can easily be removed by applying moderately firm traction perpendicular to the sacrum. In a situation after a definitive implant, forces as such will not occur. Furthermore, after both classical method and after minimal invasive implant, patients are advised to avoid bending and sudden movements of their backs for six weeks. In both studies describing lead migration, change of physical activity or 
change in body weight are not mentioned in patients with lead migration. As the tines of the Tined Lead fixate in the subcutaneous tissue, changes in properties of this tissue or the surrounding musculature might predispose to lead migration. When this hypothesis is confirmed in further studies, patients can be counselled regarding lifestyle changes and the chance of lead migration.

The initial stimulation parameters that are used are as follows: continuous stimulation with a frequency of $10 \mathrm{~Hz}$ and a pulse width of $210 \mu \mathrm{sec}$.

These parameters have been arbitrarily chosen and until now little research has been performed on efficacy of different parameter settings. Marcelissen et al have evaluated four different pulse rates in fifty patients with suboptimal effect. Eighteen out of 22 patients with stimulation related pain with stimulation at $10 \mathrm{~Hz}$ had pain relief when stimulated with another frequency. In several younger patients, stimulation at $40 \mathrm{~Hz}$ led to significant improvement in voiding parameters. ${ }^{18}$ The results by Marcelissen et al show that an algorithm for tailoring the pulse rate settings in individual patients may improve outcomes and decrease adverse events, such as stimulation related pain. Until now, there are no studies into the effect of changes in pulse width or frequency on outcomes of SNM.

Most patients are advised to leave their stimulation system on for 24 hours per day and to switch it of when driving or when working with heavy machinery. This advise is given to prevent accidents by sudden increase of stimulation sensation due to swaying of the end of the electrode towards the nerve.

In our clinical experience there are patients who keep their favourable results after switching their IPG of or after temporary testing.

In Chapter $\mathbf{5}$ we have investigated the feasibility of neuromodulation on demand. We found that 10 out of 16 patients were able to have their system switched off for at least on average 12.4 hour per day without worsening of symptoms. It is not clear why there were patients who were able to interrupt their stimulation without recurrence of symptoms and who were not able to do so.

A few patients felt comfortable with manually controlling their SNM system. We expected that patients would find controlling the stimulation bothersome. An explanation might be that patients whose daily lives were first centered around their urinary symptoms become symptom free, but depend on their stimulation system to achieve symptom resolution. Giving the patients more control over their SNM system might improve their feeling of self-efficacy. Research into long term efficacy in on demand SNM is desirable, but interesting as well would be to assess differences in quality of life between patients with continuous SNM versus on demand SNM. Furthermore, on demand SNM may increase battery life of the IPG and delay the necessity for surgical IPG replacement. 
One of the hypotheses in the on demand SNM study was that interruption of stimulation would prevent adaptation of the nerve system to the stimulation. In the literature little is known about the effects of prolonged continuous stimulation on peripheral nerves and on cerebral structures.

Once patients achieve good results with the test procedure and have a definitive implant, globally $70 \%$ keep their good results at a follow-up of up to five years. This means that $30 \%$ lose their initially favorable results. This group of patients form a challenge for the team involved in SNM.

First step of troubleshooting is ruling out technical failures as a cause of nonresponding.

Lead migration can be diagnosed with plain radiography. The IPG can measure the impedance in the stimulation circuit. A low impedance $(<400 \Omega)$ is indicative for a short circuit. This may happen in case of a damaged lead. Should the impedance be too high ( $>1500 \Omega$ ), then a lead breakage or a loose connection may be the case.

In most of the $30 \%$ of the patients with loss of efficacy, however, technical problems are ruled out.

McLennan measured compound muscle action potentials (CMAPs) at the anal sphincter in 10 female patients to determine the optimal course of action. ${ }^{19}$ The CMAPs were measured for each electrode configuration. The electrode configuration with the CMAP with the highest amplitude was deemed optimal. If a patient with loss of efficacy had an optimal configuration, then reprogramming was deemed useless. This was the case in 2 patients. If the configuration was not optimal, then the optimal settings were chosen in 5 patients. If no response was found, a lead migration was suspected.

All patients in this study had their loss of efficacy after a relatively short period after implant. The mean period before loss of SNM efficacy was 5.3 months. Therefore it would be interesting to determine whether this method of troubleshooting would also be valid for patients with loss of efficacy after a longer period of SNM.

Ghazwani et al found in their patient series of 143 patients 9 patients with loss of efficacy. ${ }^{20}$ These patients had required higher amplitudes ( $4 \pm 1.2 \mathrm{~V}$ vs. $2.83 \pm 0.7 \mathrm{~V}$ ) during the PNE procedure. The amplitude at implant and the current amplitude ware increased as well.

Impedances at the time of loss of efficacy were also higher in the non-responders: $(1032.4 \pm 181 \Omega$ vs. $590 \pm 44.6 \Omega$ ). The most obvious explanation may be that the amplitude requirement and impedance is increased due to fibrosis between the lead and the nerve, but that does not explain the higher amplitudes during the acute PNE. It would be interesting to see whether this association is also seen in other SNM centers.

These two studies investigated the peripheral nerves, but there is no data on cerebral mechanisms in non responders available. It is clear that in patients with SNM 
cerebral mechanisms are also involved. ${ }^{14,21,22}$ A PET-CT or fMRI study comparing cerebral perfusion areas in patients with loss of efficacy versus effect maintainers might be of interest.

In clinical experience with patients with lower urinary tract symptoms, a proportion of patients have psychological complaints or a psychiatric history. In the literature there are indications for a relation between a psychiatric history and an increased chance of loss of efficacy. Weil et al found that in patients with a psychiatric history, the effect of SNM lasted with a median of 12 months and in the remaining patients without a psychiatric history the effect lasted over 36 months. ${ }^{23}$ Furthermore the number of re-operations was higher in patients with a psychiatric history.

Marcelissen et al prospectively investigated the correlation between questionnaires into psychological factors and the results of the PNE and definitive implant. ${ }^{24}$ No relation between the score on the questionnaires and the outcome has been found. But there was a significant relation between a psychiatric history and the number of adverse events. Patients with a psychiatric history had $75 \%$ chance of an adverse event, while non-psychiatric patients had $13 \%$ chance of an adverse event. However, the duration of the follow-up was not mentioned in the article.

In 2009 White et al could not find a significant correlation between a psychiatric history and increased rate of adverse events in 221 patients with SNM, but did find an increased rate of SNM implant removal in patients with a psychiatric history. ${ }^{25}$ In conclusion, there seems to be a relation between having a psychiatric history and the occurrence of adverse events and SNM explant rates. It would be useful to further investigate the cause of the increase in adverse events in this group of patients. This may lead to better pre-implant patient counselling or the discovery of risk factors that may be modifiable.

Chapter 6 describes the study into sexual function of female patients with SNM. In our clinic, several female patients mentioned that their sexual functioning improved after SNM. The study was devised to verify the hypothesis that SNM does improve sexual function in patients with lower urinary tract or faecal symptoms.

The second objective was to determine whether the improvement was due to a physiological reaction on SNM of because of an improved well-being. Due to a low number of study subjects, no significant results have been found. Although the phletysmography results and the trend towards improvement of the Mah Orgasm Score may indicate a physiological reaction on SNM.

Reasons for the non-significant results are the low number of included patients and by the fact that our included patients probably experienced minimal problems with sexual functioning. This is a consequence of the inclusion criteria in the study; patients had to be sexually active to be able to fill in the questionnaires correctly. 
Pauls et al did find improvement in sexual function after SNM implant, but could not differentiate between the mechanism of improvement either. ${ }^{26}$

In their study an inclusion criteria was that patients had to be sexually active as well.

To have a better understanding, it would be useful to perform a study into improvement with SNM in patients who have known sexual dysfunction.

\section{FUTURE PERSPECTIVES}

From its introduction about twenty years ago up to now, SNM has seen a quick acceptance and remarkable growth. It has found its place between first pharmacological treatment and invasive surgical procedures for patients with refractory, invalidating symptom complexes.

The challenge for the future lies in optimizing SNM therapy to decrease the rate of patients who lose efficacy of their implant. One method of achieving this is optimization of patient selection.

While testing with the Tined Lead increases the chance of a successful test procedure, it does not seem to have an impact on post implant loss of efficacy. A partial solution may lie in the selection of patients who undergo the temporary test. Further research has to be carried out regarding additional pretest screening instruments.

It would also be interesting to determine whether psychological counselling before or during definitive SNM implant would be useful. This counselling may consist of the management of patient's expectations towards SNM results.

Several studies into optimization stimulation parameters, for example the on demand study in this thesis and the study into rate changes by Marcelissen et al., show that subgroups of the research population might benefit from other SNM parameters than the usual parameters. ${ }^{20}$

A proposed hypothesis for this phenomenon is that the causes of LUTS are multifactorial and factors might have a different expression in individual patients. In addition, causative factors can react differently on SNM. This may account for the differences in response in SNM and for differences in reaction on changes in stimulation parameters.

Therefore, besides more research into the causes on LUTS and working mechanism of SNM, research into an algorithm for customizing parameters as pulse rate, pulse width or continuous stimulation versus on demand stimulation in individual patients may yield better long term outcomes.

The indications of SNM are expanding. In addition to idiopathic OAB and nonobstructive urinary retention SNM is also successfully used in faecal incontinence or 
constipation, interstitial cystitis and pelvic pain syndromes and in patients with neurogenic voiding complaints. ${ }^{27,28,29}$

SNM is successfully used in patients with incomplete spinal injury. ${ }^{30}$ Recently Sievert et al published a study on bilateral SNM in 10 patients with complete spinal cord injury during the spinal shock phase. ${ }^{31}$ After a mean follow-up of 26 months, there was a decrease of urinary incontinence and urinary tract infections, compared to 6 control patients. Average catheterized volume in the intervention group was $582 \mathrm{ml}$ per catheterization $(480-650 \mathrm{ml}$ ) versus $208 \mathrm{ml}$; $(57-314 \mathrm{ml})$ in the control group. Patients were also not dependent on laxatives for defacation.

Usually, defaecation needs to be pharmacologically regulated and unpredictable bowel movement is a very troublesome consequence after spinal cord injury. Two male patients were able to achieve penile erection with specialized SNM settings.

These results seem promising and may have considerable impact on bladder and bowel management in spinal cord injury.

Another study in patients with spinal cord injury by Horvath et al in 2011 researched the feasibility of conditional pudendal neuromodulation. ${ }^{32}$ In 9 patients at least a year after initial complete or incomplete spinal cord injury,, a closed-loop system was used to record detrusor pressure during artificial bladder filling and to apply electrical stimulation of the pudendal nerve when the detrusor pressure rose above $40 \mathrm{cmH} 2 \mathrm{O}$. In 8 patients the average cystometric capacity increased, compared to no stimulation and in 6 patients there was a non-significant increase compared to continuous stimulation.

This study shows that conditional neuromodulation is feasible, at least in a laboratory situation. Further refinement and a different method of acquiring detrusor pressure changes might make a closed-loop system for conditional neuromodulation a feasible and useful therapy option in patients with lower urinary tract symptoms.

More research in responding and non-responding in patients with SNM for neurogenic LUTS might give more insight in the working mechanism of SNM. 


\section{REFERENCES}

1. A. K. Das, A. M. Carlson, and M. Hull, Improvement in Depression and Health-related Quality of Life After Sacral Nerve Stimulation Therapy for Treatment of Voiding Dysfunction. Urology 64, no. 1 (2004): 62-8.

2. W A Scheepens et al., Predictive Factors for Sacral Neuromodulation in Chronic Lower Urinary Tract Dysfunction. Urology 60, no. 4 (October 2002) 598-602.

3. Cindy $L$ Amundsen et al., Sacral Neuromodulation for Intractable Urge Incontinence:Are There Factors Associated with Cure? Urology 66, no. 4 (October 2005): 746-750.

4. Wesley $M$ White et al., Sacral Nerve Stimulation for Refractory Overactive Bladder in the Elderly Population. The Journal of Urology 182, no. 4 (October 2009): 1449-1452.

5. H. S. Shaker and M. Hassouna, Sacral Nerve Root Neuromodulation: An Effective Treatment for Refractory Urge Incontinence. J Urol 159, no. 5 (1998): 1516-1519.

6. R. A. Janknegt et al., Long-term Effectiveness of Sacral Nerve Stimulation for Refractory Urge Incontinence. Eur Urol 39, no. 1 (2001): 101-106.

7. Andreas Bannowsky et al., Urodynamic Changes and Response Rates in Patients Treated with Permanent Electrodes Compared to Conventional Wire Electrodes in the Peripheral Nerve Evaluation Test. World Journal of Urology 26, no. 6 (December 2008): 623-626.

8. Brian L. Cohen, Hari S.G.R. Tunuguntla, and Angelo Gousse, Predictors of Success for First Stage Neuromodulation: Motor Versus Sensory Response. The Journal of Urology 175, no. 6 (June 2006): 2178-2181.

9. Tom Marcelissen et al., Is the Screening Method of Sacral Neuromodulation a Prognostic Factor for Long-term Success? The Journal of Urology 185, no. 2 (February 2011): 583-587.

10. W. A. Scheepens et al., Buttock Placement of the Implantable Pulse Generator: a New Implantation Technique for Sacral Neuromodulation--a Multicenter Study. Eur Urol 40, no. 4 (2001): 434-438.

11. C. J. Fowler et al., Studies of the Latency of Pelvic Floor Contraction During Peripheral Nerve Evaluation Show That the Muscle Response Is Reflexly Mediated. J Urol 163, no. 3 (2000): 881-883.

12. S M P Koch, W G van Gemert, and C G M I Baeten, Determination of Therapeutic Threshold in Sacral Nerve Modulation for Faecal Incontinence, The British Journal of Surgery 92, no. 1 (January 2005): 83-87.

13. Kenneth M Peters, Kim A Killinger, and Judith A Boura, Is Sensory Testing During Lead Placement Crucial for Achieving Positive Outcomes After Sacral Neuromodulation? Neurourology and Urodynamics 30, no. 8 (November 2011): 1489-1492.

14. Bertil F M Blok et al., Different Brain Effects During Chronic and Acute Sacral Neuromodulation in Urge Incontinent Patients with Implanted Neurostimulators. BJU International 98, no. 6 (December 2006): 1238-1243.

15. Ranan Dasgupta et al., Changes in Brain Activity Following Sacral Neuromodulation for Urinary Retention. The Journal of Urology 174, no. 6 (December 2005): 2268- 2272.

16. Donna Y. Deng et al., Failure of Sacral Nerve Stimulation Due to Migration of Tined Lead. The Journal of Urology 175, no. 6 (June 2006): 2182-2185.

17. Tom A T Marcelissen et al., Long-term Results of Sacral Neuromodulation with the Tined Lead Procedure. The Journal of Urology 184, no. 5 (November 2010): 1997-2000.

18. Tom A T Marcelissen et al., The Effect of Pulse Rate Changes on the Clinical Outcome of Sacral Neuromodulation. The Journal of Urology 185, no. 5 (May 2011): 1781-1785.

19. M. T. McLennan, The Role of Electrodiagnostic Techniques in the Reprogramming of Patients with a Delayed Suboptimal Response to Sacral Nerve Stimulation. Int Urogynecol J Pelvic Floor Dysfunct 14, no. 2 (2003): 98-103.

20. Yahya Q Ghazwani, Mohamed S Elkelini, and Magdy Hassouna, Association Between Stimulation Parameters and Loss of Efficacy of Selective Sacral Nerve Root Stimulation. Neuromodulation: Journal of the International Neuromodulation Society 14, no. 3 (June 2011): 266-270. 
21. Rajesh Kavia et al., A Functional Magnetic Resonance Imaging Study of the Effect of Sacral Neuromodulation on Brain Responses in Women with Fowler's Syndrome. BJU International 105, no. 3 (February 2010): 366-372.

22. P. M. Braun et al., Alterations of Cortical Electrical Activity in Patients with Sacral Neuromodulator, Eur Urol 41, no. 5 (2002): 562-6.

23. E. H. Weil et al., Clinical Results of Sacral Neuromodulation for Chronic Voiding Dysfunction Using Unilateral Sacral Foramen Electrodes. World J Urol 16, no. 5 (1998): 313-21.

24. Tom A T Marcelissen et al., Psychological and Psychiatric Factors as Predictors for Success in Sacral Neuromodulation Treatment. BJU International 108, no. 11 (December 2011): 1834-1838.

25. Wesley $M$ White et al., Incidence and Predictors of Complications with Sacral Neuromodulation. Urology 73, no. 4 (April 2009): 731-735.

26. Rachel N Pauls et al., Effects of Sacral Neuromodulation on Female Sexual Function.International Urogynecology Journal and Pelvic Floor Dysfunction 18, no. 4 (April 2007): 391-395.

27. M E D Jarrett et al., Systematic Review of Sacral Nerve Stimulation for Faecal Incontinence and Constipation. The British Journal of Surgery 91, no. 12 (December 2004): 1559-1569.

28. Craig V Comiter, Sacral Neuromodulation for the Symptomatic Treatment of Refractory Interstitial Cystitis: a Prospective Study. The Journal of Urology 169, no. 4 (April 2003): 1369-1373.

29. Markus Hohenfellner et al., Chronic Sacral Neuromodulation for Treatment of Neurogenic Bladder Dysfunction: Long-term Results with Unilateral Implants. Urology 58, no. 6 (December 2001): 887892.

30. G Lombardi and G Del Popolo, Clinical Outcome of Sacral Neuromodulation in Incomplete Spinal Cord Injured Patients Suffering from Neurogenic Lower Urinary Tract Symptoms. Spinal Cord 47, no. 6 (June 2009): 486-491.

31. Karl-Dietrich Sievert et al., Early Sacral Neuromodulation Prevents Urinary Incontinence After Complete Spinal Cord Injury. Annals of Neurology 67, no. 1 (January 2010): 74-84.

32. Eric E Horvath et al., Conditional and Continuous Electrical Stimulation Increase Cystometric Capacity in Persons with Spinal Cord Injury. Neurourology and Urodynamics 29, no. 3 (March 2010): 401-407. 
Chapter 9

Summary 
CHAPTER 9 
Chapter 1 starts with a brief description of the burden that LUTS give on the patients and on society. Symptoms of dysfunctional voiding are rather common and have a significant impact on quality of life in patients and have considerable economical consequences in society. Chapter 1 also contains an overview on the history and the procedure of Sacral neuromodulation (SNM). SNM is a treatment of voiding symptoms by stimulating the nerve root that runs through the third foramen of the sacral bone. The exact working mechanism is still not known, but chapter 1 provides an overview of the existing knowledge and current research on this subject.

Furthermore, the aims of this thesis are outlined.

Chapter 2 gives an overview on the neurophysiology of normal voiding. Storage and disposal of urine are controlled by several reflex arcs in cerebro and in the spinal cord, controlling a finely tuned coordination between bladder and sphincter complex. The pathophysiology of overactive bladder symptoms and of nonobstructive urinary retention that can be eligible for treatment by SNM is discussed. In addition, a few more recent indications for SNM are described.

Chapter 3 presents the results of a prospective multicenter study on the effect of SNM with a follow-up period of 5 years. Clinical success of SNM is arbitrarily defined as at least $50 \%$ improvement in relevant voiding parameters, measured with voiding diaries. Clinical success is used as a criterion for a succesful test procedure, while in this study it is used as an end point to describe stimulation efficacy. The clinical success rate after 5 year follow-up is $68 \%$ for patients with urgency incontinence, $56 \%$ in patients with urgency-frequency and $71 \%$ in patients with retention. Furthermore, the rate of adverse events and reoperation rate are reported. There were no life threatening or irreversible events and $67 \%$ of the implanted patients had at least one adverse event. $39,5 \%$ of the patients needed surgical correction of an adverse event, including the need for stimulator replacement because of battery depletion.

Chapter 4 describes the results of a retrospective study on long term outcome at the Department of Urology in the Academisch Ziekenhuis in Maastricht.

This study included 149 patients, 107 had overactive bladder symptoms and 42 had urinary retention. Mean follow-up was 64.2 ( $\mathrm{sd}=38.5$ ) months. In the whole group 194 adverse events occurred. 6 Patients had an infection in their implanted system, but no patient was explanted for infection. Most events could be solved by giving advice or by reprogramming the stimulator. 129 Re-operations have been performed and 21 patients had their system explanted.

A difference in in the incidence of re-operations was found between the groups of patients implanted before or after 1996. However, this did not reflect on the 
subjective results, these stayed equal between those groups. Reasons given for the difference in re-operation rates were: a learning curve in initial SNM therapy and technical and procedural improvements.

Chapter 5 shows initial results and median term follow-up with a new lead that can be implanted in a minimally invasive procedure under local anesthesia. This allows for temporary testing where, in the case of a positive result, the lead can function as a definitive lead. 49 patients, 39 with refractory overactive bladder symptoms, 10 with urinary retention, were implanted with the tined lead under local anesthesia. The average test period was 12.4 days, (sd 5.8 days). Patients were implanted with an implantable pulse generator when they had at least $50 \%$ improvement in voiding diaries. Average follow-up for implanted patients was 15.5 months ( $s d=7.9$ months). Results: 10 patients had a single stage implant, 39 had a two-stage implant. Of 39 patients with 2-stage implant 31 (79.5\%) were positive, 8 (20.5\%) were not. 31 patients were included in follow-up. At last follow-up 28 (90.3\%) patients had more than $50 \%$ improvement in diary parameters and $3(9.7 \%)$ had not. Seven patients had an adverse event. One incomplete electrode migration was seen and was treated conservatively. The Tined Lead seems to give a higher chance of a positive test result than the conventional PNE and gives good median term results.

Chapter 6 investigates the feasibility of intermittent SNM. The general opinion is that patients need to be stimulated continuously to achieve their results.

However, there is anecdotal evidence that stimulation can be interrupted without losing efficacy on voiding symptoms. Intermittent stimulation could have as advantage that it might save battery life, and perhaps postpone loss of efficacy in the long term.

21 Patients were randomized in two groups; 16 patients in the on demand group and 5 patients were controls, in addition to a run in period of two weeks where each patient performed his or her own control. In the on demand stimulation 10 out of 16 patients seemed to be able to switch the stimulator off for on average 12.4 hours without increase of voiding symptoms. An unexpected beneficial side effect was that several patients felt more comfortable with on demand stimulation as it gives them a feeling of control over their voiding symptoms.

Chapter 7 shows the results of a study on the influence of SNM on sexual function in female patients, treated for urinary symptoms or faecal incontinence.

Eight patients were analyzed with an array of sexological questionnaires into sexual function and dysfunction and three of these eight patients underwent measurement of the vaginal pulse amplitude with vaginal phletysmography. 
Although no statistically significant results were found, the combination of the phletysmography results and a trend towards improvement of orgasm perception after SNM implant suggest a physiologically mediated improvement.

Inclusion of subjects was very slow, this may be because for inclusion current sexual activity was a criterion, in order to fill out the questionnaires, while sexual function may be deteriorated because of the voiding symptoms or faecal symptoms.

Chapter 9 concludes this thesis with a discussion on the implications of the found results. SNM has proven to be a save and effective therapy, but its results depend on careful selection of potential candidates. Until now, the only means of selecting those who will respond favourably on definitive SNM remains a temporary trail procedure.

Testing with the new minimal invasive electrode increases the rate of positive temporary test results, but it is not clear whether it will give better SNM results in the long term. Other means of optimizing long term results might be on demand stimulation and tailoring stimulation parameters towards the individual patient.

The indications for SNM are expanding and SNM might have a place in patients with neurogenic LUTS. Further research might be directed into further refinement of SNM technology and into additional selection criteria. 

Chapter 10

Nederlandse samenvatting 
CHAPTER 10 
Dit proefschrift gaat over de behandeling van chronische, veelal onbegrepen, klachten van het plassen met sacrale neuromodulatie. Sacrale neuromodulatie is een behandeling waarbij men de zenuw die door het derde foramen van het heiligbeen (het sacrum) loopt, stimuleert met elektrische pulsen. Deze techniek is in de 80-er jaren van het vorige decennium ontwikkeld door een groep onderzoekers in San Fransico.

Een neuromodulatiesysteem bestaat uit een in het heiligbeen geplaatste electrode. De elektrode is verbonden aan een onderhuids in de bil geïmplanteerde stimulator. De exacte werking van sacrale neuromodulatie is vooralsnog niet bekend. Wel is bekend dat de werking niet wordt veroorzaakt door directe stimulatie van blaas of urinewegen, maar dat de stimulatie veranderingen teweeg brengt in de voor het plassen verantwoordelijke reflexbogen in het ruggenmerg. Verder zijn er aanwijzingen dat neuromodulatie ook veranderingen geeft in activiteit van de hersenstructuren waarin het plassen geregeld wordt.

De plasklachten kunnen bestaan uit urgencyincontinentie, ook wel aandrangsincontinentie genoemd, urgency-frequency (het vaak en met grote aandrang kleine beetjes plassen) en het vasthouden van urine (retentie) zonder obstructie beneden de blaas.

De patiënten met chronische blaasklachten die in aanmerking komen voor sacrale neuromodulatie vormen een lastig te behandelen groep. Het hebben van deze langdurige klachten is sociaal invaliderend doordat patiënten afhankelijk zijn van incontinentiemateriaal of hun activiteiten moeten plannen op basis van het in de buurt zijn van een toilet. Patiënten met retentie zijn afhankelijk van zelfcatheterisatie, wat een aanzienlijk dagelijks ongemak geeft. In beide gevallen zijn ook de kosten die de aandoening met zich meebrengt, aanzienlijk.

In het geval van aandrangsklachten wordt er gestart met adviezen ten aanzien van vochtinname, eventueel aangevuld met bekkenbodemfysiotherapie. Bij onvoldoende effect worden blaasremmende medicamenten gegeven. Het nadeel hiervan is dat deze systemisch werken en daardoor in veel patiënten hinderlijke bijwerkingen geven, waardoor het percentage patiënten dat na enige tijd met de medicatie stopt hoog is.

Voor de introductie van de sacrale neuromodulatie was de laatste optie operatieve behandelingmet blaascapaciteitvergrotende technieken of blaasvervangingen. Deze operaties zijn onomkeerbaar en kennen een aanzienlijk complicatiepercentage.

Sinds de introductie van de neuromodulatie in de jaren 90, heeft deze therapie zijn plaats gevonden in de rij mogelijkheden voor de patiënten met plasklachten. De techniek kent goede resultaten en is veilig en omkeerbaar.

De procedure voor neuromodulatie is als volgt.

Als een patiënt kandidaat voor neuromodulatie lijkt te zijn, vindt eerst een testprocedure, de Perpiheral Nerve Evaluation (PNE), plaats. Een tijdelijke elektrode, verbonden aan een uitwendige stimulator, wordt geplaatst. Na ongeveer een week 
worden door de patiënt bijgehouden plasdagboekjes van voor de stimulatie vergeleken met tijdens de stimulatie. Bij $50 \%$ of meer verbetering van de parameters in de plasdagboekjes wordt in overleg met patiënt besloten voor implantatie van een definitief systeem.

In de beginperiode werd deze implantatie gedaan in een operatie onder algehele anesthesie. Implantatie van de elektrode gebeurde met een incisie over het sacrum, waarna de elektrode geplaatst werd en verankerd aan het bot.

Sinds 2004 wordt gebruik gemaakt van een minimaal invasieve elektrode plaatsing die kan plaatsvinden onder lokale verdoving. Hierdoor kan de elektrode die definitief geplaatst kan worden ook gebruikt worden als testelektrode.

Het doel van dit proefschrift is het in kaart brengen van de lange termijnresultaten en de complicaties van sacrale neuromodulatie. Verder worden de eerste testresultaten op de afdeling Urologie van het Academisch Ziekenhuis in Maastricht met de minimaal invasieve elektrode beschreven. Er wordt een onderzoek beschreven naar toepasbaarheid van onderbroken stimulatie in plaats van de gebruikelijke continue stimulatie. Voordelen hiervan kunnen zijn dat de batterij van de stimulator langer meegaat, zodat het operatief wisselen van de stimulator uitgesteld kan worden. Verder blijkt dat op langere termijn een aanzienlijk percentage patienten geen effect van de stimulatie meer heeft, terwijl er wel adequaat gestimuleerd wordt. Mogelijk komt dit door aanpassingen in het zenuwstelsel aan de langdurige stimulatie. Als dit het geval is, zou onderbroken stimulatie ervoor kunnen zorgen dat dit effect uitgesteld wordt.

Bij de controlebezoeken aan de poli door patiëntes met een geïmplanteerd neuromodulatie systeem werd door enkele vrouwelijke patiënten spontaan verbetering van het sexuele functioneren gemeld. In dit proefschrift is een studie gedaan met als doel om na te gaan of er inderdaad verbetering van sexueel functioneren is bij vrouwelijke patienten met neuromodulatie en of deze eventuele verbetering veroorzaakt wordt door effecten van stimulatie van de zenuw die onder andere naar de geslachtsorganen loopt of door een verbeterd welbevinden doordat de plassymptomen zijn opgelost.

Hoofdstuk 1 bevat een introductie voor dit proefschrift. Het vóórkomen en de gevolgen van blaasklachten wordt beschreven. Verder wordt een overzicht gegeven van het tot nu bekende onderzoek naar de werking van neuromodulatie. Hoofdstuk 1 bevat ook een korte beschrijving van de historie en de procedure van sacrale neuromodulatie.

Hoofdstuk 2 beschrijft de neurofysiologie van het normale plassen. De controle van opslag en evacuatie van urine wordt geregeld door een goed gecoördineerd netwerk van reflexen in ruggenmerg en brein. Ook wordt de pathofysiologie van de 
belangrijkste indicaties voor sacrale neuromodulatie, te weten symptomen van een overactieve blaas en urineretentie, besproken. Ook worden enkele recentere indicaties besproken.

Hoofdstuk 2 beschrijft de resultaten van een grootschalig prospectief multicenter onderzoek naar de lange termijnresultaten van sacrale neuromodulatie. 152 Patienten met urgency incontinentie, urgency/frequency of urineretentie die waren geïmplanteerd met een neuromodulatiesysteem werden voor vijf jaar jaarlijks gevolgd met behulp van analyse van plasdagboekjes. Verder werden de complicaties en heroperaties bijgehouden. De stimulatie werd als succesvol gezien wanneer er $50 \%$ verbetering was in dagboekparameters bij het laatste bezoek, vergeleken met de parameters voor implantatie. Succesvolle stimulatie na 5 jaar werd bij $68 \%$ van de patiënten met urgency incontinentie, $56 \%$ van de patiënten with urgency-frequency en in $71 \%$ van de patiënten met retentie vastgesteld. $\mathrm{Er}$ hadden zich tijdens de 5 jaar follow-up geen levensbedreigende of onomkeerbare complicaties voorgedaan. $76 \%$ van de patienten had minstens één complicatie; 39,5 \% van de patiënten moesten geheropereerd worden in verband met een complicatie. In deze $39,5 \%$ zitten ook patiënten bij wie de stimulator moest worden vervangen omdat de batterij leeg was.

Er werd een sterke relatie gezien tussen het resultaat na 1 jaar en na 5 jaar, bij een positief resultaat na 1 jaar is de kans groot dat het resultaat na 5 jaar ook positief is.

Hoofdstuk 3 bevat een overzicht van de lange termijnresultaten van sacrale neuromodulatie op de afdeling Urologie in het Academisch Ziekenhuis in Maastricht.

Deze resultaten werden verkregen door statusonderzoek. De statussen van 149 patiënten werden nagezocht op resultaten en complicaties. Overactieve blaassymptomen waren de indicatie voor neuromodulatie in 107 patiënten; retentie in 42 patiënten. De gemiddelde follow-up bedroeg 64,2 (sd = 38,5) maanden. In de gehele groep werden 194 complicaties geregistreerd. Bij 6 patiënten werd een infectie rond het systeem gevonden, maar dit was bij geen van de patiënten reden tot verwijdering van het systeem. De meeste complicaties konden worden opgelost door adviezen te geven of het systeem te herprogrammeren. 129 heroperaties werden uitgevoerd en in 21 patiënten werd het systeem uiteindelijk verwijderd. Er was een opmerkelijk verschil tussen de aantallen heroperaties in patiënten die geïmplanteerd werden voor 1996 en in 1996 of later. Dit verschil werd niet teruggezien in de effectiviteit van neuromodulatie bij patiënten die voor of na 1996 werden geïmplanteerd. Hieruit werd geconcludeerd dat het aantal complicaties daalde door een leercurve en door verbeterde elektroden, stimulatoren en operatietechnieken. 
Hoofdstuk 4 is een onderzoek naar de eerste ervaringen van de afdeling Urologie in het Academisch Ziekenhuis in Maastricht met een nieuwe elektrode die in een minimaal invasieve procedure geplaatst kan worden onder lokale verdoving.

Hierdoor is de elektrode bruikbaar als tijdelijke testelektrode en bij goede testresultaten kan aan dezelfde elektrode een implanteerbare pulsgenerator gekoppeld worden. Doordat de kans op verschuiven van de elektrode kleiner is dan bij de PNE, kan er langer getest worden dan bij de standaard tijdelijke testelektrode. Er werden 49 patiënten met deze elektrode geïmplanteerd; 39 patiënten met overactieve blaassymptomen en 10 patiënten met retentie. De gemiddelde testperiode bedroeg 12,4 dagen ( $s d=5,8$ dagen) Patiënten werden met een neurostimulator geïmplanteerd bij $50 \%$ of meer verbetering in plasdagboekjes en gevolgd voor gemiddeld 15,5 maanden ( $s d=7,9$ maanden).

Bij 10 patiënten met een positieve standaard test werd de elektrode als definitieve elektrode geïmplanteerd. Bij de 39 andere patiënten hadden 31 patiënten (79,5\%) een positieve test en $8(20,5 \%)$ niet. Het percentage patiënten met een positieve test is hoger dan de $50 \%$ die bij de PNE wordt gezien. Verder hield 90,3\% van de 39 op de middellange termijn gevolgde patiënten hun positieve resultaat.

Bij 7 patiënten werd een complicatie gezien en bij 1 patiënt werd een verschuiving van de elektrode gezien, maar deze patiënt kon worden behandeld door herprogrammeren van de stimulator. De Tined Lead geeft een hogere kans op een positieve test voor sacrale neuromodulatie en de resultaten op middellange termijn zijn goed.

Hoofdstuk 5 laat de haalbaarheid van onderbroken neuromodulatie zien. De algehele opinie was dat patiënten 24 uur per dag continu gestimuleerd moeten worden om hun resultaten te houden. Wel wordt geadviseerd om de stimulator uit te zetten bij auto rijden of het bedienen van zware machines. Hiermee hoopt men ongelukken te voorkomen doordat de stimulatie ineens heftiger wordt bij onverwachte bewegingen. Dit leidt in de praktijk niet tot toename van plasklachten. Voordelen van onderbroken stimulatie kunnen zijn dat er zuiniger wordt omgegaan met de batterij van de stimulator, waardoor het operatief wisselen van de stimulator kan worden uitgesteld. Ook is er een aanzienlijk aantal patiënten waarbij na langere tijdsduur van sacrale neuromodulatie de plasklachten terugkomen, ondanks technisch adequate stimulatie. Een theorie die dit zou kunnen verklaren is dat het zenuwstelsel zich aanpast aan de stimulatie. Onderbroken stimulatie zou dit proces kunnen vertragen of misschien kunnen stoppen.

Zestien patiënten met twee weken continue en twee weken onderbroken stimulatie werden vergeleken met 5 controle patiënten en met zichzelf. De patiënten met onderbroken stimulatie werd gevraagd om de simulator uit te zetten en in te schakelen als zij weer klachten kregen. Verder hielden zij bij hoe lang de 
stimulator in- of uitgeschakeld was en werden in beide groepen plasdagboekjes bijgehouden.

In de onderbroken stimulatiegroep konden 10 van de 16 patiënten hun stimulator gemiddeld 12,4 uur per dag uit houden zonder noemenswaardige toename van klachten en verslechtering van de waarden in de plasdagboekjes.

Een effect dat wij niet hadden verwacht was dat enkele patiënten het prettig vonden om zelf controle te houden over wanneer het systeem aan of uit staat.

Hoofdstuk 6 beschrijft een onderzoek naar de invloed van sacrale neuromodulatie op het sexueel functioneren van vrouwelijke patiënten met klachten van een overactieve blaas, urineretentie of faecale incontinentie.

Voor en na de implantatie van het neuromodulatiesysteem werd een batterij van seksuologische vragenlijsten ingevuld door 8 patiëntes. Bij 3 van deze patiëntes werd voor en na de implantatie de vaginale doorbloeding gemeten in seksueel opgewonden en in neutrale toestand. Er werden geen statistisch significante resultaten gevonden, maar de combinatie van toegenomen orgasmescores en vaginale doorbloeding na implantatie lijken te wijzen op een verbetering die wordt veroorzaakt door een effect van de neuromodulatie op de vrouwelijke geslachtsorganen.

De studie kende een lange looptijd door moeizame rekrutering van proefpersonen. Oorzaak hiervoor is vermoedelijk het feit dat proefpersonen seksueel actief moeten zijn om de vragenlijsten goed te kunnen invullen, terwijl de blaasklachten of faecale klachten ertoe zouden kunnen leiden dat patiëntes niet seksueel actief meer zijn.

Hoofdstuk 9 sluit dit proefschrift af met een discussie over de implicaties van de gevonden resultaten. Sacrale neuromodulatie is effectief op de lange termijn bij de meeste patienten die behandeld worden met neuromodulatie voor urgency incontinentie, urgency-frequenct of urineretentie. De therapie vormt een waardevolle aanvulling bij de behandeling van deze hardnekkige en invaliderend klachten. Sacrale neuromodulatie is omkeerbaar en is veilig. Er treden weliswaar complicaties op, maar de meeste kunnen behandeld worden met advies of het herprogrammeren van het systeem. Tevens hebben technische ontwikkelingen van de elektrode, de implanteerbare pulsgenerator en verbeterde operatietechnieken zoals het implanteren van de pulsgenerator in de bil in plaats van in de onderbuik tot afname van complicaties geleid. Er blijft echter een aanzienlijke groep patiënten waarbij het effect van de neuromodulatie op langere termijn verloren gaat.

De enige manier om te bepalen of een patiënt in aanmerking komt voor neuromodulatie is vooralsnog een test met een tijdelijke elektrode. Probleem hierbij is de kans op verschuiving van de elektrode. Bij testen met de Tined Lead elektrode is de kans op verschuiving lager en kan langer getest worden. De kans op een positieve tijdelijke test is groter bij testen met de Tined Lead elektrode. Verder kan deze elektrode als definitieve elektrode gebruikt worden en patienten die met deze 
elektrode geïmplanteerd zijn, houden op de middellange termijn gelijkwaardige resultaten als met de conventionele elektrode. Aangezien testen met de conventionele elektrode logistiek gemakkelijker en laagdrempeliger is, is deze elektrode vooralsnog de eerste keus. Onderbroken neuromodulatie is een haalbare vorm van neuromodulatie, waarbij in een meerderheid van de patiënten geen toename van klachten gezien wordt. Verder vindt een groep patiënten het aangenaam om het gevoel van controle over de stimulatie te hebben. Mogelijk kan deze neuromodulatiemethode het eerder genoemde verlies van werkzaamheid van neuromodulatie tegengaan, maar meer onderzoek naar onderbroken neuromodulatie is nodig. Het zou interessant zijn om naar een algoritme voor de instellingen van sacrale neuromodulatie over te gaan, waarin in plaats van de standaardinstellingen, gekeken wordt naar instellingen op maat per patient.

Mogelijk zou dit tot nog minder uitval op lagere termijn en ongunstige bij/effecten kunnen leiden.

In de studie naar verbetering van seksueel functioneren van vrouwelijke patiënten die neuromodulatie hebben voor blaasklachten of faecale klachten is geen overtuigend bewijs gevonden dat neuromodulatie daadwerkelijk verbetering van seksueel functioneren geeft. Een reden hiervoor is het lage aantal proefpersonen. Wel lijkt er een direct effect te zijn op de vrouwelijke geslachtsorganen, maar verder onderzoek met meer proefpersonen is wenselijk.

Recent wordt er meer onderzoek gedaan naar de toepassing van sacrale neuromodulatie bij patienten met neurogene blaasklachten. Verder onderzoek naar de resultaten bij deze groep zou ook meer informatie kunnen geven onder het werkingsmechanisme. 
Chapter 11

Dankwoord (Acknowledgements)

Lijst van publicaties

Curriculum Vitae 


\section{DANKWOORD (ACKNOWLEDGEMENTS)}

Nog een paar pagina's en dan is 'het boekje' eindelijk af! Het mocht ook wel eens gebeuren. Graag wil ik ook nog een tweetal bladzijden spenderen aan het bedanken van een groot aantal mensen die elk op hun eigen manier aan het onderzoek en aan dit proefschrift bijgedragen hebben.

Allereerst mijn promotor, Professor van Kerrebroeck, bedankt voor de mogelijkheid om bij de afdeling Urologie in Maastricht te kunnen promoveren, voor de steun die u mij hierbij gegeven heeft en voor het in mij gestelde vertrouwen.

Professor de Bie, beste Rob, bedankt voor de hulp bij het methodische deel van het maken van de onderzoeksopzet en bij de statistische analyses. Ook al zijn door de jaren heen onze beider agenda's nóg voller geworden, ik kon altijd bij je terecht voor goede raad, niet noodzakelijkerwijs over statistiek. Bedankt daarvoor!

Beste Ernest Weil, je hebt me alle klinische in-en-outs van de sacrale neuromodulatie geleerd. Ook was er altijd ruimte voor advies en steun voor allerhande andere zaken. Hartelijk bedankt!

Professor Jaques van Lankveld en Nele Gielen, bedankt voor jullie bijdrage aan het sexuologie artikel (hoofdstuk 7).

Mijn collega-onderzoekers; Enrico Dassen, Rick de Jongh, Kelly Hoenjet, en Luba Drossaert, bedankt voor de steun, de gesprekken tijdens de lunch en het feit dat ik wanneer nodig eventjes kon ventileren! Hoewel mijn hoekje in ons 'Paleis van de Wetenschap' van pakweg 4 bij 2 meter niet altijd even netjes opgeruimd was, leverde dat nooit problemen op.

Miriam Dambros, at the beginning of your research in Maastricht we agreed that you would teach me Spanish in exchange for me teaching you Dutch. Although this deal has not really worked out, I would like to thank you sincerely for the company and the friendship during your stay in Maastricht. Thank you for choosing me as one of your paranimfs. Good luck introducing SNM in Brazil! Mijn voorganger Wout Scheepens en mijn opvolgers Dennis Oerlemans en Tom Marcelissen, bedankt voor jullie bijdragen aan dit proefschrift en voor het 'passen' op onze onderzoeks data.

Sacha Koch en Jarno Melenhorst, 'conculega's' van de afdeling Heelkunde. Bedankt voor de prettige samenwerking en de gezelligheid in en buiten het ziekenhuis. 
Ook een woord van dank aan de medische staf en arts-assistenten van de afdeling Urologie in het AZM, in het bijzonder aan dr. Gommert van Koeveringe. Bedankt voor de leerzame, boeiende en gezellige tijd die ik heb gehad tijdens mijn aanstelling als arts-onderzoeker Urologie.

De secretaresses van de afdeling Urologie en met name Marjon, Cindy en Anja. Bedankt voor jullie ondersteuning, luisterend oor en voor het belletje wanneer er weer eens vla(ai) getrakteerd werd.

Ook het personeel van de polikliniek en de functieafdeling Urologie, hartelijk bedankt voor de onnavolgbare manier waarop jullie je dagelijks werk uitvoeren. Altijd was er iets te regelen als er weer onverwacht een patiënt gezien, urodynamisch onderzocht of getest moest worden.

Revalidatieartsen, arts-assistenten en andere medewerkers in de verschillende revalidatie instellingen waar ik mijn ANIOS-schap en opleiding tot revalidatiearts heb mogen volgen, bedankt voor de prettige samenwerking, jullie belangstelling voor mijn 'ongoing project' en jullie aanmoedigingen. Dat geldt ook voor mijn huidige collega's en medewerkers in Via Reva, centrum voor Revalidatie in Apeldoorn. Op naar het volgende project!

Beste studievrienden/viendinnen, we begonnen als De Drie Musketiers en er kwamen er steeds meer bij. Bedankt voor de gezelligheid, ook gedurende de jaren dat wij in het 'verre' (het ligt er maar aan hoe je 't bekijkt) Maastricht woonden. Dan is een barbecue en een extra logeerkamer erg handig.

Irma, Johan en Martin, we doen alle vier totaal verschillende dingen. Toch is het altijd vertrouwd als we elkaar weer opzoeken. Nu Irma weer in Nederland is neergestreken en ik mijn promotie achter de rug heb, moeten we misschien toch weer eens de Van Voskuilen Band nieuw leven inblazen.

Mam, papa en jij hebben me altijd gestimuleerd om mijn eigen weg te gaan en me daarbij onvoorwaardelijk gesteund. Dank je wel!

Lieve Monique, dank je wel dat je er voor me bent. En voor jouw support voor wat dit proefschrift betreft, maar ook voor al het andere. Daarom nog een keer zwart op wit: ik hou van je!

Cas, Joost en Ruben, ik vind het fantastisch om jullie vader te zijn en me elke dag door jullie te laten verwonderen. Bedankt daarvoor! 


\section{OVER DE AUTEUR VAN DIT PROEFSCHRIFT}

Anco van Voskuilen werd op 24 februari 1974 in Ede geboren.

In 1994 haalde hij zijn VWO diploma aan het Veluws College in Apeldoorn. Direct daarna startte hij met de studie Geneeskunde aan de Radboud Universiteit in Nijmegen. In 2000 werd het artsexamen behaald.

In November 2002 begon hij op de afdeling Urologie in het Academisch Ziekenhis in Maastricht met het onderzoek wat uiteindelijk tot dit proefschrift leidde.

Tijdens een ANIOS-schap Neurologie in 2006 in het Rijnstate Ziekenhuis in Arnhem kwam hij in aanraking met de revalidatiegeneeskunde. Na een ANIOS-schap Revalidatiegeneeskunde in revalidatiecentrum Groot-Klimmendaal in Arnhem begon hij in maart 2007 met de opleiding tot revalidatiearts in respectievelijk de Sint Maartenskliniek, revalidatiecentrum de Tolbrug in 's Hertogenbosch en de afdeling Revalidatiegeneeskunde in het UMC St Radboud in Nijmegen.

In 2011 werd hij geregistreerd als revalidatiearts en momenteel werkt hij zowel in Revaldiatiecentrum Via Reva in Apeldoorn met als aandachtsgebied neurorevalidatie als in het Gelre Ziekenhuis, eveneens in Apeldoorn.

Hij is gehuwd met Monique en heeft drie zoons; Cas (5 jaar) en tweeling Joost en Ruben (3 jaar). 



\section{LIJST VAN PUBLICATIES}

van Voskuilen AC, Oerlemans DJ, Gielen N, Lansen-Koch SM, Weil EH, van Lankveld JJ, van den Hombergh U, Baeten CG, van Kerrebroeck PE. Sexual Response in Patients Treated with Sacral Neuromodulation for Lower Urinary Tract Symptoms or Fecal Incontinence. Urol Int. 2012 Apr 26.

Oerlemans DJ, van Voskuilen AC, Marcelissen T, Weil EH, de Bie RA, Van Kerrebroeck PE. Is on-demand sacral neuromodulation in patients with OAB syndrome a feasible therapy regime? Neurourol Urodyn. 2011 Nov;30(8):1493-6.

van Kerrebroeck PE, van Voskuilen AC, Heesakkers JP, Lycklama á Nijholt AA, Siegel S, Jonas U, Fowler CJ, Fall M, Gajewski JB, Hassouna MM, Cappellano F, Elhilali MM, Milam DF, Das AK, Dijkema HE, van den Hombergh U. Results of sacral neuromodulation therapy for urinary voiding dysfunction: outcomes of a prospective, worldwide clinicalstudy.

J Urol. 2007 Nov;178(5):2029-34.

Van Voskuilen AC, Oerlemans DJ, Weil EH, van den Hombergh U, van Kerrebroeck PE. Medium-term experience of sacral neuromodulation by tined lead implantation. BJU Int. 2007 Jan;99(1):107-10.

van Voskuilen AC, Oerlemans DJ, Weil EH, de Bie RA, van Kerrebroeck PE. Long term results of neuromodulation by sacral nerve stimulation for lower urinary tract symptoms: a retrospective single center study. EurUrol. 2006 Feb;49(2):366-72.

van Voskuilen AC, Weil EH, De Bie RA, van Kerrebroeck EV. Ten years' experience in neuromodulation in Maastricht. Neuromodulation. $2005 \mathrm{Jul} ; 8(3): 183-5$.

van Voskuilen AC, Weil EH, van Kerrebroeck EV. Tined lead implantation: results of the first 37 implants in Maastricht. Neuromodulation. $2005 \mathrm{Jul} ; 8(3): 182-3$.

Vos PE, van Voskuilen AC, Beems T, Krabbe PF, Vogels OJ.Evaluation of the traumatic coma data bank computed tomography classification for severe head injury. J Neurotrauma. 2001 Jul;18(7):649-55.

A.C. van Voskuilen, E.H.J. Weil, M. Dambros and Ph. E.V.A. van Kerrebroeck:

Neuromodulation: Clinical experiences with the new minimal invasive approach. www.urovirt.org.br, volume 8 (3), 2004. 
A.C. van Voskuilen, E.H.J. Weil, Ph.E.V. van Kerrenbroeck: Neuromodulatie: Klinische ervaringen met de nieuwe minimaal-invasieve methode.

BAU Newsletter sept. 20044 (3) blz. 20-24.

W.A. Scheepens, A.C. van Voskuilen, G.A. van Koeveringe, E.H.J. Weil, Ph.E.V van Kerrebroeck: Sacrale neuromodulatie: de indicatiestelling.

BAU Newsletter sept. 20044 (3) blz. 25-34. 